\title{
HIGH-FREQUENCY BACKREACTION FOR THE EINSTEIN EQUATIONS UNDER POLARIZED $\mathbb{U}(1)$ SYMMETRY
}

\author{
CÉCILE HUNEAU AND JONATHAN LUK
}

\begin{abstract}
Known examples in plane symmetry or Gowdy symmetry show that given a 1-parameter family of solutions to the vacuum Einstein equations, it may have a weak limit which does not satisfy the vacuum equations, but instead has a non-trivial stress-energy-momentum tensor. We consider this phenomenon under polarized $\mathbb{U}(1)$ symmetry - a much weaker symmetry than most of the known examples - such that the stress-energy-momentum tensor can be identified with that of multiple families of null dust propagating in distinct directions. We prove that any generic local-in-time small-data polarized-U(1)-symmetric solution to the Einstein-multiple null dust system can be achieved as a weak limit of vacuum solutions. Our construction allows the number of families to be arbitrarily large, and appears to be the first construction of such examples with more than two families.
\end{abstract}

\section{INTRODUCTION}

There is a long tradition in the physics literature since the work of Isaacson 8, 9 , studying high frequency backreaction in general relativity [2, 4, 5, 14. In particular, it has been observed that suitably scaled smallamplitude but high-frequency gravitational waves give rise to a non-trivial " $O(1)$ " contribution to the background metric which mimics an "effective matter field". For the purpose of this paper, we consider the following mathematical formulation by Burnett [1]: On a fixed manifold, consider a one-parameter family of Lorentzian metrics $g_{\lambda}$ for $\lambda \in\left(0, \lambda_{0}\right], \lambda_{0} \in \mathbb{R}$ which satisfies the Einstein vacuum equation] 1

$$
R_{\mu \nu}\left(g_{\lambda}\right)=0 .
$$

Assume moreover that there exists a metric $g_{0}$ such that as $\lambda \rightarrow 0$,

- $g_{\lambda}$ converges to $g_{0}$ uniformly on compact sets; and

- the derivatives of $g_{\lambda}$ converges weakly in $L^{2}$ to the derivatives of $g_{0}$.

Due to the nonlinearity of the equations, it can happen that the limiting metric $g_{0}$ is a solution to the Einstein equations which is not vacuum, but has a non-trivial stress-energy-momentum tensor, i.e.,

$$
R_{\mu \nu}\left(g_{0}\right)-\frac{1}{2}\left(g_{0}\right)_{\mu \nu} R\left(g_{0}\right)=T_{\mu \nu}
$$

for some $T_{\mu \nu} \neq 0$. We will call the tensor $T_{\mu \nu}$ for the limiting metric the effective stress-energy-momentum tensor.

Two questions arise in this context: First, what are the possible matter model: 2 that can arise as limiting effective stress-energy-momentum tensor? Second, if a certain matter model arise as such limits, is it true that all solutions (at least in a certain solution regime) to the Einstein equations coupled with that matter model can be achieved as a limit of vacuum solutions in the sense described above?

In connection to the questions above, Burnett [1] conjectured that all such limits can be identified as solutions to the Einstein-massless Vlasov system. Moreover, in the same work, he also asked the question whether all solutions to the Einstein-massless Vlasov system can be achieved as a limit (in the sense described above) of solutions to the Einstein vacuum equations. Both of these questions remain open. In the direction of constraining possible effective stress-energy-momentum tensors, it is known by the work of Green-Wald [4 that the effective stress-energy-momentum tensor $T_{\mu \nu}$ verifies non-trivial constraints: in fact, it must be traceless and satisfy the weak energy condition. These conditions are consistent with - but clearly much weaker than - requiring $T_{\mu \nu}$ be identifiable as that of massless Vlasov matter. In the direction of constructing examples of effective stress-energy-momentum tensors, in the only known examples in the

\footnotetext{
${ }^{1}$ We use the notation that for a given metric $g, R_{\mu \nu}$ denotes its Ricci tensor while $R(g)$ denotes its scalar curvature.

${ }^{2}$ There are important caveats for this question though: $T_{\mu \nu}$ that arises may not always be identifiable with some well-known matter models. Also, in general for a given $T_{\mu \nu}$, there is no reason to expect that it corresponds uniquely to some matter models.
} 
literature, $T_{\mu \nu}$ of the limiting spacetime can be identified with that of one or two families of null dust. This can indeed be viewed at least formally as a special (singular) case of massless Vlasov matter.

The aim of this paper is to construct one-parameter families of metrics such that the effective stressenergy-momentum tensor represents that of a sum of an arbitrary finite number of families of null dust propagating in different directions. In particular, our work can be viewed as a first step towards approaching the questions of Burnett: one may hope to eventually construct more general solutions to the Einsteinmassless Vlasov system by taking the number of families of null dust to infinity. Our construction assumes that the one-parameter families of metrics and the limiting metric are polarized $\mathbb{U}(1)$ symmetric. In this setting, not only do we construct particular examples, we show that in fact any generic, polarized $\mathbb{U}(1)$ symmetric, small data solution to the Einstein-null dust system (with an arbitrary finite number of families of null dust) arise as a weak limit of solutions to the Einstein vacuum equations.

The mathematical challenge in studying this problem is that the metrics one construct necessarily oscillate with high frequency. In the process, one needs to handle solutions with very weak uniform estimates and deal with issues familiar in the setting of low-regularity problems. In fact, in order to obtain a non-trivial stress-energy-momentum tensor in the limit, the one parameter family of metrics can at best obey uniform $W^{1, \infty}$ estimates, but not any uniform estimates for the higher order derivatives. On the other hand, even to ensure uniform time of existence of solutions, one in general needs the metrics to be in $W^{2,2}$ uniformly, cf. 10. Most previous examples [1, 5, rely on the fact that under plane or polarized Gowdy symmetry, the solutions to the Einstein vacuum equations, even when the regularity is low, are explicit. On the other hand, these symmetries limits the effective stress-energy-momentum tensor to represent at most two families of null dusts.

One way to obtain a low regularity local existence result for the Einstein equations which is consistent with the high frequency oscillations described above is to assume that the initial data are more regular in some directions. This has been achieved in the recent work of Luk-Rodnianski [11, 12], which constructed via solving a characteristic initial problem a class of low-regularity solutions to the Einstein vacuum equation on $S \times \mathbb{R}^{2}$ (where $S$ is a 2-dimensional surface) with no exact symmetries such that all tangential derivatives of the metric along $S$ are regular, while general derivatives of the metric are only in $L^{2}$. With such an existence result, one can construct a sequence of metrics which in the limit give rise to an effective stressenergy-momentum tensor of two families of null dust, with no symmetry assumptions; see the forthcoming [13. Nevertheless, even in this construction, since the metric is regular along $S$, the oscillations in the metrics are limited to 2 dimensions and therefore the effective stress-energy-momentum tensor can represent at most two families of null dust.

In contrast, our present work gives the first result such that the metrics $g_{\lambda}$ are allowed to oscillate in more than 2 dimensions. As a consequence, we obtain stress-energy-momentum tensors representing an arbitrary number of families of null dust. To obtain our result, we do not attempt to prove general existence results which are consistent with the metric oscillating in more than 2 dimensions. Instead, our analysis is specific to the problem at hand, and we construct a parametrix to capture the high frequency oscillations of the metric. Moreover, we rely heavily on the structure of the Einstein equations under polarized $\mathbb{U}(1)$ symmetry. We refer the reader Section 1.2 for further discussions on the ideas of the proof, after we give an informal discussion of the main result of the paper in the next subsection.

1.1. Main result. To construct our examples, we will work under polarized $\mathbb{U}(1)$ symmetry ${ }^{4}$, which gives certain important technical simplifications that we will explain later. In addition to merely constructing examples, we will in fact show that under polarized $\mathbb{U}(1)$ symmetry, up to some technical conditions, $\underline{\text { most }}$ sufficient small and sufficient regular local solutions $g_{0}$ to (1.2) with $T_{\mu \nu}$ given by a sum of a finite number of families of null dust can be weakly approximated by vacuum solutions $g_{\lambda}$ to (1.1) in the sense that we described above.

We now further describe our setting: We study solutions of the vacuum Einstein equations of the form $\left(I \times \mathbb{R}^{3}{ }^{(4)} g\right)$, where $I \subset \mathbb{R}$ is an interval, with

$$
{ }^{(4)} g=e^{-2 \phi} g+e^{2 \phi}\left(d x^{3}\right)^{2},
$$

\footnotetext{
${ }^{3}$ The original motivation for such a low-regularity result in [11 12 is to study the propagation and interaction of impulsive gravitational waves (solutions such that the Riemann curvature tensor has a delta singularity on an embedded null hypersurface).

${ }^{4}$ Notice that in contrast to plane symmetry or Gowdy symmetry which we discussed above, the symmetry group $\mathbb{U}(1)$ is only 1-dimensional.
} 
wher $5: I \times \mathbb{R}^{2} \rightarrow \mathbb{R}$ is a scalar function and $g$ is a Lorentzian metric on $I \times \mathbb{R}^{2}$. The vector field $\partial_{x_{3}}$ is Killing and hypersurface orthogonal. Then Einstein vacuum equations $R\left({ }^{(4)} g\right)_{\mu \nu}=0$ are equivalent to the following system for $(g, \phi)$ :

$$
\left\{\begin{array}{l}
\square_{g} \phi=0 \\
R_{\mu \nu}(g)=2 \partial_{\mu} \phi \partial_{\nu} \phi
\end{array}\right.
$$

As mentioned above, we want to show that the weak limits of vacuum solutions (i.e., solutions to (1.3)) give rise to solutions with a stress-energy-momentum tensor which represents a sum of $N$ families of null dust, traveling in arbitrary directions (as long as the directions associated to each pair of families are "angularly separated", see Section 4.1). More precisely, we consider a quadruple $\left(g, \phi, F_{\mathbf{A}}, u_{\mathbf{A}}\right)$, with $\mathbf{A} \in \mathcal{A}$ for some finite set $\mathcal{A}$ with $|\mathcal{A}|=N$, where $g$ is a Lorentzian metric on $I \times \mathbb{R}^{2}, \phi: I \times \mathbb{R}^{2} \rightarrow \mathbb{R}$ is a scalar function, $F_{\mathbf{A}}: I \times \mathbb{R}^{2} \rightarrow \mathbb{R}_{\geq 0}$ is the density of the null dust for each $\mathbf{A}$ and $u_{\mathbf{A}}: I \times \mathbb{R}^{2} \rightarrow \mathbb{R}$ is an eikonal function such that $\left(d u_{\mathbf{A}}\right)^{\sharp}$ is the direction of propagation of the null dust for each $\mathbf{A}$, which is a solution to the system

$$
\left\{\begin{array}{l}
R_{\mu \nu}(g)=2 \partial_{\mu} \phi \partial_{\nu} \phi+\sum_{\mathbf{A}}\left(F_{\mathbf{A}}\right)^{2} \partial_{\mu} u_{\mathbf{A}} \partial_{\nu} u_{\mathbf{A}}, \\
\square_{g} \phi=0, \\
2\left(g^{-1}\right)^{\alpha \beta} \partial_{\alpha} u_{\mathbf{A}} \partial_{\beta} F_{\mathbf{A}}+\left(\square_{g} u_{\mathbf{A}}\right) F_{\mathbf{A}}=0, \\
\left(g^{-1}\right)^{\alpha \beta} \partial_{\alpha} u_{\mathbf{A}} \partial_{\beta} u_{\mathbf{A}}=0 .
\end{array}\right.
$$

Notice that the system (1.4) corresponds to the following system 6 in the original $(3+1)$-dimensional spacetime $I \times \mathbb{R}^{3}$ :

$$
\left\{\begin{array}{l}
R_{\mu \nu}\left({ }^{(4)} g\right)=\sum_{\mathbf{A}}\left(F_{\mathbf{A}}\right)^{2} \partial_{\mu} u_{\mathbf{A}} \partial_{\nu} u_{\mathbf{A}}, \\
2\left({ }^{(4)} g^{-1}\right)^{\alpha \beta} \partial_{\alpha} u_{\mathbf{A}} \partial_{\beta} F_{\mathbf{A}}+\left(\square_{(4)} u_{\mathbf{A}}\right) F_{\mathbf{A}}=0, \\
\left({ }^{(4)} g^{-1}\right)^{\alpha \beta} \partial_{\alpha} u_{\mathbf{A}} \partial_{\beta} u_{\mathbf{A}}=0 .
\end{array}\right.
$$

In other words, (1.4) indeed corresponds to the Einstein-null dust system with $N$ families of null dust.

Our main theorem can be stated informally as follows (and we refer the readers to Theorem 4.2 for a precise statement):

Theorem 1.1. Let $\left(g_{0}, \phi_{0}, F_{\mathbf{A}}, u_{\mathbf{A}}\right)$ be a sufficiently small and sufficiently regular local-in-time asymptotically conic solution to (1.4) such that

- The initial hypersurface is maximal;

- The $u_{\mathbf{A}}$ 's are angularly separated;

- A genericity condition holds.

Then $\left(g_{0}, \phi_{0}\right)$ can be weakly approximated by a 1-parameter family of solutions $\left(g_{\lambda}, \phi_{\lambda}\right)$ for $\lambda \in\left(0, \lambda_{0}\right)$, $\lambda_{0} \in \mathbb{R}$ to (1.3), i.e., in a suitable coordinate system, as $\lambda \rightarrow 0,\left(g_{\lambda}, \phi_{\lambda}\right) \rightarrow\left(g_{0}, \phi_{0}\right)$ uniformly on compact sets and the derivatives $\left(\partial g_{\lambda}, \partial \phi_{\lambda}\right) \rightarrow\left(\partial g_{0}, \partial \phi_{0}\right)$ weakly in $L^{2}$ (for each component).

It is not unlikely that the methods introduced in this paper can also be applied to the situation where the spacetime is only assumed to be $\mathbb{U}(1)$ symmetric without polarization. In that case, the Einstein vacuum equations no longer reduce to a system of Einstein-scalar wave equations, but instead reduce to a system of Einstein-wave map equations. The wave map equations are more nonlinear, but the semilinear terms satisfy the classical null condition, which therefore give hope to extending the results in the present paper. However, the full $(3+1)$-dimensional situation, even when restricted to "almost- $\mathbb{U}(1)$-symmetry", seems to require ideas beyond those introduced here.

1.2. Ideas of the proof. The main challenge in constructing our examples is that in order for backreaction to occur, $\phi_{\lambda}$ must oscillate in a manner that only their first derivatives can be assumed to be uniformly bounded and that their second derivatives (or in fact any " $1+\varepsilon$ derivative") must blow up as $\lambda \rightarrow 0$. This is much rougher than the required regularity under which the Einstein equations are known to be locally well-posed (see [10]). In [13, this issue was dealt with exactly with an improved local well-posedness result [12], devised first to understand impulsive gravitational waves, in the general setting where $\mathcal{M}=S \times \mathbb{R}^{2}$ and that the $S$-tangent derivatives of the metric are more regular.

In this paper, however, we take another route. Instead of proving local well-posedness result in general function spaces which $\left(g_{\lambda}, \phi_{\lambda}\right)$ belong, we exploit the fact that the $\left(g_{\lambda}, \phi_{\lambda}\right)$ are constructed to (weakly)

\footnotetext{
${ }^{5}$ Abusing notation slightly, we will also view $\phi$ as a function $\phi: I \times \mathbb{R}^{3} \rightarrow \mathbb{R}$ and $g$ as a metric on $I \times \mathbb{R}^{3}$, which do not depend on the variable $x^{3}$.

${ }^{6}$ To see this, simply note that (1) with the ansatz of the metric, and $\square_{g} \phi=0$, we have for $\alpha, \beta=0,1,2, R_{\alpha \beta}\left({ }^{(4)} g\right)=$ $R_{\alpha \beta}(g)-2 \partial_{\alpha} \phi \partial_{\beta} \phi, R_{3 \alpha}=R_{33}=0 ;(2) \square_{g}=\square_{(4) g}$ and (3) $u_{\mathbf{A}}, F_{\mathbf{A}}$ are independent of $x^{3}$. For these formulae, the reader can consult [3] Appendix VII].
} 
approximate $\left(g_{0}, \phi_{0}\right)$ and rely more on the regularity of $\left(g_{0}, \phi_{0}\right)$. In the proof, we construct a parametrix of the high frequency part of $\left(g_{\lambda}, \phi_{\lambda}\right)$ and prove that it is indeed a good approximation of the actual solution. In particular, we show that the "high frequency waves" in $\phi_{\lambda}$ approximately travel along characteristic hypersurfaces of the background metric $g_{0}$. As a consequence of our approach, we also obtain a precise

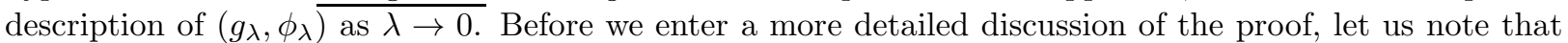
in order to carry out this analysis, we rely on the following three facts specific to $\mathbb{U}(1)$ symmetry:

(1) The Einstein vacuum equations reduce under the symmetry assumptions to the Einstein-scalar field system. Under this reduction, it is only the scalar field that contributes to the backreaction. In particular, the "high-frequency wave part" and the "geometry part" of the solution "separate".

(2) By introducing an elliptic gauge, the metric of the reduced system can be completely recovered by elliptic equations (i.e., the "geometry part" of the solution is more regular).

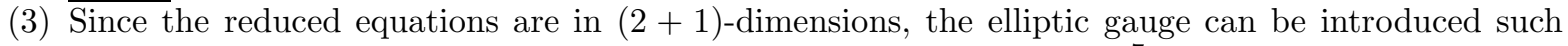
that the equations for the metric of the reduced system are semilinear 7 .

1.2.1. Model problem and first construction of parametrix. Working in an elliptic gauge, and thinking of $\mathfrak{g}$ as a metric component, the equations can roughly be modeled by the following model problem:

$$
\left\{\begin{array}{l}
\square_{g(\mathfrak{g})} \phi=0 \\
\Delta \mathfrak{g}=(\partial \phi)^{2}
\end{array}\right.
$$

where $g$ is a Lorentzian metric and $\Delta$ is the Laplacian for flat $\mathbb{R}^{2}$. This formulation captures the advantages of $\mathbb{U}(1)$ symmetry as described earlier, in that the "dynamical" part and the "geometric" part "separate", and that the geometric part satisfies a semilinear elliptic system.

We will construct our desired one-parameter family of solutions to (1.3) in the form

$$
\phi_{\lambda}=\phi_{0}+\sum_{\mathbf{A}} \lambda F_{\mathbf{A}} \cos \left(\frac{u_{\mathbf{A}}}{\lambda}\right)+\widetilde{\phi}_{\lambda}, \quad \mathfrak{g}_{\lambda}=\mathfrak{g}_{0}+\widetilde{\mathfrak{g}}_{\lambda}
$$

such that $\left(\partial \widetilde{\phi}_{\lambda}, \partial \widetilde{\mathfrak{g}}_{\lambda}\right) \rightarrow(0,0)$ uniformly on compact sets as $\lambda \rightarrow 0$. First, by virtue of the fact that $u_{\mathbf{A}}$ satisfies the eikonal equation for the background metric, and that $F_{\mathbf{A}}$ satisfies the transport equation in (1.4), $\sum_{\mathbf{A}} \lambda F_{\mathbf{A}} \cos \left(\frac{u_{\mathbf{A}}}{\lambda}\right)$ can be viewed as an approximate solution to the wave equation, since $\square_{g}\left(\sum_{\mathbf{A}} \lambda F_{\mathbf{A}} \cos \left(\frac{u_{\mathbf{A}}}{\lambda}\right)\right)$ consists of terms either of size $O(\lambda)$ or bounded in terms of $\widetilde{\mathfrak{g}}_{\lambda}$. Moreover,

$$
2 \partial_{\mu} \phi_{\lambda} \partial_{\nu} \phi_{\lambda} \rightarrow 2 \partial_{\mu} \phi_{0} \partial_{\nu} \phi_{0}+\sum_{\mathbf{A}}\left(F_{\mathbf{A}}\right)^{2} \partial_{\mu} u_{\mathbf{A}} \partial_{\nu} u_{\mathbf{A}}
$$

weakly in $L^{2}$ (with uniform $L^{\infty}$ bounds) which is exactly the form of the stress-energy-momentum tensor in (1.4).

However, in order for this parametrix to be useful, we at the very least need to say that $\widetilde{\phi}_{\lambda}$ is better than the main term $\sum_{\mathbf{A}} \lambda F_{\mathbf{A}} \cos \left(\frac{u_{\mathbf{A}}}{\lambda}\right)$. Suppose, say, by an expansion in $\lambda$, we hope to obtain that $\widetilde{\phi}_{\lambda}=O\left(\lambda^{2}\right)$, with a loss of $\lambda^{-1}$ for every derivative. Plugging this into (1.5), we have on the RHS of the elliptic equation a term

$$
\partial \widetilde{\phi}_{\lambda} \partial\left(\lambda F_{\mathbf{A}} \cos \left(\frac{u_{\mathbf{A}}}{\lambda}\right)\right)=O(\lambda)
$$

so that standard elliptic estimates give

$$
\left\|\widetilde{\mathfrak{g}}_{\lambda}\right\|_{W^{1, \infty}} \leq \lambda .
$$

Now we plug this back into the wave equation $\square_{g} \widetilde{\phi}_{\lambda}$ (for which $\widetilde{g}_{\lambda}$ enters through $\square_{g}$ ). When estimating the $H^{1}$ norm of $\widetilde{\phi}_{\lambda}$ using energy estimates, we need to control (in $L^{2}$ ) a term of the form

$$
\frac{1}{\lambda}\left(g_{\lambda}^{\alpha \beta}-g_{0}^{\alpha \beta}\right) \partial_{\alpha} u_{\mathbf{A}} \partial_{\beta} u_{\mathbf{A}} \cos \left(\frac{u_{\mathbf{A}}}{\lambda}\right) \text {. }
$$

However, with (1.8), this term is only $O(1)$ (instead of $O(\lambda)$ ) and the estimates cannot be closed. In order to deal with this, we need a more precise parametrix, which in particular captures the fact the $O(\lambda)$ term in $\widetilde{\phi}_{\lambda}$ are of high frequency, and moreover, that one can gain a smallness parameter from these high frequency terms for appropriate inversions of $\Delta$ and $\square_{g}$.

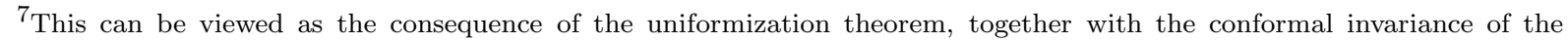
2-dimensional Laplacian.
} 
1.2.2. Well-preparedness of the eikonal functions and a more precise construction of the parametrix. The more precise parametrix for $\phi_{\lambda}$ takes the form (for details see Section 4.4)

$$
\begin{aligned}
\phi_{\lambda}= & \phi_{0}+\sum_{\mathbf{A}} \lambda F_{\mathbf{A}} \cos \left(\frac{u_{\mathbf{A}}}{\lambda}\right)+\sum_{\mathbf{A}} \lambda^{2} \widetilde{F}_{\mathbf{A}} \sin \left(\frac{u_{\mathbf{A}}}{\lambda}\right) \\
& +\sum_{\mathbf{A}} \lambda^{2} \widetilde{F}_{\mathbf{A}}^{(2)} \cos \left(\frac{2 u_{\mathbf{A}}}{\lambda}\right)+\sum_{\mathbf{A}} \lambda^{2} \widetilde{F}_{\mathbf{A}}^{(3)} \sin \left(\frac{3 u_{\mathbf{A}}}{\lambda}\right)+\mathcal{E}_{\lambda},
\end{aligned}
$$

where one should think of $\widetilde{F}_{\mathbf{A}}, \widetilde{F}_{\mathbf{A}}^{(2)}$ and $\widetilde{F}_{\mathbf{A}}^{(3)}$ as bounded and $\mathcal{E}_{\lambda}$ as a smaller remainder, bounded in $H^{1}$ by $\lambda^{2}$. We also decompose the metric components accordingly:

$$
\mathfrak{g}=\mathfrak{g}_{0}+\mathfrak{g}_{1}+\mathfrak{g}_{2}+\mathfrak{g}_{3}
$$

so that roughly speaking $\mathfrak{g}_{1}$ are $O\left(\lambda^{2}\right)$ (high-frequency) terms and $\mathfrak{g}_{2}$ are $O\left(\lambda^{3}\right)$ (high-frequency) terms.

In order to control the parametrix up to this order, notice that certain terms (in particular $\widetilde{F}_{\mathbf{A}}, \mathcal{E}_{\lambda}, \mathfrak{g}_{2}$ and $\mathfrak{g}_{3}$; see Section 4.4) couple. As a consequence, one needs to carefully handle the regularity of these terms (in addition to estimating their sizes in terms of $\lambda$ ).

In the parametrix for $\phi_{\lambda}$, we only explicitly keep track of the terms oscillating in null directions (with phase function proportional to $u_{\mathbf{A}}$ ). This construction is based on the facts that

- Any high frequency term must be highly oscillatory in the spatial direction, and one gains $\lambda^{2}$ upon inverting $\Delta$;

- Any high frequency term arising from non-parallel interaction (i.e., from $u_{\mathbf{A}}$ and $u_{\mathbf{B}}$ with $\mathbf{A} \neq \mathbf{B}$ ) can be treated "elliptically" for the wave part, and inverting $\square_{g}$ gives a smallness of $\lambda^{2}$.

To achieve these, we need to use the assumption that $\left\{u_{\mathbf{A}}\right\}_{\mathbf{A} \in \mathcal{A}}$ is angularly separated (see Definition 4.1) and also to exploit the symmetry $\left(u_{\mathbf{A}}, F_{\mathbf{A}}\right) \mapsto\left(c_{\mathbf{A}} u_{\mathbf{A}}, c_{\mathbf{A}}^{-1} F_{\mathbf{A}}\right)$ to prepare the eikonal functions well (see Section 4.2).

There are also additional useful structures hidden in the expression of the parametrix which we exploit. For instance, when controlling terms as in (1.7), we use that the interaction of the $F$ term with any of $\left(\widetilde{F}, \widetilde{F}^{(2)}, \widetilde{F}^{(3)}\right)$ is necessarily high frequency, and one can indeed gain powers of $\lambda$.

1.2.3. Handling the $\partial_{t}$ derivatives of the error of the metric. One important challenge we face is that the $\partial_{t}$ derivative of the metric obeys worse estimates (compared to the spatial derivatives). This is because the metric components solve elliptic equations on a spatial slice, and to control their $\partial_{t}$ derivative requires differentiating the equation. Because of similar reasons, and also the fact that $\widetilde{F}_{\mathbf{A}}$ couples with $\mathfrak{g}_{3}$, the second time derivative $\partial_{t}^{2} \widetilde{F}_{\mathbf{A}}$ also obey worse estimates compared to when at least one of the derivatives is a spatial derivatives.

Fortunately, one can still handle the situation with the worse estimates using the structure of the Einstein equations! We highlight a few points below: Note that in particular, except for point (3), we strongly use the fact that we are solving the Einstein equations and the structure that we rely on is not captured by the model problem (1.5).

(1) Hidden in the evolution is the propagation of the maximality of the hypersurfaces. This allows us to rewrite the $\partial_{t}$ derivative of a metric component (more precisely, $\gamma$, to be introduced in (3.5)) in terms of spatial derivatives of (precisely, $\beta$ ). Hence, such a term is better than expected.

(2) Importantly, using in particular the above observation, one shows that the two uncontrollable $\partial_{t^{-}}$ derivative error terms, one involving $\partial_{t} \mathfrak{g}_{3}$ and one involving $\partial_{t}^{2} \widetilde{F}_{\mathbf{A}}$, cancel; see Proposition 6.12

(3) Another type of error terms is of the form $\partial_{t} \mathfrak{g}_{3}$ multiplied by a low frequency term. Here, one can exploit the low frequency term using an integration by parts argument; see Proposition 6.21.

1.3. Outline of the paper. The remainder of the paper will be structured as follows:

- In Section 2, we introduce the notations and functions spaces that we will use.

- In Section 3, we give a precise definition of our gauge condition, and state the local well-posedness results for (1.3) and (1.4) from our companion paper [7.

- In Section 4, we give a precise statement of the main theorem (cf. Theorems 1.1 and 4.2). We also discuss preliminary steps of the proof: rescaling of the eikonal functions, construction of initial data to the one-parameter family of solutions, and construction of the parametrix for $\phi_{\lambda}$ and $g_{\lambda}$.

- In next few sections are devoted to the proof of the main theorem: 
- The proof proceeds by a bootstrap argument, and the main setup and bootstrap assumptions are given in Section 5 .

- In Section 6, we derive the estimates for the scalar field.

- In Section 7, we derive the estimates for the metric components.

- Finally, in Section 8, we conclude the proof.

- In Appendix \$ we collect some results about Sobolev embedding, product estimates and elliptic estimates in weighted Sobolev spaces in $\mathbb{R}^{2}$.

Acknowledgements. J. Luk thanks Igor Rodnianski and Robert Wald for stimulating discussions. Most of this work was carried out when both authors were in Cambridge University. C. Huneau is supported by the ANR-16-CE40-0012-01. J. Luk is supported in part by a Terman fellowship.

\section{Notations And FUnCtion SPACES}

Ambient space and coordinates In this paper, we will be working on the ambient manifold $\mathcal{M}:=$ $I \times \mathbb{R}^{2}$, where $I \subset \mathbb{R}$ is an interval. The space will be equipped with a system of coordinates $\left(t, x^{1}, x^{2}\right)$. We will use $x^{i}$ with the lower case Latin index $i, j=1,2$ and will also sometime denote $x^{0}=t$.

Conventions with indices We will use the following conventions:

- Lower case Latin indices run through the spatial indices 1,2 , while lower case Greek indices run through all the spacetime indices.

- Repeat indices are always summed over: where lower case Latin indices sum over the spatial indices 1,2 and lower case Greek indices sum over all indices $0,1,2$.

- Unless otherwise stated, lower case Latin indices are always raised and lowered with respect to the standard Euclidean metric $\delta_{i j}$.

- In contrast, lower case Greek indices are raised and lowered with respect to the spacetime metric $g$. In cases where there are more than one spacetime metric in the immediate context, we will not use this convention but will instead spell out explicitly how indices are raised and lowered.

Differential operators We will use the following conventions for differential operators:

- $\partial$ denotes partial derivatives in the coordinate system $\left(t, x^{1}, x^{2}\right)$. We will frequently write $\partial_{i}$ for $\partial_{x^{i}}$. In particular, we denote

$$
|\partial \xi|^{2}=\left(\partial_{t} \xi\right)^{2}+\sum_{i=1}^{2}\left(\partial_{x^{i}} \xi\right)^{2} .
$$

- The above $\partial$ notation also applied to rank-r covariant tensors $\xi_{\mu_{1} \ldots \mu_{r}}$ tangential to $I \times \mathbb{R}^{2}$ to mean

$$
|\partial \xi|^{2}=\sum_{\mu_{1}, \ldots, \mu_{r}=t, x^{1}, x^{2}}\left|\partial \xi_{\mu_{1} \ldots \mu_{r}}\right|^{2}
$$

and to rank- $r$ covariant tensors $\xi_{i_{1} \cdots_{r}}$ tangential to $\mathbb{R}^{2}$ to mean

$$
|\partial \xi|^{2}=\sum_{i_{1} \ldots i_{r}=x^{1}, x^{2}}\left|\partial \xi_{i_{1} \ldots i_{r}}\right|^{2} .
$$

- $\Delta$ and $\nabla$ denotes the spatial Laplacian and the spatial gradient on $\mathbb{R}^{2}$ with the standard Euclidean metric. In particular, we use the convention

$$
|\nabla \xi|^{2}=\sum_{i=1}^{2}\left|\partial_{x^{i}} \xi\right|^{2}
$$

- $D$ denotes the Levi-Civita connection associated to the spacetime metric $g$.

- $\square_{g}$ denotes the Laplace-Beltrami operator on functions, i.e.,

$$
\square_{g} \xi:=\frac{1}{\sqrt{|\operatorname{det} g|}} \partial_{\mu}\left(\left(g^{-1}\right)^{\mu \nu} \sqrt{|\operatorname{det} g|} \partial_{\nu} \xi\right) .
$$

- $\mathcal{L}$ denotes the Lie derivatives.

- $e_{0}$ defines the vector field $e_{0}=\partial_{t}-\beta^{i} \partial_{x^{i}}$ (where $\beta$ will be introduced in (3.5)). We will often use the differential operator $\mathcal{L}_{e_{0}}$.

- $L$ denotes the Euclidean conformal Killing operator acting on vectors on $\mathbb{R}^{2}$ to give a symmetric traceless (with respect to $\delta$ ) covariant 2-tensor, i.e.,

$$
(L \xi)_{i j}:=\delta_{j \ell} \partial_{i} \xi^{\ell}+\delta_{i \ell} \partial_{j} \xi^{\ell}-\delta_{i j} \partial_{k} \xi^{k}
$$


Functions spaces We will work with standard function spaces $L^{p}, H^{k}, C^{m}, C_{c}^{\infty}$, etc. and assume the standard definitions. The following conventions will be important:

- Unless otherwise stated, all function spaces will be taken on $\mathbb{R}^{2}$ and the measures will be taken to be the 2D Lebesgue measure $d x$.

- When applied to quantities defined on a spacetime $I \times \mathbb{R}^{2}$, the norms $L^{p}, H^{k}, C^{m}$ denote fixed-time norms (unless otherwise stated). In particular, if in an estimate the time $t \in I$ in question is not explicitly stated, then it means that the estimate holds for all $t \in I$ for the time interval $I$ that is appropriate for the context.

We will also work in weighted Sobolev spaces, which are well-suited to elliptic equations. We recall here the definition, together with the definition of weighted Hölder space. The properties of these spaces that we need are listed in Appendix $\mathrm{A}$.

Definition 2.1. Let $m \in \mathbb{N}, 1 \leq p<\infty, \delta \in \mathbb{R}$. The weighted Sobolev space $W_{\delta, p}^{m}$ is the completion of $C_{0}^{\infty}$ under the norm

$$
\|u\|_{W_{\delta, p}^{m}}=\sum_{|\beta| \leq m}\left\|\left(1+|x|^{2}\right)^{\frac{\delta+|\beta|}{2}} \nabla^{\beta} u\right\|_{L^{p}}
$$

We will use the notation $H_{\delta}^{m}=W_{\delta, 2}^{m}$ and $L_{\delta}^{p}=W_{\delta, p}^{0}$.

The weighted Hölder space $C_{\delta}^{m}$ is the complete space of m-times continuously differentiable functions under the norm

$$
\|u\|_{C_{\delta}^{m}}=\sum_{|\beta| \leq m}\left\|\left(1+|x|^{2}\right)^{\frac{\delta+|\beta|}{2}} \nabla^{\beta} u\right\|_{L^{\infty}}
$$

Finally, let us introduce the convention that we will use the above function spaces for both tensors and scalars on $\mathbb{R}^{2}$, where the norms in the case of tensors are understood componentwise.

\section{The elliptic gauge and local Well-posedness for (1.3) and (1.4)}

3.1. Elliptic gauge. We write the $(2+1)$-dimensional metric $g$ on $\mathcal{M}:=I \times \mathbb{R}^{2}$ in the form

$$
g=-N^{2} d t^{2}+\bar{g}_{i j}\left(d x^{i}+\beta^{i} d t\right)\left(d x^{j}+\beta^{j} d t\right) .
$$

Let $\Sigma_{t}:=\left\{\left(s, x^{1}, x^{2}\right): s=t\right\}$ and $e_{0}=\partial_{t}-\beta^{i} \partial_{i}$, which is a future directed normal to $\Sigma_{t}$. We introduce the second fundamental form of the embedding $\Sigma_{t} \subset \mathcal{M}$

$$
K_{i j}=-\frac{1}{2 N} \mathcal{L}_{e_{0}} \bar{g}_{i j}
$$

We decompose $K$ into its trace and traceless parts.

$$
K_{i j}=: H_{i j}+\frac{1}{2} \bar{g}_{i j} \tau \text {. }
$$

Here, $\tau:=\operatorname{tr}_{\bar{g}} K$ and $H_{i j}$ is therefore traceless with respect to $\bar{g}$.

Introduce the following gauge conditions:

- $\bar{g}$ is conformally flat, i.e., for some function $\gamma$,

$$
\bar{g}_{i j}=e^{2 \gamma} \delta_{i j}
$$

- The constant $t$-hypersurfaces $\Sigma_{t}$ are maximal

$$
\tau=0 .
$$

By (3.1), it follows that

$$
g=-N^{2} d t^{2}+e^{2 \gamma} \delta_{i j}\left(d x^{i}+\beta^{i} d t\right)\left(d x^{j}+\beta^{j} d t\right) .
$$

Hence the determinant of $g$ is given by

$$
\operatorname{det}(g)=e^{2 \gamma} \beta^{2}\left(-e^{4 \gamma} \beta^{2}\right)+e^{2 \gamma}\left(e^{2 \gamma}\left(-N^{2}+e^{2 \gamma}|\beta|^{2}\right)-e^{4 \gamma} \beta^{1} \beta^{1}\right)=-e^{4 \gamma} N^{2} .
$$

Moreover, the inverse $g^{-1}$ is given by

$$
g^{-1}=\frac{1}{N^{2}}\left(\begin{array}{ccc}
-1 & \beta^{1} & \beta^{2} \\
\beta^{1} & N^{2} e^{-2 \gamma}-\beta^{1} \beta^{1} & -\beta^{1} \beta^{2} \\
\beta^{2} & -\beta^{1} \beta^{2} & N^{2} e^{-2 \gamma}-\beta^{2} \beta^{2}
\end{array}\right) .
$$


In this gauge, the first equation in (1.4) implies that $H, \gamma, N$ and $\beta$ satisfy elliptic equation』 8

$$
\begin{aligned}
& \delta^{i k} \partial_{k} H_{i j}=-\frac{e^{2 \gamma}}{N}\left(2\left(e_{0} \phi\right)\left(\partial_{j} \phi\right)+\sum_{\mathbf{A}} F_{\mathbf{A}}^{2}\left(e_{0} u_{\mathbf{A}}\right)\left(\partial_{j} u_{\mathbf{A}}\right)\right) \\
& \Delta \gamma=-|\nabla \phi|^{2}-\frac{1}{2} \sum F_{\mathbf{A}}^{2}\left|\nabla u_{\mathbf{A}}\right|^{2}-\frac{e^{2 \gamma}}{N^{2}}\left(\left(e_{0} \phi\right)^{2}+\frac{1}{2} \sum_{\mathbf{A}} F_{\mathbf{A}}^{2}\left(e_{0} u_{\mathbf{A}}\right)^{2}\right)-\frac{1}{2} e^{-2 \gamma}|H|^{2}, \\
& \Delta N=N e^{-2 \gamma}|H|^{2}+\frac{e^{2 \gamma}}{N}\left(2\left(e_{0} \phi\right)^{2}+\sum_{\mathbf{A}} F_{\mathbf{A}}^{2}\left(e_{0} u_{\mathbf{A}}\right)^{2}\right) \\
& (L \beta)_{i j}=2 N e^{-2 \gamma} H_{i j}
\end{aligned}
$$

where $L$ is the conformal Killing operator given by

$$
(L \beta)_{i j}:=\delta_{j \ell} \partial_{i} \beta^{\ell}+\delta_{i \ell} \partial_{j} \beta^{\ell}-\delta_{i j} \partial_{k} \beta^{k} .
$$

3.2. Initial data. We recall in this section the notion of initial data that we introduce in [7. This applies for the system (1.4), and therefore in particular also for (1.3).

Definition 3.1 (Admissible initial data). For $-\frac{1}{2}<\delta<0, k \geq 3, R>0$ and $\mathcal{A}$ a finite set, an admissible initial data set with respect to the elliptic gauge for (1.4) consists of

(1) a conformally flat intrinsic metric $e^{2 \gamma} \delta_{i j}\left\lceil\Sigma_{0}\right.$ which admits a decomposition

$$
\gamma=-\gamma_{\text {asymp }} \chi(|x|) \log (|x|)+\widetilde{\gamma}
$$

where $\gamma_{\text {asymp }} \geq 0$ is a constant, $\chi(|x|)$ is a fixed smooth cutoff function with $\chi=0$ for $|x| \leq 1$ and $\chi=1$ for $|x| \geq 2$, and $\tilde{\gamma} \in H_{\delta}^{k+2}$;

(2) a second fundamental form $\left(H_{i j}\right) \uparrow_{\Sigma_{0}} \in H_{\delta+1}^{k+1}$ which is traceless;

(3) $\left(\frac{1}{N}\left(e_{0} \phi\right), \nabla \phi\right)\left\lceil\Sigma_{0} \in H^{k}\right.$, compactly supported in $B(0, R)$;

(4) $F_{\mathbf{A}}\left\lceil\Sigma_{0} \in H^{k}\right.$, compactly supported in $B(0, R)$ for every $\mathbf{A} \in \mathcal{A}$;

(5) $u_{\mathbf{A}}\left\lceil\Sigma_{0}\right.$ such that $\inf _{x \in \mathbb{R}^{2}} \mid \nabla u_{\mathbf{A}}\left\lceil\Sigma_{0} \mid(x)>C_{\text {eik }}^{-1}\right.$ for some $C_{\text {eik }}>0$ and $\left(\nabla u_{\mathbf{A}}\left\lceil_{\Sigma_{0}}-\overrightarrow{c_{\mathbf{A}}}\right) \in H_{\delta}^{k+1}\right.$, where $\overrightarrow{c_{\mathbf{A}}}$ is a constant vector field for every $\mathbf{A} \in \mathcal{A}$.

$\gamma$ and $H$ are required to satisfy the following constraint equations:

$$
\begin{aligned}
& \delta^{i k} \partial_{k} H_{i j}=-\frac{2 e^{2 \gamma}}{N}\left(e_{0} \phi\right) \partial_{j} \phi-\sum_{\mathbf{A}} e^{\gamma} F_{\mathbf{A}}^{2}\left|\nabla u_{\mathbf{A}}\right| \partial_{j} u_{\mathbf{A}}, \\
& \Delta \gamma+e^{-2 \gamma}\left(\frac{e^{4 \gamma}}{N^{2}}\left(e_{0} \phi\right)^{2}+\frac{1}{2}|H|^{2}\right)+|\nabla \phi|^{2}+\sum_{\mathbf{A}} F_{\mathbf{A}}^{2}\left|\nabla u_{\mathbf{A}}\right|^{2}=0 .
\end{aligned}
$$

As we show in [7] (following [6]), one can define a notion of "admissible free initial data", which consist of (rescaled versions of) data for the matter field, so that one can uniquely construct initial data set as in Definition 3.1 by solving the constraints. We recall this definition here:

Definition 3.2 (Admissible free initial data). Define $\dot{\phi}, \breve{F}_{\mathbf{A}}$ as follows:

$$
\dot{\phi}=\frac{e^{2 \gamma}}{N}\left(e_{0} \phi\right), \quad \breve{F}_{\mathbf{A}}=F_{\mathbf{A}} e^{\frac{\gamma}{2}},
$$

where $\gamma$ is as in (3.5).

For $-\frac{1}{2}<\delta<0, k \geq 3, R>0$ and $\mathcal{A}$ a finite set, an admissible free initial data set with respect to the elliptic gauge is given by the following:

(1) $(\dot{\phi}, \nabla \phi) \Gamma_{\Sigma_{0}} \in H^{k}$, compactly supported in $B(0, R)$;

(2) $\breve{F}_{\mathbf{A}}\left\lceil\Sigma_{0} \in H^{k}\right.$, compactly supported in $B(0, R)$ for every $\mathbf{A} \in \mathcal{A}$;

(3) $u_{\mathbf{A}}\left\lceil\Sigma_{0}\right.$ such that $\inf _{x \in \mathbb{R}^{2}} \mid \nabla u_{\mathbf{A}}\left\lceil\Sigma_{0} \mid(x)>C_{\text {eik }}^{-1}\right.$ for some $C_{\text {eik }}>0$ and $\left(\nabla u_{\mathbf{A}}\left\lceil\Sigma_{0}-\overrightarrow{c_{\mathbf{A}}}\right) \in H_{\delta}^{k+1}\right.$, where $\overrightarrow{c_{\mathbf{A}}}$ is a constant vector field for every $\mathbf{A} \in \mathcal{A}$.

Moreover, $\left.\left(\dot{\phi}, \nabla \phi, \breve{F}_{\mathbf{A}}, u_{\mathbf{A}}\right)\right|_{\Sigma_{0}}$ is required to satisfy

$$
\int_{\mathbb{R}^{2}}\left(-2 \dot{\phi} \partial_{j} \phi-\sum_{\mathbf{A}} \breve{F}_{\mathbf{A}}^{2}\left|\nabla u_{\mathbf{A}}\right| \partial_{j} u_{\mathbf{A}}\right) d x=0
$$

\footnotetext{
${ }^{8}$ This follows from the computations given in our companion paper [7, Appendix B].
} 
3.3. Local well-posedness. The following is our main result in [7] on the local well-posedness for (1.4) (and therefore also (1.3) ). While the theorem requires a smallness assumption (3.17), it is important to note that the smallness is not needed for the higher norms. This is important for the applications to Theorem 1.1 since the approximating solutions $g_{\lambda}$ that we construct necessarily oscillate with very high frequency 9

Theorem $3.3([7])$. Let $-\frac{1}{2}<\delta<0, k \geq 3, R>0$ and $\mathcal{A}$ be a finite set. Given a free initial data set as in Definition 3.2 such that

$$
\|\dot{\phi}\|_{L^{\infty}}+\|\nabla \phi\|_{L^{\infty}}+\max _{\mathbf{A}}\left\|\breve{F}_{\mathbf{A}}\right\|_{L^{\infty}} \leq \varepsilon
$$

and

$$
C_{e i k}:=\left(\min _{\mathbf{A}} \inf _{x \in \mathbb{R}^{2}}\left|\nabla u_{\mathbf{A}}\right|(x)\right)^{-1}+\max _{\mathbf{A}}\left\|\nabla u_{\mathbf{A}}-\overrightarrow{c_{\mathbf{A}}}\right\|_{H_{\delta}^{k+1}}<\infty,
$$

and

$$
C_{h i g h}:=\|\dot{\phi}\|_{H^{k}}+\|\nabla \phi\|_{H^{k}}+\left\|\breve{F}_{\mathbf{A}}\right\|_{H^{k}}<\infty .
$$

Then, for any $C_{\text {eik }}$ and $C_{\text {high }}$, there exists a constant $\varepsilon_{\text {low }}=\varepsilon_{\text {low }}\left(C_{\text {eik }}, k, \delta, R\right)>0$ independent of $C_{\text {high }}$ and a $T=T\left(C_{\text {high }}, C_{\text {eik }}, k, \delta, R\right)>0$ such that if $\varepsilon<\varepsilon_{\text {low }}$, there exists a unique solution to (1.4) in elliptic gauge on $[0, T] \times \mathbb{R}^{2}$. Moreover, the following holds for some constant $C_{h}=C_{h}\left(C_{\text {eik }}, C_{\text {high }}, k, \delta, R\right)>0$ :

- The following estimates hold for $\phi, F_{\mathbf{A}}$ and $u_{\mathbf{A}}$ for all $\mathbf{A} \in \mathcal{A}$ for $t \in[0, T]$ :

$$
\begin{array}{r}
\|\nabla \phi\|_{H^{k}}+\left\|\partial_{t} \phi\right\|_{H^{k}}+\left\|\partial_{t}^{2} \phi\right\|_{H^{k-1}} \leq C_{h}, \\
\max _{\mathbf{A}}\left(\left\|F_{\mathbf{A}}\right\|_{H^{k}}+\left\|\partial_{t} F_{\mathbf{A}}\right\|_{H^{k-1}}+\left\|\partial_{t}^{2} F_{\mathbf{A}}\right\|_{H^{k-2}}\right) \leq C_{h}, \\
\left(\min _{\mathbf{A}} \inf _{x \in \mathbb{R}^{2}}\left|\nabla u_{\mathbf{A}}\right|(x)\right)^{-1}+\max _{\mathbf{A}}\left(\left\|\nabla u_{\mathbf{A}}-\overrightarrow{c_{\mathbf{A}}}\right\|_{H_{\delta}^{k}}+\left\|e^{\gamma} N^{-1}\left(e_{0} u_{\mathbf{A}}\right)-\left|\overrightarrow{c_{\mathbf{A}}}\right|\right\|_{H_{\delta}^{k}}\right) \leq C_{h}, \\
\max _{\mathbf{A}}\left(\left\|\partial_{t} \nabla u_{\mathbf{A}}\right\|_{H_{\delta}^{k-1}}+\left\|\partial_{t}^{2} \nabla u_{\mathbf{A}}\right\|_{H_{\delta}^{k-2}}+\left\|\partial_{t}\left(\frac{e^{\gamma}}{N} e_{0} u_{\mathbf{A}}\right)\right\|_{H_{\delta}^{k-1}}+\left\|\partial_{t}^{2}\left(\frac{e^{\gamma}}{N} e_{0} u_{\mathbf{A}}\right)\right\|_{H_{\delta}^{k-2}}\right) \leq C_{h} .
\end{array}
$$

- The metric components $\gamma$ and $N$ can be decomposed as

$$
\gamma=\gamma_{\text {asymp }} \chi(|x|) \log (|x|)+\widetilde{\gamma}, \quad N=1+N_{\text {asymp }}(t) \chi(|x|) \log (|x|)+\tilde{N}
$$

with $\gamma_{\text {asymp }} \leq 0$ a constant, $N_{\text {asymp }}(t) \geq 0$ a function of $t$ alone and $\chi(|x|)$ is a fixed smooth, non-negative cutoff function supported in $\{|x| \geq 1\}$ which is identically 1 for $|x| \geq 2$.

- $\gamma, N$ and $\beta$ obey the following estimates for $t \in[0, T]$ :

$$
\begin{aligned}
\left|\gamma_{\text {asymp }}\right|+\|\widetilde{\gamma}\|_{H_{\delta}^{k+2}}+\left\|\partial_{t} \widetilde{\gamma}\right\|_{H_{\delta}^{k+1}}+\left\|\partial_{t}^{2} \widetilde{\gamma}\right\|_{H_{\delta}^{k}} \leq C_{h}, \\
\left|N_{\text {asymp }}\right|+\left|\partial_{t} N_{\text {asymp }}\right|+\left|\partial_{t}^{2} N_{\text {asymp }}\right| \leq C_{h}, \\
\|\widetilde{N}\|_{H_{\delta}^{k+2}}+\left\|\partial_{t} \widetilde{N}\right\|_{H_{\delta}^{k+1}}+\left\|\partial_{t}^{2} \widetilde{N}\right\|_{H_{\delta}^{k}} \leq C_{h}, \\
\|\beta\|_{H_{\delta}^{k+2}}+\left\|\partial_{t} \beta\right\|_{H_{\delta}^{k+1}}+\left\|\partial_{t}^{2} \beta\right\|_{H_{\delta}^{k}} \leq C_{h} .
\end{aligned}
$$

- The support of $\phi$ and $F_{\mathbf{A}}$ satisfie 10

$$
\operatorname{supp}\left(\phi, F_{\mathbf{A}}\right) \subset J^{+}\left(\Sigma_{0} \cap B(0, R)\right) .
$$

Remark 3.4. From now on, let us fix a cutoff function $\chi$ with the properties as in the statement of Theorem 3.3 .

Finally, as discussed in [7, when specialized in the case that the data are genuinely small - in the sense that even the high norms are small - the time of existence can be taken to be $T=1$ :

Corollary 3.5 ([7]). Suppose the assumptions of Theorem 3.3 hold and let $\left\{c_{\mathbf{A}}\right\}_{\mathbf{A} \in \mathcal{A}}$ be a collection of constant vector fields on the plane. There exists $\varepsilon_{\text {small }}=\varepsilon_{\text {small }}\left(\delta, k, R, c_{\mathbf{A}}\right)$ such that if $C_{\text {high }}$ and $\varepsilon$ in Theorem 3.3 both satisfy

$$
C_{\text {high }}, \varepsilon \leq \varepsilon_{\text {small }}
$$

\footnotetext{
${ }^{9}$ We note that in the statement of Theorem 3.3 in [7, different components of the metric are in spaces of different weight. While that is useful for the proof of Theorem 3.3 it is irrelevant for this paper. We simply take the worst weight in the norms in 7] so that the decay part of the metric components $\gamma, N$ and $\beta$ are all in $H_{\delta}^{k+2}$.

${ }^{10}$ Here, $J^{+}$denotes the causal future.
} 
and moreover

$$
\sum_{\mathbf{A}}\left\|\nabla u_{\mathbf{A}}-\overrightarrow{c_{\mathbf{A}}}\right\|_{H_{\delta}^{k+1}} \leq \varepsilon_{\text {small }}
$$

then the unique solution exists in $[0,1] \times \mathbb{R}^{2}$. Moreover, there exists $C_{0}=C_{0}\left(\delta, k, R, c_{\mathbf{A}}\right)$ such that all the estimates in Theorem 3.3 hold with $C_{h}$ replaced by $C_{0} \varepsilon$.

\section{MAin RESUlT}

\subsection{Second version of the main theorem.}

Definition 4.1. Given a solution to (1.4) on $I \times \mathbb{R}^{2}$ for $I \subset \mathbb{R}$ (which is as regular as that in Theorem 3.3), we say that the set of eikonal functions $\left\{u_{\mathbf{A}}\right\}_{\mathbf{A} \in \mathcal{A}}$ is angularly separated if there exists $\eta^{\prime} \in(0,1)$ such that

$$
\frac{\delta^{i j}\left(\partial_{i} u_{\mathbf{A}_{1}}\right)\left(\partial_{j} u_{\mathbf{A}_{2}}\right)}{\left|\nabla u_{\mathbf{A}_{1}}\right|\left|\nabla u_{\mathbf{A}_{2}}\right|}(t, x)<1-\eta^{\prime}, \quad \forall(t, x) \in I \times \mathbb{R}^{2}, \quad \forall \mathbf{A}_{1} \neq \mathbf{A}_{2} .
$$

Theorem 4.2 (Main theorem). Le $1110,-\frac{1}{2}<\delta<0, R>0$ and $\mathcal{A}$ be a finite set. Given an admissible free initial data set $\left(\dot{\phi}, \nabla \phi, \breve{F}_{\mathbf{A}}, u_{\mathbf{A}}\right)\left\lceil\Sigma_{0}\right.$ such that

$$
\mid \nabla u_{\mathbf{A}}\left\lceil\Sigma_{0} \mid>\frac{1}{2}\right.
$$

- $u_{\mathbf{A}} \Gamma_{\Sigma_{0}}$ is angularly separated 12 ;

- (Smallness condition)

$$
\left\|\nabla u_{\mathbf{A}}-\overrightarrow{c_{\mathbf{A}}}\right\|_{H_{\delta}^{11}}+\|\dot{\phi}\|_{H^{10}}+\|\nabla \phi\|_{H^{10}}+\left\|\breve{F}_{\mathbf{A}}\right\|_{H^{10}} \leq \varepsilon
$$

- (Genericity condition on initial data) there exists a point $p \in \mathbb{R}^{2}$ such that

$$
\left(\begin{array}{c}
\left(\partial_{1} \phi_{0}\right)\left\lceil\Sigma_{0}\right. \\
\left(\partial_{1} \dot{\phi}_{0}\right)\left\lceil\Sigma_{0}\right.
\end{array}\right)(p) \text { and }\left(\begin{array}{c}
\left(\partial_{2} \phi_{0}\right)\left\lceil\Sigma_{0}\right. \\
\left(\partial_{2} \dot{\phi}_{0}\right)\left\lceil\Sigma_{0}\right.
\end{array}\right)(p) \text { are linearly independent. }
$$

Then, there exists $\varepsilon_{0}>0$ such that if $\varepsilon<\varepsilon_{0}$, a unique solution $\left(g_{0}, \phi_{0},\left(F_{0}\right)_{\mathbf{A}},\left(u_{0}\right)_{\mathbf{A}}\right)$ to (1.4) arising from the given admissible free initial data set exists on a time interval $[0,1]$, and there exists a one-parameter family of solutions $\left(g_{\lambda}, \phi_{\lambda}\right)$ to (1.3) for $\lambda \in\left(0, \lambda_{0}\right)$ (for some $\lambda_{0} \in \mathbb{R}$ sufficiently small), which are all defined on the time interval $[0,1]$, such that

$$
\left(g_{\lambda}, \phi_{\lambda}\right) \rightarrow\left(g_{0}, \phi_{0}\right) \text { uniformly on compact sets }
$$

and

$$
\left(\partial g_{\lambda}, \partial \phi_{\lambda}\right) \rightarrow\left(\partial g_{0}, \partial \phi_{0}\right) \text { weakly in } L^{2}
$$

with $\partial g_{\lambda}, \partial \phi_{\lambda} \in L_{l o c}^{\infty}$ uniformly.

Remark 4.3. As we will see (for instance by considering (4.24) and the estimates for $\mathfrak{g}$ ), $\partial g_{\lambda}$ converges in a stronger sense compared to $\partial \phi_{\lambda}$. In particular, $\partial g_{\lambda} \rightarrow \partial g_{0}$ uniformly (in $L^{\infty}$ ) on compact sets (of $\left.[0,1] \times \mathbb{R}^{2}\right)$.

Clearly the existence and uniqueness of $\left(g_{0}, \phi_{0},\left(F_{0}\right)_{\mathbf{A}},\left(u_{0}\right)_{\mathbf{A}}\right)$ - which from now on will be called the background solution - follows from Corollary 3.5. We therefore have to prove the existence of solutions $\left(g_{\lambda}, \phi_{\lambda}\right)$ to (1.3) and their convergence, for some appropriately chosen initial data (cf. Lemma 4.9). Since $F_{\mathbf{A}} \equiv 0$ for the solutions to (1.3), we will from now on denote $F_{\mathbf{A}}=\left(F_{0}\right)_{\mathbf{A}}$ and $u_{\mathbf{A}}=\left(u_{0}\right)_{\mathbf{A}}$ to lighten the notations.

\footnotetext{
${ }^{11}$ Note that while the constants $k, \delta$ and $R$ do not appear explicitly in the statement of the theorem, they are needed in the definition of admissible free initial data set.

${ }^{12}$ Strictly speaking, angular separation was defined for spacetimes on $I \times \mathbb{R}^{2}$, where $I$ in an interval, but it is easy to extend its definition to include the case $I=\{0\}$.
} 
4.2. Angularly separated, spatially adapted and null adapted eikonal functions. In Theorem 4.2 . the eikonal functions $\left\{u_{\mathbf{A}}\right\}_{\mathbf{A} \in \mathcal{A}}$ are required to be initially angularly separated with $\left|\nabla u_{\mathbf{A}}\right| \Sigma_{0} \mid>\frac{1}{2}$. It is easy to see that by choosing $\varepsilon_{0}$ in Theorem 4.2 sufficiently small, $\left\{u_{\mathbf{A}}\right\}_{\mathbf{A} \in \mathcal{A}}$ is in fact angularly separated in $[0,1] \times \mathbb{R}^{2}$ with the lower bound $\left|\nabla u_{\mathbf{A}}\right|>\frac{1}{4}$ (cf. Lemma 4.4). However, in order to carry out the proof of Theorem 4.2 (and in particular to construct the parametrix), it will be convenient to modify the eikonal functions such that they satisfy the additional properties of being spatially adapted and null adapted (see Definitions 4.6 and 4.7 below). That this can always be achieved is proven in Lemma 4.8.

We begin our discussion with the following lemma, which states that our assumptions on $\left\{u_{\mathbf{A}}\right\}_{\mathbf{A} \in \mathcal{A}}$ in Theorem 4.2 together with the smallness assumption, give us good control of $\left\{u_{\mathbf{A}}\right\}_{\mathbf{A} \in \mathcal{A}}$ in the spacetime.

Lemma 4.4. Under the assumptions of Theorem 4.2. if $\varepsilon_{0}$ is sufficiently small, the eikonal functions $\left\{u_{\mathbf{A}}\right\}_{\mathbf{A} \in \mathcal{A}}$ are angularly separated ${ }^{13}$ in $[0,1] \times \mathbb{R}^{2}$ and satisfy $\left|\nabla u_{\mathbf{A}}\right|>\frac{1}{4}$.

Proof. This can be easily justified using the estimates in Corollary 3.5.

We now discuss the modifications to the eikonal functions. To begin, we make the easy observation that the following symmetry of the system (1.4) allows us to modify the eikonal functions:

Lemma 4.5. Suppose $\left(g, \phi, F_{\mathbf{A}}, u_{\mathbf{A}}\right)$ is a solution to (1.4) on $I \times \mathbb{R}^{2}$ for $I \subset \mathbb{R}$ in the sense of Theorem 3.3. For any sets of positive constants $\left\{c_{\mathbf{A}}\right\}_{\mathbf{A} \in \mathcal{A}} \in \mathbb{R}_{>0}^{|\mathcal{A}|}$, if we define

$$
F_{\mathbf{A}}^{\prime}=c_{\mathbf{A}}^{-1} F_{\mathbf{A}}, \quad u_{\mathbf{A}}^{\prime}=c_{\mathbf{A}} u_{\mathbf{A}},
$$

then $\left(g, \phi, F_{\mathbf{A}}^{\prime}, u_{\mathbf{A}}^{\prime}\right)$ is also a solution to (1.4).

We now define the notions of spatially adapted and null adapted eikonal functions:

Definition 4.6. Given a solution to (1.4) on $I \times \mathbb{R}^{2}$ for $I \subset \mathbb{R}$ (which is as regular as that in Theorem 3.3), we say that the set of eikonal functions $\left\{u_{\mathbf{A}}\right\}_{\mathbf{A} \in \mathcal{A}^{\prime}}$ is spatially adapted for $\mathcal{A}^{\prime} \subset \mathcal{A}$ if

(1) $\left|\nabla u_{\mathbf{A}}\right|>\frac{1}{4}, \quad \forall \mathbf{A} \in \mathcal{A}$,

(2) $\left|\nabla\left(u_{\mathbf{A}_{1}} \pm u_{\mathbf{A}_{2}}\right)\right|>\frac{1}{4}, \quad \forall \mathbf{A}_{1}, \mathbf{A}_{2} \in \mathcal{A}^{\prime}, \mathbf{A}_{1} \neq \mathbf{A}_{2}$,

(3) $\left|\nabla\left(u_{\mathbf{A}_{1}} \pm 2 u_{\mathbf{A}_{2}}\right)\right|>\frac{1}{4}, \quad \forall \mathbf{A}_{1}, \mathbf{A}_{2} \in \mathcal{A}^{\prime}, \mathbf{A}_{1} \neq \mathbf{A}_{2}$,

(4) $\left|\nabla\left(u_{\mathbf{A}_{1}} \pm 3 u_{\mathbf{A}_{2}}\right)\right|>\frac{1}{4}, \quad \forall \mathbf{A}_{1}, \mathbf{A}_{2} \in \mathcal{A}^{\prime}, \mathbf{A}_{1} \neq \mathbf{A}_{2}$.

Definition 4.7. Given a solution to (1.4) on $I \times \mathbb{R}^{2}$ for $I \subset \mathbb{R}$ (which is as regular as that in Theorem 3.3), we say that the set of eikonal functions $\left\{u_{\mathbf{A}}\right\}_{\mathbf{A} \in \mathcal{A}^{\prime}}$ is null adapted for $\mathcal{A}^{\prime} \subset \mathcal{A}$ if there exists a constant $\eta>0$ such that for any choice of the signs $\pm, \pm_{1}, \pm_{2}$, it holds tha 15

(1) $\left.\mid\left(g^{-1}\right)^{\alpha \beta} \partial_{\alpha}\left(u_{\mathbf{A}_{1}} \pm u_{\mathbf{A}_{2}}\right) \partial_{\beta}\left(u_{\mathbf{A}_{1}} \pm u_{\mathbf{A}_{2}}\right)\right) \mid>\eta e^{-2 \gamma}, \quad \forall \mathbf{A}_{1}, \mathbf{A}_{2} \in \mathcal{A}^{\prime}, \mathbf{A}_{1} \neq \mathbf{A}_{2}$,

(2) $\left.\mid\left(g^{-1}\right)^{\alpha \beta} \partial_{\alpha}\left(u_{\mathbf{A}_{1}} \pm 2 u_{\mathbf{A}_{2}}\right) \partial_{\beta}\left(u_{\mathbf{A}_{1}} \pm 2 u_{\mathbf{A}_{2}}\right)\right) \mid>\eta e^{-2 \gamma}, \quad \forall \mathbf{A}_{1}, \mathbf{A}_{2} \in \mathcal{A}^{\prime}, \mathbf{A}_{1} \neq \mathbf{A}_{2}$,

(3) $\left.\mid\left(g^{-1}\right)^{\alpha \beta} \partial_{\alpha}\left(u_{\mathbf{A}_{1}} \pm_{1} u_{\mathbf{A}_{2}} \pm_{2} u_{\mathbf{A}_{3}}\right) \partial_{\beta}\left(u_{\mathbf{A}_{1}} \pm_{1} u_{\mathbf{A}_{2}} \pm_{2} u_{\mathbf{A}_{3}}\right)\right) \mid>\eta e^{-2 \gamma}, \quad \forall \mathbf{A}_{1}, \mathbf{A}_{2}, \mathbf{A}_{3} \in \mathcal{A}^{\prime}$ all different.

Lemma 4.8. Given a solution to (1.4) on $I \times \mathbb{R}^{2}$ for $I \subset \mathbb{R}$ (which is as regular as that in Theorem 3.3) such that the eikonal functions $\left\{u_{\mathbf{A}}\right\}_{\mathbf{A} \in \mathcal{A}}$ are angularly separated with $\left|\nabla u_{\mathbf{A}}\right|>\frac{1}{4}, \forall \mathbf{A} \in \mathcal{A}$, there exists a collection of positive constants $\left\{c_{\mathbf{A}}\right\}_{\mathbf{A} \in \mathcal{A}}$ such that for $u_{\mathbf{A}}^{\prime}=c_{\mathbf{A}} u_{\mathbf{A}},\left\{u_{\mathbf{A}}^{\prime}\right\}_{\mathbf{A} \in \mathcal{A}}$ is spatially adapted and null adapted.

Proof. We order the eikonal functions as $u_{\mathbf{A}_{1}}, u_{\mathbf{A}_{2}}, \ldots, u_{\mathbf{A}_{N}}$, where $N=|\mathcal{A}|$. We then proceed inductively on $m$ to show that

(1) The set $\cup_{i=1}^{m}\left\{u_{\mathbf{A}_{i}}^{\prime}\right\}$ (where $u_{\mathbf{A}_{i}}^{\prime}=c_{\mathbf{A}_{i}} u_{\mathbf{A}_{i}}$ ) is both spatially adapted and null adapted.

(2) For every $i_{1}<i_{2} \leq m$ and $j>m$, the following inequality holds (notice that the following expression involves the primed $u^{\prime}$ for $i_{1}, i_{2}$ and the unprimed $u$ for $j$ ):

$$
\left|\nabla u_{\mathbf{A}_{i_{2}}}^{\prime}\right|\left|\nabla u_{\mathbf{A}_{j}}\right|-\nabla u_{\mathbf{A}_{i_{2}}}^{\prime} \cdot \nabla u_{\mathbf{A}_{j}} \geq 2\left(\left|\nabla u_{\mathbf{A}_{i_{1}}}^{\prime}\right| \nabla u_{\mathbf{A}_{j}} \mid-\nabla u_{\mathbf{A}_{i_{1}}}^{\prime} \cdot \nabla u_{\mathbf{A}_{j}}\right) .
$$

Once we have completed this induction, the lemma follows from the case $m=N$.

Notice that the base case where $m=1$ is trivial. In the following, we will carry out the inductive step, assuming that $c_{\mathbf{A}_{i}}, \ldots, c_{\mathbf{A}_{m-1}}$ have been chosen such that (1) and (2) above hold with $m$ replaced by $m-1$. We will then choose $c_{\mathbf{A}_{m}}>0$ such that (1) and (2) hold.

\footnotetext{
${ }^{13}$ Notice that in $[0,1] \times \mathbb{R}^{2}$ the estimate in Definition 4.1 may hold with a different constant compared to the initial data, but we will call both of these constants $\eta^{\prime}$

${ }^{14}$ We choose $c_{\mathbf{A}}$ to be positive so that we also preserve the property that $\left(e_{0} u_{\mathbf{A}}\right)>0$.

${ }^{15}$ Here, $\gamma$ is as in 3.5.
} 
Proof of (4.2). By the induction hypothesis, it suffices to prove (4.2) with $i_{2}=m$ and with any $i_{1}=i<m, j>m$. By the assumption on angular separation together with the lower bounds on $\left|\nabla u_{\mathbf{A}}\right|$, we have

$$
\left|\nabla u_{\mathbf{A}_{m}}^{\prime}\right|\left|\nabla u_{\mathbf{A}_{j}}\right|-\nabla u_{\mathbf{A}_{m}}^{\prime} \cdot \nabla u_{\mathbf{A}_{j}}=c_{\mathbf{A}_{m}}\left(\left|\nabla u_{\mathbf{A}_{m}}\right|\left|\nabla u_{\mathbf{A}_{j}}\right|-\nabla u_{\mathbf{A}_{m}} \cdot \nabla u_{\mathbf{A}_{j}}\right)>\frac{c_{\mathbf{A}_{m}} \eta^{\prime}}{16} .
$$

On the other hand, since $c_{\mathbf{A}_{i}}$ has been fixed, $\left|\nabla u_{\mathbf{A}_{i}}^{\prime}\right|\left|\nabla u_{\mathbf{A}_{j}}\right|-\nabla u_{\mathbf{A}_{i}}^{\prime} \cdot \nabla u_{\mathbf{A}_{j}}$ is bounded above. We can therefore choose

$$
c_{\mathbf{A}_{m}} \geq \frac{2 \max _{i<m<j} \sup _{\mathbb{R}^{2}}\left|\nabla u_{\mathbf{A}_{i}}^{\prime}\right|\left|\nabla u_{\mathbf{A}_{j}}\right|}{\min _{j>m} \inf _{\mathbb{R}^{2}}\left(\left|\nabla u_{\mathbf{A}_{m}}^{\prime}\right|\left|\nabla u_{\mathbf{A}_{j}}\right|-\nabla u_{\mathbf{A}_{m}}^{\prime} \cdot \nabla u_{\mathbf{A}_{j}}\right)}
$$

so that

$$
\begin{aligned}
\left|\nabla u_{\mathbf{A}_{m}}^{\prime}\right|\left|\nabla u_{\mathbf{A}_{j}}\right|-\nabla u_{\mathbf{A}_{m}}^{\prime} \cdot \nabla u_{\mathbf{A}_{j}} & =c_{\mathbf{A}_{m}}\left(\left|\nabla u_{\mathbf{A}_{m}}\right|\left|\nabla u_{\mathbf{A}_{j}}\right|-\nabla u_{\mathbf{A}_{m}} \cdot \nabla u_{\mathbf{A}_{j}}\right) \\
& \geq 2\left(\left|\nabla u_{\mathbf{A}_{i}}^{\prime}\right|\left|\nabla u_{\mathbf{A}_{j}}\right|-\nabla u_{\mathbf{A}_{i}}^{\prime} \cdot \nabla u_{\mathbf{A}_{j}}\right),
\end{aligned}
$$

i.e., (4.2) holds for $i_{2}=m, i_{1}-i<m$ and $j>m$.

Spatially adapted. By the induction hypothesis, we only need to verify the conditions in Definition [4.6 when one of the eikonal functions involved is $u_{\mathbf{A}_{m}}$.

First, it is easy to see that if

$$
c_{\mathbf{A}_{m}}>1,
$$

Then $\left|\nabla u_{\mathbf{A}_{m}}^{\prime}\right|>\frac{1}{4}$.

Next, we compute that for $i<m$

$$
\begin{aligned}
& \left|\nabla\left(u_{\mathbf{A}_{i}}^{\prime} \pm u_{\mathbf{A}_{m}}^{\prime}\right)\right|^{2} \geq c_{\mathbf{A}_{m}}^{2}\left|\nabla u_{\mathbf{A}_{m}}\right|^{2}-2 c_{\mathbf{A}_{m}}\left|\nabla u_{\mathbf{A}_{m}}\right|\left|\nabla u_{\mathbf{A}_{i}}^{\prime}\right|, \\
& \left|\nabla\left(u_{\mathbf{A}_{i}}^{\prime} \pm 2 u_{\mathbf{A}_{m}}^{\prime}\right)\right|^{2} \geq 4 c_{\mathbf{A}_{m}}^{2}\left|\nabla u_{\mathbf{A}_{m}}\right|^{2}-4 c_{\mathbf{A}_{m}}\left|\nabla u_{\mathbf{A}_{m}}\right|\left|\nabla u_{\mathbf{A}_{i}}^{\prime}\right|, \\
& \left|\nabla\left(2 u_{\mathbf{A}_{i}}^{\prime} \pm u_{\mathbf{A}_{m}}^{\prime}\right)\right|^{2} \geq c_{\mathbf{A}_{m}}^{2}\left|\nabla u_{\mathbf{A}_{m}}\right|^{2}-4 c_{\mathbf{A}_{m}}\left|\nabla u_{\mathbf{A}_{m}}\right|\left|\nabla u_{\mathbf{A}_{i}}^{\prime}\right|, \\
& \left|\nabla\left(u_{\mathbf{A}_{i}}^{\prime} \pm 3 u_{\mathbf{A}_{m}}^{\prime}\right)\right|^{2} \geq 9 c_{\mathbf{A}_{m}}^{2}\left|\nabla u_{\mathbf{A}_{m}}\right|^{2}-6 c_{\mathbf{A}_{m}}\left|\nabla u_{\mathbf{A}_{m}}\right|\left|\nabla u_{\mathbf{A}_{i}}^{\prime}\right|, \\
& \left|\nabla\left(3 u_{\mathbf{A}_{i}}^{\prime} \pm u_{\mathbf{A}_{m}}^{\prime}\right)\right|^{2} \geq c_{\mathbf{A}_{m}}^{2}\left|\nabla u_{\mathbf{A}_{m}}\right|^{2}-6 c_{\mathbf{A}_{m}}\left|\nabla u_{\mathbf{A}_{m}}\right|\left|\nabla u_{\mathbf{A}_{i}}^{\prime}\right| .
\end{aligned}
$$

Each of these is required to be $>\frac{1}{16}$. Using the fact that $\inf _{\mathbb{R}^{2}}\left|\nabla u_{\mathbf{A}_{m}}\right| \geq \frac{1}{4}$, we can therefore choose

$$
c_{\mathbf{A}_{m}} \geq \frac{12 \max _{i<m} \sup _{\mathbb{R}^{2}}\left|\nabla u_{\mathbf{A}_{i}}^{\prime}\right|}{\inf _{\mathbb{R}^{2}}\left|\nabla u_{\mathbf{A}_{m}}\right|}+2
$$

such that for the RHS of each line in (4.5)

$$
\begin{aligned}
& R H S \\
\geq & c_{\mathbf{A}_{m}}^{2}\left|\nabla u_{\mathbf{A}_{m}}\right|^{2}-6 c_{\mathbf{A}_{m}}\left|\nabla u_{\mathbf{A}_{m}}\right|\left|\nabla u_{\mathbf{A}_{i}}\right| \\
> & c_{\mathbf{A}_{m}}^{2}\left|\nabla u_{\mathbf{A}_{m}}\right|^{2}-\frac{c_{\mathbf{A}_{m}}^{2}}{2}\left|\nabla u_{\mathbf{A} m}\right| \inf _{\mathbb{R}^{2}}\left|\nabla u_{\mathbf{A}_{m}}\right| \\
\geq & \frac{1}{2} c_{\mathbf{A}_{m}}^{2}\left|\nabla u_{\mathbf{A}_{m}}\right|^{2} \geq \frac{1}{16} .
\end{aligned}
$$

Null adapted: the easy cases. By the induction hypothesis, in order to verify that $\cup_{i=1}^{m}\left\{u_{\mathbf{A}_{i}}^{\prime}\right\}$ is null adapted, we only need to check the conditions in Definition 4.7 where one of the eikonal functions is $u_{\mathbf{A}_{m}}$. We first check the first two conditions in Definition 4.7 which are easier and they hold for any choice of $c_{\mathbf{A}_{m}}>0$.

As a preliminary step, note that for any $\mathbf{A}$, since $u_{\mathbf{A}}$ is an eikonal function, by (3.5), we have $e_{0} u_{\mathbf{A}}=$ $N e^{-\gamma}\left|\nabla u_{\mathbf{A}}\right|$. Therefore, we compute for the sum (or difference) of two eikonal functions with $i<m$

$$
\begin{aligned}
& \left(g^{-1}\right)^{\alpha \beta} \partial_{\alpha}\left(u_{\mathbf{A}_{i}}^{\prime} \pm u_{\mathbf{A}_{m}}^{\prime}\right) \partial_{\beta}\left(u_{\mathbf{A}_{i}}^{\prime} \pm u_{\mathbf{A}_{m}}^{\prime}\right) \\
= & -\frac{1}{N^{2}}\left(e_{0}\left(u_{\mathbf{A}_{i}}^{\prime} \pm u_{\mathbf{A}_{m}}^{\prime}\right)\right)\left(e_{0}\left(u_{\mathbf{A}_{i}}^{\prime} \pm u_{\mathbf{A}_{m}}^{\prime}\right)\right)+e^{-2 \gamma} \nabla\left(u_{\mathbf{A}_{i}}^{\prime} \pm u_{\mathbf{A}_{m}}^{\prime}\right) \cdot \nabla\left(u_{\mathbf{A}_{i}}^{\prime} \pm u_{\mathbf{A}_{m}}^{\prime}\right) \\
= & 2 e^{-2 \gamma}\left(\mp\left|\nabla u_{\mathbf{A}_{i}}^{\prime}\right|\left|\nabla u_{\mathbf{A}_{m}}^{\prime}\right| \pm \nabla u_{\mathbf{A}_{i}}^{\prime} \cdot \nabla u_{\mathbf{A}_{m}}^{\prime}\right) .
\end{aligned}
$$


By Cauchy-Schwarz, Definition 4.1 and the assumption $\left|\nabla u_{\mathbf{A}_{i}}^{\prime}\right| \geq \frac{1}{4},\left|\nabla u_{\mathbf{A}_{m}}\right| \geq \frac{1}{4}$,

$$
\begin{aligned}
& |\mp| \nabla u_{\mathbf{A}_{i}}^{\prime}|| \nabla u_{\mathbf{A}_{m}}^{\prime}\left| \pm \nabla u_{\mathbf{A}_{i}}^{\prime} \cdot \nabla u_{\mathbf{A}_{m}}^{\prime}\right| \\
\geq & \left|\nabla u_{\mathbf{A}_{i}}^{\prime}\right|\left|\nabla u_{\mathbf{A}_{m}}^{\prime}\right|-\nabla u_{\mathbf{A}_{i}}^{\prime} \cdot \nabla u_{\mathbf{A}_{m}}^{\prime} \\
\geq & c_{\mathbf{A}_{m}} \eta^{\prime}\left|\nabla u_{\mathbf{A}_{i}}^{\prime}\right|\left|\nabla u_{\mathbf{A}_{m}}\right| \geq \frac{c_{\mathbf{A}_{m}} \eta^{\prime}}{16} .
\end{aligned}
$$

This verifies the first condition in Definition 4.7 for any $c_{\mathbf{A}_{m}} \geq 1$. The second condition in Definition 4.7 can be checked in an identical manner.

Null adapted: the hard case. It remains to check the condition in Definition 4.7 involving three distinct eikonal function. By the induction hypothesis, it suffices to consider $i<k<m$, in which case we compute

$$
\begin{aligned}
& \left.\left(g^{-1}\right)^{\alpha \beta} \partial_{\alpha}\left(u_{\mathbf{A}_{i}}^{\prime} \pm_{1} u_{\mathbf{A}_{k}}^{\prime} \pm_{2} u_{\mathbf{A}_{m}}^{\prime}\right) \partial_{\beta}\left(u_{\mathbf{A}_{i}}^{\prime} \pm_{1} u_{\mathbf{A}_{k}}^{\prime} \pm_{2} u_{\mathbf{A}_{m}}^{\prime}\right)\right) \\
= & -\frac{1}{N^{2}}\left(e_{0}\left(u_{\mathbf{A}_{i}}^{\prime} \pm_{1} u_{\mathbf{A}_{k}}^{\prime} \pm_{2} u_{\mathbf{A}_{m}}^{\prime}\right)\right)\left(e_{0}\left(u_{\mathbf{A}_{i}}^{\prime} \pm_{1} u_{\mathbf{A}_{k}}^{\prime} \pm_{2} u_{\mathbf{A}_{m}}^{\prime}\right)\right) \\
& +e^{-2 \gamma} \nabla\left(u_{\mathbf{A}_{i}}^{\prime} \pm_{1} u_{\mathbf{A}_{k}}^{\prime} \pm_{2} c_{\mathbf{A}_{m}} u_{\mathbf{A}_{m}}\right) \cdot \nabla\left(u_{\mathbf{A}_{i}}^{\prime} \pm_{1} u_{\mathbf{A}_{k}}^{\prime} \pm_{2} c_{\mathbf{A}_{m}} u_{\mathbf{A}_{m}}\right) \\
= & 2 e^{-2 \gamma} \underbrace{\left(\mp 1\left|\nabla u_{\mathbf{A}_{i}}^{\prime}\right|\left|\nabla u_{\mathbf{A}_{k}^{\prime}}\right| \pm_{1} \nabla u_{\mathbf{A}_{i}}^{\prime} \cdot \nabla u_{\mathbf{A}_{k}}^{\prime}\right)}_{=: I} \\
& +2 c_{\mathbf{A}_{m}} e^{-2 \gamma} \underbrace{\left(\mp_{2}\left|\nabla u_{\mathbf{A}_{i}}^{\prime}\right|\left|\nabla u_{\mathbf{A}_{m}}\right| \pm_{2} \nabla u_{\mathbf{A}_{i}}^{\prime} \cdot \nabla u_{\mathbf{A}_{m}}\right)}_{=: I I} \\
& +2 c_{\mathbf{A}_{m}} e^{-2 \gamma} \underbrace{\left(\mp_{1} \mp_{2}\left|\nabla u_{\mathbf{A}_{k}}^{\prime}\right|\left|\nabla u_{\mathbf{A}_{m}}\right| \mp_{1} \pm_{2} \nabla u_{\mathbf{A}_{k}}^{\prime} \cdot \nabla u_{\mathbf{A}_{m}}\right)}_{=: I I} .
\end{aligned}
$$

Let us note that by the angular separation assumption, each of $I, I I$ and $I I I$ are bounded below. It remains to check that not too much cancellations can occur. The key is to note that by the induction hypothesis (more precisely, by (4.2) with $m$ replaced by $m-1$ ), we have

$$
|I I+I I I| \geq\left|\nabla u_{\mathbf{A}_{i}}^{\prime}\right|\left|\nabla u_{\mathbf{A}_{m}}\right|-\nabla u_{\mathbf{A}_{i}}^{\prime} \cdot \nabla u_{\mathbf{A}_{m}},
$$

which, by the angular separation assumption and the lower bound on $\left|\nabla u_{\mathbf{A}}\right|$, is uniformly bounded below for any $i<m$. Therefore, by choosing

$$
c_{\mathbf{A}_{m}} \geq \frac{2 \max _{i<k<m} \sup _{\mathbb{R}^{2}}\left|\nabla u_{\mathbf{A}_{i}}^{\prime}\right|\left|\nabla u_{\mathbf{A}_{k}}^{\prime}\right|}{\min _{i<m} \inf _{\mathbb{R}^{2}}\left|\nabla u_{\mathbf{A}_{i}}^{\prime}\right|\left|\nabla u_{\mathbf{A}_{m}}\right|-\nabla u_{\mathbf{A}_{i}}^{\prime} \cdot \nabla u_{\mathbf{A}_{m}}},
$$

(4.8) is bounded below as follows

$$
\text { (4.8) } \geq 2 e^{-2 \gamma} \max _{i<k<m} \sup _{\mathbb{R}^{2}}\left|\nabla u_{\mathbf{A}_{i}}^{\prime}\right|\left|\nabla u_{\mathbf{A}_{k}}^{\prime}\right| \geq \frac{1}{8} e^{-2 \gamma},
$$

and therefore satisfies the required condition.

End of proof. To conclude the induction step, it is easy to see that $c_{\mathbf{A}_{m}}$ can be chosen such that (4.3), (4.4), (4.6) and (4.9) are simultaneously verified. This then gives the desired result.

From now on, we assume that the eikonal functions $u_{\mathrm{A}}$ with respect to the background solution are both spatially adapted and null adapted.

4.3. Construction of the free initial data for $\left(g_{\lambda}, \phi_{\lambda}\right)$. We construct the one parameter family $\left(g_{\lambda}, \phi_{\lambda}\right)$ by specifying appropriate initial data to (1.3). In the remainder of the paper, we will prove that the solutions, guaranteed to exist locally by Theorem 3.3 . in fact exist throughout the time interval $[0,1]$ and have the desired convergence properties as $\lambda \rightarrow 0$. The following lemma constructs the initial data:

Lemma 4.9. Under the assumptions of Theorem 4.2. there exists $\lambda_{0}>0$ sufficiently small such that for every $\lambda \in\left(0, \lambda_{0}\right]$, there exist initial functions $\phi_{\lambda}\left\lceil\Sigma_{0}\right.$ and $\dot{\phi}_{\lambda}\left\lceil\Sigma_{0}\right.$ (for (1.3)) which are compactly supported in $B(0, R)$, such that

$$
\| \phi_{\lambda}\left\lceil\Sigma_{0}-\phi_{0}\left\lceil\Sigma_{0}-\sum_{\mathbf{A}} \lambda F_{\mathbf{A}} \cos \left(\frac{u_{\mathbf{A}}}{\lambda}\right)\left\lceil\Sigma_{0} \|_{H^{5}} \leq \sqrt{2} \varepsilon \lambda^{2},\right.\right.\right.
$$


- The following condition holds initially

$$
\| \dot{\phi}_{\lambda}\left\lceil\Sigma_{0}-\dot{\phi}_{0}\left\lceil\Sigma_{0}+\sum_{\mathbf{A}} e^{\gamma_{0}}\left|\nabla u_{\mathbf{A}}\right| F_{\mathbf{A}} \sin \left(\frac{u_{\mathbf{A}}}{\lambda}\right)\left\lceil\Sigma_{0} \|_{H^{4}} \leq \sqrt{2} \varepsilon \lambda^{2} ;\right.\right.\right.
$$

$$
\int_{\Sigma_{0}} \dot{\phi}_{\lambda} \partial_{j} \phi_{\lambda} d x=0
$$

Proof. We will construct $\phi_{\lambda}\left\lceil\Sigma_{0}\right.$ and $\dot{\phi}_{\lambda}\left\lceil\Sigma_{0}\right.$ of the following form:

$$
\begin{gathered}
\phi_{\lambda}\left\lceil\Sigma_{0}=\phi_{0}\left\lceil\Sigma_{0}+\sum_{\mathbf{A}} \lambda F_{\mathbf{A}} \cos \left(\frac{u_{\mathbf{A}}}{\lambda}\right)\left\lceil\Sigma_{0}+\Omega_{0} r_{0},\right.\right.\right. \\
\dot{\phi}_{\lambda}\left\lceil\Sigma_{0}=\dot{\phi}_{0}\left\lceil\Sigma_{0}-\sum_{\mathbf{A}} e^{\gamma_{0}}\left|\nabla u_{\mathbf{A}}\right| F_{\mathbf{A}} \sin \left(\frac{u_{\mathbf{A}}}{\lambda}\right)\left\lceil\Sigma_{0}+\Omega_{1} r_{1},\right.\right.\right.
\end{gathered}
$$

where $r_{0}, r_{1}: \mathbb{R}^{2} \rightarrow \mathbb{R}$ are smooth functions compactly supported in $B(0, R)$ independent of $\lambda$ to be chosen below, with

and $\Omega_{0}, \Omega_{1} \in \mathbb{R}$ (depending on $\lambda$ ).

$$
\left\|r_{0}\right\|_{H^{10}}+\left\|r_{1}\right\|_{H^{9}} \leq \varepsilon
$$

By the genericity assumption (4.1), there exists $r_{0}$ and $r_{1}$ (smooth and compactly supported in $B(0, R)$ ) such that for some $c_{p}>0$,

$$
\left|\operatorname{det}\left(\begin{array}{ll}
-\int_{\Sigma_{0}} r_{0} \partial_{1} \dot{\phi}_{0} d x & \int_{\Sigma_{0}} r_{1} \partial_{1} \phi_{0} d x \\
-\int_{\Sigma_{0}} r_{0} \partial_{2} \dot{\phi}_{0} d x & \int_{\Sigma_{0}} r_{1} \partial_{2} \phi_{0} d x
\end{array}\right)\right|>c_{p}>0
$$

This can for instance be achieved by taking $r_{0}$ and $r_{1}$ to be supported in a very small neighborhood of $p$ (as in (4.1)).

Without loss of generality, we can rescale the functions $r_{0}$ and $r_{1}$ so that (4.12) holds. Notice that we must have $c_{p} \lesssim \varepsilon^{4}$, but in general, it is possible that $c_{p} \ll \varepsilon$.

Our goal now is to find $\Omega_{0}$ and $\Omega_{1}$ (satisfying appropriate bounds) such that

This condition can be written as

$$
\int_{\Sigma_{0}} \dot{\phi}_{\lambda} \partial_{j} \phi_{\lambda} d x=0
$$

$$
\begin{aligned}
& 0=\int_{\Sigma_{0}} \underbrace{\Omega_{0} \partial_{j} r_{0} \dot{\phi}_{0}+\Omega_{1} r_{1} \partial_{j} \phi_{0}}_{=: \text {Main term }}+\underbrace{\dot{\phi}_{0} \partial_{j} \phi_{0}+\sum_{\mathbf{A}} e^{\gamma_{0}} F_{\mathbf{A}}^{2}\left|\nabla u_{\mathbf{A}}\right| \partial_{j} u_{\mathbf{A}} \sin ^{2}\left(\frac{u_{\mathbf{A}}}{\lambda}\right)}_{\text {Background terms }} \\
& +\int_{\Sigma_{0}} \underbrace{-\Omega_{0} \partial_{j} r_{0} \sum_{\mathbf{A}} e^{\gamma_{0}}\left|\nabla u_{\mathbf{A}}\right| F_{\mathbf{A}} \sin \left(\frac{u_{\mathbf{A}}}{\lambda}\right)}_{=: H F_{1}}+\underbrace{\Omega_{1} r_{1} \partial_{j}\left(\sum_{\mathbf{A}} \lambda F_{\mathbf{A}} \cos \left(\frac{u_{\mathbf{A}}}{\lambda}\right)\right)}_{=: H F_{2}} \\
& +\int_{\Sigma_{0}} \underbrace{-\partial_{j} \phi_{0} \sum_{\mathbf{A}} e^{\gamma_{0}}\left|\nabla u_{\mathbf{A}}\right| F_{\mathbf{A}} \sin \left(\frac{u_{\mathbf{A}}}{\lambda}\right)}_{=: H F_{3}}+\underbrace{\dot{\phi}_{0} \partial_{j}\left(\sum_{\mathbf{A}} \lambda F_{\mathbf{A}} \cos \left(\frac{u_{\mathbf{A}}}{\lambda}\right)\right)}_{=: H F_{4}} \\
& -\int_{\Sigma_{0}} \underbrace{\left(\sum_{\mathbf{A}} e^{\gamma_{0}}\left|\nabla u_{\mathbf{A}}\right| F_{\mathbf{A}} \sin \left(\frac{u_{\mathbf{A}}}{\lambda}\right)\right)\left(\sum_{\mathbf{B}} \lambda\left(\partial_{j} F_{\mathbf{B}}\right) \cos \left(\frac{u_{\mathbf{B}}}{\lambda}\right)\right)}_{=: H F_{5}} \\
& +\int_{\Sigma_{0}} \underbrace{\sum_{\mathbf{A}} \sum_{\mathbf{B} \neq \mathbf{A}} e^{\gamma_{0}} F_{\mathbf{A}} F_{\mathbf{B}}\left|\nabla u_{\mathbf{A}}\right| \partial_{j} u_{\mathbf{B}} \sin \left(\frac{u_{\mathbf{A}}}{\lambda}\right) \sin \left(\frac{u_{\mathbf{B}}}{\lambda}\right)}_{=: H F_{6}}+\underbrace{\Omega_{0} \Omega_{1} \partial_{j} r_{0} r_{1}}_{=: \text {Small term }} .
\end{aligned}
$$

We first consider the "background terms" above. We have

$$
\sum_{\mathbf{A}} e^{\gamma_{0}} F_{\mathbf{A}}^{2}\left|\nabla u_{\mathbf{A}}\right| \partial_{j} u_{\mathbf{A}} \sin ^{2}\left(\frac{u_{\mathbf{A}}}{\lambda}\right)=\frac{1}{2} \sum_{\mathbf{A}} \breve{F}_{\mathbf{A}}^{2}\left|\nabla u_{\mathbf{A}}\right| \partial_{j} u_{\mathbf{A}}\left(1+\cos \left(\frac{2 u_{\mathbf{A}}}{\lambda}\right)\right) .
$$

\footnotetext{
16 where for simplicity we have suppressed the measure $d x$.
} 
Let $v_{\mathbf{A}}=2 u_{\mathbf{A}}$. Since $\left\{u_{\mathbf{A}}\right\}_{\mathbf{A} \in \mathcal{A}}$ is spatially adapted (see Definition [4.6), we have $\left|\nabla u_{\mathbf{A}}\right|>\frac{1}{4}$ and consequently $\left|\nabla v_{\mathbf{A}}\right|>\frac{1}{2}$. Therefore, we can iteratively apply $\sin \left(\frac{v_{\mathbf{A}}}{\lambda}\right)=-\frac{\lambda^{2} \Delta\left(\sin \left(\frac{v_{\mathbf{A}}}{\lambda}\right)\right)}{\left|\nabla v_{\mathbf{A}}\right|^{2}}+\frac{\lambda\left(\Delta v_{\mathbf{A}}\right) \cos \left(\frac{v_{\mathbf{A}}}{\lambda}\right)}{\left|\nabla v_{\mathbf{A}}\right|^{2}}$ and $\cos \left(\frac{v_{\mathbf{A}}}{\lambda}\right)=-\frac{\lambda^{2} \Delta\left(\cos \left(\frac{v_{\mathbf{A}}}{\lambda}\right)\right)}{\left|\nabla v_{\mathbf{A}}\right|^{2}}-\frac{\lambda\left(\Delta v_{\mathbf{A}}\right) \sin \left(\frac{v_{\mathbf{A}}}{\lambda}\right)}{\left|\nabla v_{\mathbf{A}}\right|^{2}}$ and integrate by parts to obtain 17

$$
\int_{\Sigma_{0}} \breve{F}_{\mathbf{A}}^{2}\left|\nabla u_{\mathbf{A}}\right| \cos \left(\frac{2 u_{\mathbf{A}}}{\lambda}\right) d x=O\left(\varepsilon^{2} \lambda^{3}\right) .
$$

Notice that we indeed have (more than) sufficient regularity for the background to justify this calculation. By the hypothesis on the initial data for the background metric we have

$$
\int_{\Sigma_{0}}\left(2 \dot{\phi}_{0} \partial_{j} \phi_{0}+\sum \breve{F}_{\mathbf{A}}^{2}\left|\nabla u_{\mathbf{A}}\right| \partial_{j} u_{\mathbf{A}}\right) d x=0
$$

Therefore, we have

$$
\text { |"Background term" } \mid=O\left(\varepsilon^{2} \lambda^{3}\right) \text {. }
$$

Next, note that all the terms that we have denoted as "high frequency" (HF), i.e., $H F_{1}, H F_{2}, H F_{3}, H F_{4}$, $H F_{5}$ and $H F_{6}$, contain an oscillating factor $\sin \left(\frac{v}{\lambda}\right)$ or $\cos \left(\frac{v}{\lambda}\right)$ with $|\nabla v|>\frac{1}{4}$ since $\left\{u_{\mathbf{A}}\right\}_{\mathbf{A} \in \mathcal{A}}$ is spatially adapted. Here, we have used the simple trigonometric identities

$$
\sin \left(\frac{u_{\mathbf{A}}}{\lambda}\right) \sin \left(\frac{u_{\mathbf{B}}}{\lambda}\right)=\frac{1}{2} \sum_{ \pm}(\mp 1) \cos \left(\frac{u_{\mathbf{A}} \pm u_{\mathbf{B}}}{\lambda}\right), \quad \sin \left(\frac{u_{\mathbf{A}}}{\lambda}\right) \cos \left(\frac{u_{\mathbf{B}}}{\lambda}\right)=\frac{1}{2} \sum_{ \pm} \sin \left(\frac{u_{\mathbf{A}} \pm u_{\mathbf{B}}}{\lambda}\right) .
$$

Therefore, by the argument as above, we also have

$$
H F_{1}=O\left(\varepsilon^{2} \lambda^{3}\right) \Omega_{0}, \quad H F_{2}=O\left(\varepsilon^{2} \lambda^{3}\right) \Omega_{1} .
$$

and for $i=3,4,5,6$,

$$
\left|H F_{i}\right|=O\left(\varepsilon^{2} \lambda^{3}\right) .
$$

Therefore, to solve for (4.15) for $j=1,2$ is equivalent to finding $\Omega_{0}, \Omega_{1}$ solving the following problem

$$
\mathbf{A}\left(\begin{array}{c}
\Omega_{0} \\
\Omega_{1}
\end{array}\right)=\mathbf{a}+\mathbf{B}\left(\begin{array}{c}
\Omega_{0} \\
\Omega_{1}
\end{array}\right)+\Omega_{0} \Omega_{1} \mathbf{c}
$$

where $\mathbf{A}=\left(\begin{array}{cc}-\int_{\Sigma_{0}} r_{0} \partial_{1} \dot{\phi}_{0} d x & \int_{\Sigma_{0}} r_{1} \partial_{1} \phi_{0} d x \\ -\int_{\Sigma_{0}} r_{0} \partial_{2} \dot{\phi}_{0} d x & \int_{\Sigma_{0}} r_{1} \partial_{2} \phi_{0} d x\end{array}\right)$, a is a 2 D vector with $\|\mathbf{a}\|_{2} \lesssim \varepsilon^{2} \lambda^{3}, \mathbf{B}$ is a $2 \times 2$ matrix with $\|\mathbf{B}\|_{2} \lesssim \varepsilon^{2} \lambda^{3}$ and $\mathbf{c}$ is a $2 \mathrm{D}$ vector with $\|\mathbf{c}\|_{2} \lesssim \varepsilon^{2}$. Since the determinant is a continuous function, by choosing $\lambda$ sufficiently small (depending on $c_{p}$ ), $\mathbf{A}-\mathbf{B}$ is invertible. We can thus define the (continuous) map $\Phi: \mathbb{R}^{2} \rightarrow \mathbb{R}^{2}$ by

$$
\Phi\left(\begin{array}{c}
x_{0} \\
x_{1}
\end{array}\right)=(\mathbf{A}-\mathbf{B})^{-1}\left(\mathbf{a}+x_{0} x_{1} \mathbf{c}\right) .
$$

Choosing $\lambda$ sufficiently small (say, $\lambda^{\frac{1}{2}} \ll c_{p}^{-1}$ ), it is easy to verify that $\Phi$ maps the closed ball $\overline{B\left(0, \lambda^{2}\right)}$ to itself. Hence, by Brouwer's fixed point theorem, $\Phi$ has a fixed point $\left(\begin{array}{c}\Omega_{0} \\ \Omega_{1}\end{array}\right)$, which is a solution to (4.16) satisfying

$$
\left|\Omega_{0}\right|+\left|\Omega_{1}\right| \leq \sqrt{2} \sqrt{\Omega_{0}^{2}+\Omega_{1}^{2}} \leq \sqrt{2} \lambda^{2} .
$$

Therefore, we have now found $\phi_{\lambda}\left\lceil\Sigma_{0}\right.$ and $\dot{\phi}_{\lambda}\left\lceil\Sigma_{0}\right.$ such that (4.14) holds. Moreover, combining (4.10), (4.11), (4.12) and (4.17), it is easy to check that the required bounds for $\phi_{\lambda}\left\lceil\Sigma_{0}\right.$ and $\dot{\phi}_{\lambda}\left\lceil\Sigma_{0}\right.$ hold.

Remark 4.10. We note from the proof above that the choice of $\lambda_{0}$ not only depends on the initial $u_{\mathbf{A}} \backslash_{\Sigma_{0}}$, but also depends on the profile of the background solution. This is because the choice of $\lambda_{0}$ depends on $c_{p}$ in (4.13), which can be viewed as a more quantitative version of (4.1).

\footnotetext{
${ }^{17}$ Here, and below in this proof, the constant depends on the initial data for $u_{\mathbf{A}}$.
} 
4.4. Construction of the parametrix. By Theorem 3.3, the one-parameter family of free admissible initial data to (1.3) constructed in Lemma 4.9 gives rise to a unique one-parameter family of solutions in the elliptic gauge. To analyze the solution arising from data given in Lemma 4.9, we decompose the scalar field $\phi_{\lambda}$ and the metric $g_{\lambda}$. It will be convenient notationally to suppress the subscripts $\lambda$ when there is no danger of confusion. We will however keep the subscripts 0 for the background $\phi_{0}$ and $g_{0}$.

The definitions of the parametrix for $\phi_{\lambda}$ and $g_{\lambda}$ are coupled in the sense that their mains terms are defined not only in terms of the background solution, but are defined to satisfy a coupled system of PDEs. At this point, it is already useful to keep in mind that we will decompose $\phi_{\lambda}$ as

$$
\begin{aligned}
\phi_{\lambda}= & \phi_{0}+\sum_{\mathbf{A}} \lambda F_{\mathbf{A}} \cos \left(\frac{u_{\mathbf{A}}}{\lambda}\right)+\sum_{\mathbf{A}} \lambda^{2} \widetilde{F}_{\mathbf{A}} \sin \left(\frac{u_{\mathbf{A}}}{\lambda}\right) \\
& +\sum_{\mathbf{A}} \lambda^{2} \widetilde{F}_{\mathbf{A}}^{(2)} \cos \left(\frac{2 u_{\mathbf{A}}}{\lambda}\right)+\sum_{\mathbf{A}} \lambda^{2} \widetilde{F}_{\mathbf{A}}^{(3)} \sin \left(\frac{3 u_{\mathbf{A}}}{\lambda}\right)+\mathcal{E}_{\lambda}
\end{aligned}
$$

and decompose each metric component $\mathfrak{g}$ (i.e., $\left.\mathfrak{g} \in\left\{\gamma, N, \beta^{i}\right\}\right)$ as

$$
\mathfrak{g}=\mathfrak{g}_{0}+\mathfrak{g}_{1}+\mathfrak{g}_{2}+\mathfrak{g}_{3}
$$

where $\mathfrak{g}_{0}$ is the corresponding metric component of the background solution. We will define all of these terms below in Sections 4.4.1 and 4.4.2

4.4.1. Parametrix for $\phi_{\lambda}$. The parametrix for $\phi_{\lambda}$ is constructed as follows:

$$
\begin{aligned}
\phi_{\lambda}= & \phi_{0}+\sum_{\mathbf{A}} \lambda F_{\mathbf{A}} \cos \left(\frac{u_{\mathbf{A}}}{\lambda}\right)+\sum_{\mathbf{A}} \lambda^{2} \widetilde{F}_{\mathbf{A}} \sin \left(\frac{u_{\mathbf{A}}}{\lambda}\right) \\
& +\sum_{\mathbf{A}} \lambda^{2} \widetilde{F}_{\mathbf{A}}^{(2)} \cos \left(\frac{2 u_{\mathbf{A}}}{\lambda}\right)+\sum_{\mathbf{A}} \lambda^{2} \widetilde{F}_{\mathbf{A}}^{(3)} \sin \left(\frac{3 u_{\mathbf{A}}}{\lambda}\right)+\mathcal{E}_{\lambda} .
\end{aligned}
$$

Here, $\phi_{0}, F_{\mathbf{A}}$ and $u_{\mathbf{A}}$ are the background quantities; $\mathcal{E}_{\lambda}$ is the "error term 18 "; and $\widetilde{F}_{\mathbf{A}}, \widetilde{F}_{\mathbf{A}}^{(2)}$ and $\widetilde{F}_{\mathbf{A}}^{(3)}$ are defined to be solutions to the following transport equations with zero initial data:

$$
\begin{gathered}
2\left(g_{0}^{-1}\right)^{\alpha \beta} \partial_{\alpha} u_{\mathbf{A}} \partial_{\beta} \widetilde{F}_{\mathbf{A}}+\left(\square_{g_{0}} u_{\mathbf{A}}\right) \widetilde{F}_{\mathbf{A}}=\frac{1}{\lambda^{2}} F_{\mathbf{A}}\left(g_{3}^{\prime}\right)^{\alpha \beta} \partial_{\alpha} u_{\mathbf{A}} \partial_{\beta} u_{\mathbf{A}}-\square_{g_{0}} F_{\mathbf{A}}+A_{\mathbf{A}}^{(1)}, \\
2\left(g_{0}^{-1}\right)^{\alpha \beta} \partial_{\alpha} u_{\mathbf{A}} \partial_{\beta} \widetilde{F}_{\mathbf{A}}^{(2)}+\left(\square_{g_{0}} u_{\mathbf{A}}\right) \widetilde{F}_{\mathbf{A}}^{(2)}=A_{\mathbf{A}}^{(2)}, \\
2\left(g_{0}^{-1}\right)^{\alpha \beta} \partial_{\alpha} u_{\mathbf{A}} \partial_{\beta} \widetilde{F}_{\mathbf{A}}^{(3)}+\left(\square_{g_{0}} u_{\mathbf{A}}\right) \widetilde{F}_{\mathbf{A}}^{(3)}=A_{\mathbf{A}}^{(3)},
\end{gathered}
$$

where

- $A_{\mathbf{A}}^{(1)}, A_{\mathbf{A}}^{(2)}$ and $A_{\mathbf{A}}^{(3)}$ are compactly supported expressions which depend only on the background (and can be explicitly written down 19 ) and obey the bounds

$$
\left\|A_{\mathbf{A}}^{(a)}\right\|_{H^{8} \cap C^{8}}+\left\|\partial_{t} A_{\mathbf{A}}^{(a)}\right\|_{H^{7} \cap C^{7}} \leq C\left(C_{0}\right) \varepsilon^{3} \quad \text { for } a=1,2,3
$$

and

- for $a=1,3$, we define

$$
\begin{aligned}
g_{a}^{\prime}= & \left(\begin{array}{ccc}
\frac{2 N_{a}}{N_{0}^{3}} & 0 & 0 \\
0 & e^{-2 \gamma_{0}}\left(-1+e^{-2 \gamma_{a}}\right) & 0 \\
0 & 0 & e^{-2 \gamma_{0}}\left(-1+e^{-2 \gamma_{a}}\right)
\end{array}\right) \\
& -\frac{2 N_{a}}{N_{0}^{3}}\left(\begin{array}{ccc}
0 & \beta_{0}^{1} & \beta_{0}^{2} \\
\left(\beta_{0}\right)^{1} & -\left(\beta_{0}\right)^{1}\left(\beta_{0}\right)^{1} & -\left(\beta_{0}\right)^{1}\left(\beta_{0}\right)^{2} \\
\left(\beta_{0}\right)^{2} & -\left(\beta_{0}\right)^{1}\left(\beta_{0}\right)^{2} & -\left(\beta_{0}\right)^{2}\left(\beta_{0}\right)^{2}
\end{array}\right) \\
& +\frac{1}{N_{0}^{2}}\left(\begin{array}{ccc}
0 & \left(\beta_{a}\right)^{1} & \left(\beta_{a}\right)^{2} \\
\left(\beta_{a}\right)^{1} & -2\left(\beta_{0}\right)^{1}\left(\beta_{a}\right)^{1} & -\left(\beta_{0}\right)^{1}\left(\beta_{a}\right)^{2}-\left(\beta_{a}\right)^{1}\left(\beta_{0}\right)^{2} \\
\left(\beta_{a}\right)^{2} & -\left(\beta_{0}\right)^{1}\left(\beta_{a}\right)^{2}-\left(\beta_{a}\right)^{1}\left(\beta_{0}\right)^{2} & -2\left(\beta_{0}\right)^{2}\left(\beta_{a}\right)^{2}
\end{array}\right) .
\end{aligned}
$$

\footnotetext{
${ }^{18}$ As we will see later in Section 6.5 we will further decompose $\mathcal{E}_{\lambda}$ into extra terms and some of which can be viewed as "main terms" of $\mathcal{E}_{\lambda}$. Nevertheless, at this point of the discussion, it suffices to note that each of these decomposed terms of $\mathcal{E}_{\lambda}$ are indeed "smaller" than the terms $\phi_{0}, \sum_{\mathbf{A}} \lambda F_{\mathbf{A}} \cos \left(\frac{u_{\mathbf{A}}}{\lambda}\right), \sum_{\mathbf{A}} \lambda^{2} \widetilde{F}_{\mathbf{A}} \sin \left(\frac{u_{\mathbf{A}}}{\lambda}\right), \sum_{\mathbf{A}} \lambda^{2} \widetilde{F}_{\mathbf{A}}^{(2)} \cos \left(\frac{2 u_{\mathbf{A}}}{\lambda}\right)$ and $\sum_{\mathbf{A}} \lambda^{2} \widetilde{F}_{\mathbf{A}}^{(3)} \sin \left(\frac{3 u_{\mathbf{A}}}{\lambda}\right)$

${ }^{19}$ We however will not do that as the explicit expressions are rather tedious.
} 
We will not define until Section 6.2 the expressions $A_{\mathbf{A}}^{(1)}, A_{\mathbf{A}}^{(2)}$ and $A_{\mathbf{A}}^{(3)}$ (see Definition 6.8). They are of course chosen so that we indeed have a good parametrix, i.e., so that the main terms in the expression $\square_{g} \phi_{\lambda}$ cancel.

We remark that $g_{a}^{\prime}$ is constructed as follows: Let $g_{0}+g_{a}$ be the matrix with components given by $\mathfrak{g}_{0}+\mathfrak{g}_{a}$. Then $g_{a}^{\prime}$ is the linear in $\mathfrak{g}_{a}$ term of the expression $\left(g_{0}+g_{a}\right)^{-1}-\left(g_{0}\right)^{-1}$.

Here, two important remarks are in order:

- In the definition above, $F_{\mathbf{A}}$ and $u_{\mathbf{A}}$ manifestly depend only on the background. The functions $\widetilde{F}_{\mathbf{A}}^{(2)}$ and $\widetilde{F}_{\mathbf{A}}^{(3)}$ are also chosen to depend only on the background. Therefore, the only part that interacts with the metric $g_{\lambda}$ are $\widetilde{F}_{\mathbf{A}}$ and the error term $\mathcal{E}_{\lambda}$.

- The parametrix as defined above captures up to order $O_{\varepsilon}\left(\lambda^{2}\right)$ of terms that are oscillating along a null direction of the background. The d'Alembertian of these terms is a $O_{\varepsilon}(\lambda)$. The multilinear interactions also give rise to terms of order $O_{\varepsilon}(\lambda)$ with oscillating factors such as $\sin \left(\frac{u_{\mathrm{A}} \pm u_{\mathrm{B}}}{\lambda}\right)$ (with $\mathbf{A} \neq \mathbf{B})$. Since the $\left\{u_{\mathbf{A}}\right\}$ are null adapted, these terms oscillate in a non-null direction and therefore behave better. Hence, we treat these terms as part of the error term.

4.4.2. Parametrix for $g_{\lambda}$. For the metric $g_{\lambda}$, we construct a parametrix for each metric component. More precisely, we will define

$$
N=N_{0}+N_{1}+N_{2}+N_{3}, \quad \gamma=\gamma_{0}+\gamma_{1}+\gamma_{2}+\gamma_{3}, \quad \beta^{i}=\left(\beta^{i}\right)_{0}+\left(\beta^{i}\right)_{1}+\left(\beta^{i}\right)_{2}+\left(\beta^{i}\right)_{3} .
$$

We will use the convention that $\mathfrak{g}$ denotes one of these metric components. In this notation, the decomposition above reads

$$
\mathfrak{g}=\mathfrak{g}_{0}+\mathfrak{g}_{1}+\mathfrak{g}_{2}+\mathfrak{g}_{3}
$$

In order to treat these metric components in a unified manner, we consider the Poisson-type equations satisfied by them. We consider the equations (3.8)-(3.11) (in the vacuum case, i.e., when $F_{\mathbf{A}}=0$ ). Taking the divergence of (3.11), using (3.8) and eliminating $H$ (using (3.11)), we thus obtain the following system of elliptic equations for each of the metric components

$$
\begin{aligned}
& \Delta \gamma=-|\nabla \phi|^{2}-\frac{e^{2 \gamma}}{N^{2}}\left(e_{0} \phi\right)^{2}-\frac{e^{2 \gamma}}{8 N^{2}}|L \beta|^{2}, \\
& \Delta N=\frac{e^{2 \gamma}}{4 N}|L \beta|^{2}+\frac{2 e^{2 \gamma}}{N}\left(e_{0} \phi\right)^{2}, \\
& \Delta \beta^{j}=\delta^{i k} \delta^{j \ell} \partial_{k}\left(\log \left(N e^{-2 \gamma}\right)\right)(L \beta)_{i \ell}-4 \delta^{i j}\left(e_{0} \phi\right)\left(\partial_{i} \phi\right) .
\end{aligned}
$$

We now introduce some notations which will allow us to deal with (4.25), (4.26) and (4.27) simultaneously.

For $\gamma, N, \beta^{i}$, define the following matrices. Notice that these correspond to the quadratic terms in $\phi$ in (4.25)-(4.27) (cf. (4.35) ):

$$
\begin{aligned}
\boldsymbol{\Gamma}(\gamma)^{\mu \nu} & =\left(\begin{array}{ccc}
-\frac{e^{2 \gamma}}{N^{2}} & \frac{e^{2 \gamma}}{N^{2}} \beta^{1} & \frac{e^{2 \gamma}}{N^{2}} \beta^{2} \\
\frac{e^{2 \gamma}}{N^{2}} \beta^{1} & -1-\frac{e^{2 \gamma}}{N^{2}}\left(\beta^{1}\right)^{2} & -\frac{e^{2 \gamma}}{N^{2}} \beta^{1} \beta^{2} \\
\frac{e^{2 \gamma}}{N^{2}} \beta^{2} & -\frac{e^{2 \gamma}}{N^{2}} \beta^{1} \beta^{2} & -1-\frac{e^{2 \gamma}}{N^{2}}\left(\beta^{2}\right)^{2}
\end{array}\right), \\
\boldsymbol{\Gamma}(N)^{\mu \nu} & =\left(\begin{array}{ccc}
-\frac{2 e^{2 \gamma}}{N} & \frac{2 e^{2 \gamma}}{N} \beta^{1} & \frac{2 e^{2 \gamma}}{N} \beta^{2} \\
\frac{2 e^{2 \gamma}}{N} \beta^{1} & -\frac{2 e^{2 \gamma}}{N}\left(\beta^{1}\right)^{2} & -\frac{2 e^{2 \gamma}}{N} \beta^{1} \beta^{2} \\
\frac{2 e^{2 \gamma}}{N} \beta^{2} & -\frac{2 e^{2 \gamma}}{N} \beta^{1} \beta^{2} & -\frac{2 e^{2 \gamma}}{N}\left(\beta^{2}\right)^{2}
\end{array}\right), \\
\boldsymbol{\Gamma}\left(\beta^{1}\right)^{\mu \nu} & =\left(\begin{array}{ccc}
0 & -2 & 0 \\
-2 & 4 \beta^{1} & 2 \beta^{2} \\
0 & 2 \beta^{2} & -0
\end{array}\right), \\
\boldsymbol{\Gamma}\left(\beta^{2}\right)^{\mu \nu} & =\left(\begin{array}{ccc}
0 & 0 & -2 \\
0 & 0 & 2 \beta^{1} \\
-2 & 2 \beta^{1} & 4 \beta^{2}
\end{array}\right) .
\end{aligned}
$$

Define also the matrices $\boldsymbol{\Gamma}_{0}(\gamma), \boldsymbol{\Gamma}_{0}(N)$ and $\boldsymbol{\Gamma}_{0}\left(\beta^{i}\right)$ in a similar manner as above except that all of the metric components $\gamma, N$ and $\beta^{i}$ are replaced by their background value $\gamma_{0}, N_{0}$ and $\beta_{0}^{i}$.

\footnotetext{
$20_{\text {i.e., terms that }}$ are $\sim \lambda^{2}$ with a constant depending on $\varepsilon$.
} 
Next, define the following functions

$$
\begin{aligned}
\Upsilon(\gamma) & :=-\frac{e^{2 \gamma}}{8 N^{2}}|L \beta|^{2}, \\
\Upsilon(N) & :=\frac{e^{2 \gamma}}{4 N}|L \beta|^{2}, \\
\Upsilon\left(\beta^{i}\right) & :=\delta^{j k} \delta^{i \ell}\left(\left(\partial_{k} \log N\right)(L \beta)_{j \ell}-2\left(\partial_{k} \gamma\right)(L \beta)_{j \ell}\right) .
\end{aligned}
$$

Similarly as above, we use $\Upsilon_{0}(\gamma), \Upsilon_{0}(N)$ and $\Upsilon_{0}\left(\beta^{i}\right)$ to denote the above expressions when all of the metric components $\gamma, N$ and $\beta^{i}$ are replaced by their background value $\gamma_{0}, N_{0}$ and $\beta_{0}^{i}$ (including both the ones that are differentiated and those that are not).

Therefore, by the above notations, (4.25), (4.26) and (4.27) now take the form

$$
\Delta \mathfrak{g}=\boldsymbol{\Gamma}(\mathfrak{g})^{\mu \nu} \partial_{\mu} \phi \partial_{\nu} \phi+\Upsilon(\mathfrak{g}) .
$$

The background metric components, which are denoted by $\mathfrak{g}_{0}$, satisfies a similar equation with an extra term

$$
\Delta \mathfrak{g}_{0}=\boldsymbol{\Gamma}_{0}(\mathfrak{g})^{\mu \nu} \partial_{\mu} \phi_{0} \partial_{\nu} \phi_{0}+\frac{1}{2} \sum_{\mathbf{A}} F_{\mathbf{A}}^{2} \boldsymbol{\Gamma}_{0}(\mathfrak{g})^{\mu \nu}\left(\partial_{\mu} u_{\mathbf{A}}\right)\left(\partial_{\nu} u_{\mathbf{A}}\right)+\Upsilon_{0}(\mathfrak{g}) .
$$

Definition of $\mathfrak{g}_{1}$

Define $\mathfrak{g}_{1}$ by

$$
\begin{aligned}
\mathfrak{g}_{1}= & -\frac{1}{8} \boldsymbol{\Gamma}_{0}(\mathfrak{g})^{\mu \nu} \sum_{\mathbf{A}} \frac{\lambda^{2} F_{\mathbf{A}}^{2}}{\left|\nabla u_{\mathbf{A}}\right|^{2}}\left(\partial_{\mu} u_{\mathbf{A}}\right)\left(\partial_{\nu} u_{\mathbf{A}}\right) \cos \left(\frac{2 u_{\mathbf{A}}}{\lambda}\right) \\
& -2 \boldsymbol{\Gamma}_{0}(\mathfrak{g})^{\mu \nu} \sum_{\mathbf{A}} \frac{\lambda^{2} F_{\mathbf{A}}}{\left|\nabla u_{\mathbf{A}}\right|^{2}}\left(\partial_{\mu} \phi_{0}\right)\left(\partial_{\nu} u_{\mathbf{A}}\right) \sin \left(\frac{u_{\mathbf{A}}}{\lambda}\right) \\
& -\frac{1}{2} \boldsymbol{\Gamma}_{0}(\mathfrak{g})^{\mu \nu} \sum_{ \pm} \sum_{\mathbf{A}} \sum_{\mathbf{B} \neq \mathbf{A}} \frac{(\mp 1) \cdot \lambda^{2} F_{\mathbf{A}} F_{\mathbf{B}}}{\mid \nabla\left(u_{\mathbf{A}} \pm\left. u_{\mathbf{B}}\right|^{2}\right.}\left(\partial_{\mu} u_{\mathbf{A}}\right)\left(\partial_{\nu} u_{\mathbf{B}}\right) \cos \left(\frac{u_{\mathbf{A}} \pm u_{\mathbf{B}}}{\lambda}\right) .
\end{aligned}
$$

Recall that the (background) collection $\left\{u_{\mathbf{A}}\right\}_{\mathbf{A} \in \mathcal{A}}$ is spatially adapted and therefore the above expression is well-defined and bounded. Notice that the definition of $\mathfrak{g}_{1}$ depends only on the background. One can think of $\mathfrak{g}_{1}$ as the part of the parametrix that is constructed to cancel all the $O_{\varepsilon}(1) \overline{\text { high-frequency terms }}$ in $\Delta\left(\mathfrak{g}-\mathfrak{g}_{0}\right)$.

\section{Definition of $\mathfrak{g}_{2}$}

Define $\mathfrak{g}_{2}$ by

$$
\begin{aligned}
\mathfrak{g}_{2}:= & -\sum_{\mathbf{A}} \frac{\lambda^{3} \mathcal{G}_{1,1, \mathbf{A}}(\mathfrak{g})}{\left|\nabla u_{\mathbf{A}}\right|^{2}} \cos \left(\frac{u_{\mathbf{A}}}{\lambda}\right)-\sum_{\mathbf{A}} \frac{\lambda^{3} \mathcal{G}_{1,2, \mathbf{A}}(\mathfrak{g})}{4\left|\nabla u_{\mathbf{A}}\right|^{2}} \sin \left(\frac{2 u_{\mathbf{A}}}{\lambda}\right) \\
& -\sum_{\mathbf{A}} \frac{\lambda^{3} \mathcal{G}_{1,3, \mathbf{A}}(\mathfrak{g})}{9\left|\nabla u_{\mathbf{A}}\right|^{2}} \cos \left(\frac{3 u_{\mathbf{A}}}{\lambda}\right)-\sum_{ \pm} \sum_{\mathbf{A}} \sum_{\mathbf{B} \neq \mathbf{A}} \frac{\lambda^{3} \mathcal{G}_{2,1, \mathbf{A}, \mathbf{B}, \pm}(\mathfrak{g})}{\left|\nabla\left(u_{\mathbf{A}} \pm u_{\mathbf{B}}\right)\right|^{2}} \sin \left(\frac{u_{\mathbf{A}} \pm u_{\mathbf{B}}}{\lambda}\right) \\
& -\sum_{ \pm} \sum_{\mathbf{A}} \sum_{\mathbf{B} \neq \mathbf{A}} \frac{\lambda^{3} \mathcal{G}_{2,2, \mathbf{A}, \mathbf{B}, \pm}(\mathfrak{g})}{\left|\nabla\left(u_{\mathbf{A}} \pm 2 u_{\mathbf{B}}\right)\right|^{2}} \cos \left(\frac{u_{\mathbf{A}} \pm 2 u_{\mathbf{B}}}{\lambda}\right) \\
& -\sum_{ \pm} \sum_{\mathbf{A}, \mathbf{B}} \frac{\lambda^{3} \mathcal{G}_{2,3, \mathbf{A}, \mathbf{B}, \pm}(\mathfrak{g})}{\left|\nabla\left(u_{\mathbf{A}} \pm 3 u_{\mathbf{B}}\right)\right|^{2}} \sin \left(\frac{u_{\mathbf{A}} \pm 3 u_{\mathbf{B}}}{\lambda}\right),
\end{aligned}
$$

where for each $\mathfrak{g}$, the functions $\mathcal{G}_{1,1, \mathbf{A}}(\mathfrak{g}), \mathcal{G}_{1,2, \mathbf{A}}(\mathfrak{g}), \mathcal{G}_{1,3, \mathbf{A}}(\mathfrak{g}), \mathcal{G}_{2,1, \mathbf{A}, \mathbf{B}, \pm}(\mathfrak{g}), \mathcal{G}_{2,2, \mathbf{A}, \mathbf{B}, \pm}(\mathfrak{g})$ and $\mathcal{G}_{2,3, \mathbf{A}, \mathbf{B}, \pm}(\mathfrak{g})$ are all compactly supported. All these functions will be defined precisely in Section 7.2 and can in principle be written down explicitly. At this point, let us only note that these functions not only depend on the background solution, but also depends of $\widetilde{F}_{\mathbf{A}}$ defined earlier in (4.19). One can think of $\mathfrak{g}_{2}$ as the part of the parametrix that is constructed to cancel all the $O_{\varepsilon}(\lambda)$ high-frequency terms in $\Delta\left(\mathfrak{g}-\mathfrak{g}_{0}-\mathfrak{g}_{1}\right)$.

\section{Definition of $\mathfrak{g}_{3}$}

Finally, of course the term $\mathfrak{g}_{3}$ is determined by the evolution equation as well as (4.24).

\footnotetext{
${ }^{21}$ i.e., these terms may be small in terms of $\varepsilon$ but are not small in terms of $\lambda$.
} 


\section{The BOOTSTRAP ASSUMPtions}

In this section, we will describe all the bootstrap assumptions. Our goal will be to use a bootstrap argument to prove that for $\varepsilon$ and $\lambda$ sufficiently small (with $\lambda \ll \varepsilon$, consistent with Theorem 4.2) the solution arising from initial data given in Lemma 4.9 exists for time $[0,1]$ and that the parametrix we introduced in Section 4.4 is indeed a good approximation of the solution. This then also allows us to show convergence as $\lambda \rightarrow 0$ (cf. Section 8). We will use $C_{1}$ as a bootstrap constant, which will be determined later and will depend only on $C_{0}$, which is given by Corollary 3.5 . Our goal will be to improve all the bounds made in the bootstrap assumption. In order to emphasize which constants depend on $C_{1}$, we will use $C\left(C_{0}\right)$ to denote a constant depending only on $C_{0}$ and $C\left(C_{1}\right)$ to denote a constant depending on both $C_{0}$ and $C_{1}$. We will also use $C$ (or $\lesssim$ ) for constants which are independent of both $C_{0}$ and $C_{1}$.

Recall that in Section 4.4 we have introduced the decomposition of $\phi_{\lambda}$ and $g_{\lambda}$ into various pieces. We will therefore need to obtain estimates for all of them. Notice however that $\phi_{0}, F_{\mathbf{A}}, \widetilde{F}_{\mathbf{A}}^{(2)}, \widetilde{F}_{\mathbf{A}}^{(3)}, \mathfrak{g}_{0}$ and $\mathfrak{g}_{1}$ are defined in a way that only depend on the background solution. We therefore do not need bootstrap assumptions on them. Nevertheless, for $\widetilde{F}_{\mathbf{A}}^{(2)}, \overline{\widetilde{F}_{\mathbf{A}}^{(3)} \text { and } \mathfrak{g}_{1}}$, it is convenient to already state the bounds that they satisfy 22. Notice that all these bounds depend only on $C_{0}$ and are independent of the bootstrap constant.

$$
\begin{array}{r}
\left\|\widetilde{F}_{\mathbf{A}}^{(a)}\right\|_{H^{9}}+\left\|\partial_{t} \widetilde{F}_{\mathbf{A}}^{(a)}\right\|_{H^{8}}+\left\|\partial_{t}^{2} \widetilde{F}_{\mathbf{A}}^{(a)}\right\|_{H^{7}} \leq C\left(C_{0}\right) \varepsilon^{2}, \quad a=2,3, \\
\sum_{k \leq 8} \lambda^{k}\left\|\mathfrak{g}_{1}\right\|_{H^{k} \cap C^{k}}+\sum_{k \leq 7} \lambda^{k+1}\left\|\partial_{t} \mathfrak{g}_{1}\right\|_{H^{k} \cap C^{k}}+\sum_{k \leq 6} \lambda^{k+2}\left\|\partial_{t}^{2} \mathfrak{g}_{1}\right\|_{H^{k} \cap C^{k}} \leq C\left(C_{0}\right) \lambda^{2} \varepsilon^{2} .
\end{array}
$$

(B1) and (B2) will be proven in Propositions 6.16 and 7.10 respectively.

In view of the above discussions, we only need to introduce bootstrap assumptions for $\widetilde{F}_{\mathbf{A}}, \mathcal{E}_{\lambda}, \mathfrak{g}_{2}$ and $\mathfrak{g}_{3}$. Introduce the following bootstrap assumptions: 23 :

$$
\begin{aligned}
& \sum_{k \leq 3}\left(\lambda^{k}\left\|\widetilde{F}_{\mathbf{A}}\right\|_{H^{2+k}}+\lambda^{k}\left\|\partial_{t} \widetilde{F}_{\mathbf{A}}\right\|_{H^{1+k}}+\lambda^{k+1}\left\|\partial_{t}^{2} \widetilde{F}_{\mathbf{A}}\right\|_{H^{k}}\right) \leq C_{1} \varepsilon, \\
& \sum_{k \leq 3} \lambda^{k}\left\|\partial \mathcal{E}_{\lambda}\right\|_{H^{k}}+\sum_{k \leq 2} \lambda^{k+1}\left\|\partial^{2} \mathcal{E}_{\lambda}\right\|_{H^{k}} \leq C_{1} \varepsilon \lambda^{2}, \\
& \sum_{k \leq 5} \lambda^{k}\left\|\mathfrak{g}_{2}\right\|_{H^{k}}+\sum_{k \leq 4} \lambda^{k+1}\left\|\partial_{t} \mathfrak{g}_{2}\right\|_{H^{k}} \leq C_{1} \varepsilon \lambda^{3} .
\end{aligned}
$$

For $\mathfrak{g}_{3}$, each of the metric component can be decomposed as

$$
\mathfrak{g}_{3}=\left(\mathfrak{g}_{3}\right)_{\text {asymp }}(t) \chi(|x|) \log (|x|)+\widetilde{\mathfrak{g}}_{3},
$$

where

$$
\left|\left(\mathfrak{g}_{3}\right)_{\text {asymp }}\right|+\left|\partial_{t}\left(\mathfrak{g}_{3}\right)_{\text {asymp }}\right|+\sum_{k \leq 3} \lambda^{k}\left\|\widetilde{\mathfrak{g}}_{3}\right\|_{H_{\delta}^{2+k}}+\sum_{k \leq 2} \lambda^{k+1}\left\|\partial_{t} \widetilde{\mathfrak{g}}_{3}\right\|_{H_{\delta}^{2+k}} \leq C_{1} \varepsilon \lambda^{2} .
$$

The existence of such a decomposition is a consequence of the local existence result (Theorem 3.3). Notice that it also implies the following conditions for the full metric,

$$
\begin{gathered}
\mathfrak{g}=\left(\mathfrak{g}_{0}\right)_{c}+\left(\left(\mathfrak{g}_{0}\right)_{\text {asymp }}(t)+\left(\mathfrak{g}_{3}\right)_{\text {asymp }}(t)\right) \chi(|x|) \log (|x|)+\widetilde{\mathfrak{g}}_{0}+\mathfrak{g}_{1}+\mathfrak{g}_{2}+\widetilde{\mathfrak{g}}_{3} \\
\left(\mathfrak{g}_{0}\right)_{c}= \begin{cases}1 & \text { if } \mathfrak{g}=N \\
0 & \text { if } \mathfrak{g}=\gamma, \beta^{i},\end{cases}
\end{gathered}
$$

and

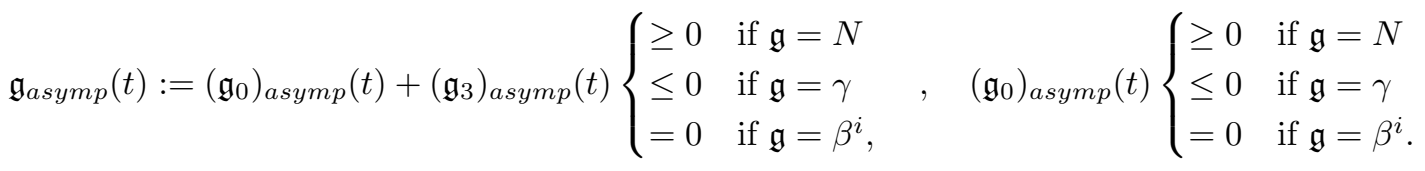

\footnotetext{
${ }^{22}$ We will however not restate the bounds for $\phi_{0}, F_{\mathbf{A}}$ and $\mathfrak{g}_{0}$ but will simply refer the readers to Corollary 3.5

${ }^{23}$ We will later decompose $\mathcal{E}_{\lambda}$ into three different pieces (see Section 6.5 and (6.19)). We will need the bootstrap assumption BA2 to hold for each of these pieces. We do not write this out explicitly at this point to simplify notations, but it will be clear in Section 6.5 that we indeed improve the bootstrap assumptions for each of those decomposed pieces.
} 
At this point it is useful to note the following support properties, which follows immediately from the estimates for the metric in Corollary 3.5, (B2), (BA3), (BA4) and the support statement in Theorem 3.3

Lemma 5.1. For $\varepsilon$ and $\lambda$ sufficiently small, as long as the bootstrap assumptions (BA3) and (BA4) hold on $[0, T](T \leq 1)$, there exists $R_{\text {supp }}>R$ (independent of $\lambda$ and $T$ ) such that

$$
J_{0}^{+}\left(\Sigma_{0} \cap B(0, R)\right), J_{\lambda}^{+}\left(\Sigma_{0} \cap B(0, R)\right) \subset[0, T] \times B\left(0, R_{\text {supp }}\right) .
$$

In particular, $\phi_{0}$ and $F_{\mathbf{A}}$ associated to the background solution to (1.4), as well as $\phi_{\lambda}$ associated to the one-parameter family of solutions to (1.3), are all supported in $B\left(0, R_{\text {supp }}\right)$ for all $t \in[0, T]$.

In fact, we will choose $A_{\mathbf{A}}^{(1)}, A_{\mathbf{A}}^{(2)}$ and $A_{\mathbf{A}}^{(3)}$ (cf. (4.19), (4.20), (4.21)) and $\mathcal{G}_{1,1, \mathbf{A}}(\mathfrak{g}), \mathcal{G}_{1,2, \mathbf{A}}(\mathfrak{g}), \mathcal{G}_{1,3, \mathbf{A}}(\mathfrak{g})$, $\mathcal{G}_{2,1, \mathbf{A}, \mathbf{B}, \pm}(\mathfrak{g}), \mathcal{G}_{2,2, \mathbf{A}, \mathbf{B}, \pm}(\mathfrak{g})$ and $\mathcal{G}_{2,3, \mathbf{A}, \mathbf{B}, \pm}(\mathfrak{g})$ (cf. (4.38) $)$ so that they are all supported in $J_{0}^{+}\left(\Sigma_{0} \cap B(0, R)\right)$. Hence, by (4.18), (4.19), (4.20), (4.21), (4.37), (4.38),

$$
\operatorname{supp}\left(F_{\mathbf{A}}\right), \operatorname{supp}\left(\widetilde{F}_{\mathbf{A}}\right), \operatorname{supp}\left(\widetilde{F}_{\mathbf{A}}^{(2)}\right), \operatorname{supp}\left(\widetilde{F}_{\mathbf{A}}^{(3)}\right), \operatorname{supp}\left(\mathcal{E}_{\lambda}\right), \operatorname{supp}\left(\mathfrak{g}_{1}\right), \operatorname{supp}\left(\mathfrak{g}_{2}\right) \subset B\left(0, R_{\text {supp }}\right),
$$

as long as the bootstrap assumption holds.

Finally, we need to introduce one more bootstrap assumption. Notice that the above bootstrap assumptions distinguish between the spatial $\nabla$ and the $\partial_{t}$ derivative. On the other hand, it will be crucial to our argument that $\partial_{t} \gamma_{3}$ is in fact better that the $\partial_{t}$ derivative of a general $\mathfrak{g}_{3}$ component. Namely, we make the following bootstrap assumption for $\partial_{t} \widetilde{\gamma}_{3}$ on the compact set $B\left(0, R_{\text {supp }}+1\right.$ ) (where $R_{\text {supp }}$ is as in Lemma 5.1):

$$
\left\|\partial_{t} \widetilde{\gamma}_{3}\right\|_{L^{2}\left(B\left(0, R_{\text {supp }}+1\right)\right)} \leq C_{1} \varepsilon \lambda^{2}
$$

Our goal will be to improve all the constants in (BA1), (BA2), (BA3), (BA4) and (BA5). The proof of which will occupy the rest of the paper. More precisely, we will prove

Theorem 5.2 (Bootstrap theorem). Let $\varepsilon$ and $\lambda$ be sufficiently small (with $\lambda \ll \varepsilon$ ) and $C_{1}$ be sufficiently large (depending on $C_{0}$ but independent of $\varepsilon$ and $\lambda$ ). Suppose that the bootstrap assumptions (BA1)-(BA5) hold for $t \in[0, T]$ for the unique solution arising from the initial data given in Lemma [4.9. Then in fact all of (BA1) -(BA5) hold for $t \in[0, T]$ with the constant $C_{1}$ replaced by $\frac{C_{1}}{2}$.

In view of Theorem 5.2, we will assume below that (BA1)-BA5 hold and that we have the following hierarchy of constants:

$$
\lambda \ll \varepsilon \ll C_{0} \ll C_{1} .
$$

In particular, we will freely take $C\left(C_{1}\right) \varepsilon<10^{-2}, C\left(C_{1}\right) \lambda<10^{-2}$ for constants $C\left(C_{1}\right)$ depending on $C_{1}$.

Before we end this section, let us note that our bootstrap assumptions imply immediate the following pointwise estimates via the Sobolev embbeding theorems in Appendix $\mathrm{A}$

Proposition 5.3. Under the bootstrap assumptions, the following estimates hold (for some $C\left(C_{0}\right)$ depending on $C_{0}$ and some universal constant $C$ ):

$$
\begin{gathered}
\sum_{k \leq 3} \lambda^{k}\left\|\widetilde{F}_{\mathbf{A}}\right\|_{C^{k}}+\sum_{k \leq 2} \lambda^{k+\frac{1}{2}}\left\|\partial_{t} \widetilde{F}_{\mathbf{A}}\right\|_{C^{k}}+\sum_{k \leq 1} \lambda^{k+2}\left\|\partial_{t}^{2} \widetilde{F}_{\mathbf{A}}\right\|_{C^{k}} \leq C C_{1} \varepsilon \\
\left(\left\|\widetilde{F}_{\mathbf{A}}^{(a)}\right\|_{H^{7}}+\left\|\partial_{t} \widetilde{F}_{\mathbf{A}}^{(a)}\right\|_{H^{6}}+\left\|\partial_{t}^{2} \widetilde{F}_{\mathbf{A}}^{(a)}\right\|_{H^{5}}\right) \leq C\left(C_{0}\right) \varepsilon^{2}, \quad a=2,3, \\
\sum_{k \leq 1} \lambda^{k}\left\|\partial \mathcal{E}_{\lambda}\right\|_{C^{k}}+\lambda\left\|\partial^{2} \mathcal{E}_{\lambda}\right\|_{C^{0}} \leq C C_{1} \varepsilon \lambda \\
\sum_{k \leq 3} \lambda^{k}\left\|\mathfrak{g}_{2}\right\|_{C^{k}}+\sum_{k \leq 2} \lambda^{k+1}\left\|\partial_{t} \mathfrak{g}_{2}\right\|_{C^{k}} \leq C C_{1} \varepsilon \lambda^{2} \\
\sum_{k \leq 3} \lambda^{k}\left\|\widetilde{\mathfrak{g}}_{3}\right\|_{C_{\delta+1}^{k}}+\sum_{k \leq 2} \lambda^{k+1}\left\|\partial_{t} \widetilde{\mathfrak{g}}_{3}\right\|_{C_{\delta+1}^{k}} \leq C C_{1} \varepsilon \lambda^{2}
\end{gathered}
$$

Proof. Estimates for the last two terms in (5.4), (5.6) and (5.7) follow from (BA1), (BA2), (BA3) and Proposition A.5. while estimates for the first term in (5.4), as well as (5.5) and (5.8), follow from (B1), (BA1), (BA4) and Proposition A.4 (in the particular case $H^{2+k} \subset C^{k}$ ).

\footnotetext{
${ }^{24}$ Here, $J_{0}^{+}$is the causal future with respect to the metric $g_{0}$ and $J_{\lambda}^{+}$is the causal future with respect to the metric $g_{\lambda}$.
} 


\section{ESTIMATES FOR THE SCALAR FIELD}

6.1. Decomposition of the inverse metric. For the purpose of controlling the scalar field, we need to decompose $g^{-1}$. Our strategy is to write it as a sum of the following four terms

$$
g^{-1}=\left(g_{0}\right)^{-1}+g_{1}^{\prime}+g_{3}^{\prime}+\text { Error, }
$$

where $\left(g_{0}\right)^{-1}$ is the inverse of the background metric and for $a=1,3, g_{a}^{\prime}$ is defined as in (4.23). The "Error" term is then defined so that (6.1) holds.

In the following propositions, we will give precise estimates for $g_{1}^{\prime}, g_{3}^{\prime}$ and the "Error" term. Let us note that we will only need to control the inverse metric when it is multiplied with (the decomposed pieces of) the scalar field. Therefore, it suffices to obtain estimates on the compact set $B\left(0, R_{\text {supp }}\right)$ and we need not be concerned with the growth near infinity 25 .

Proposition 6.1. $g_{1}^{\prime}$ depends only on the background and can be decomposed as follows:

$$
\begin{aligned}
\left(g_{1}^{\prime}\right)^{\mu \nu}= & \sum_{\mathbf{A}} \lambda^{2}\left(G_{1,1, \mathbf{A}}\right)^{\mu \nu} \sin \left(\frac{u_{\mathbf{A}}}{\lambda}\right)+\sum_{\mathbf{A}} \lambda^{2}\left(G_{1,2, \mathbf{A}}\right)^{\mu \nu} \cos \left(\frac{2 u_{\mathbf{A}}}{\lambda}\right) \\
& +\sum_{ \pm} \sum_{\mathbf{A}} \sum_{\mathbf{B} \neq \mathbf{A}} \lambda^{2}\left(G_{1, b i l, \mathbf{A}, \mathbf{B}, \pm}\right)^{\mu \nu} \cos \left(\frac{u_{\mathbf{A}} \pm u_{\mathbf{B}}}{\lambda}\right),
\end{aligned}
$$

where each of $G_{1,1, \mathbf{A}}, G_{1,2, \mathbf{A}}$ and $G_{1, b i l, \mathbf{A}, \mathbf{B}, \pm}$ is compactly supported in $B\left(0, R_{\text {supp }}\right)$ and they satisfy the estimate

$$
\begin{aligned}
\left\|\left(G_{1,1, \mathbf{A}}, G_{1,2, \mathbf{A}}, G_{1, b i l, \mathbf{A}, \mathbf{B}, \pm}\right)\right\|_{\left(\left(H^{8} \cap C^{8}\right)\left(B\left(0, R_{\text {supp }}\right)\right)\right)^{3}} \\
\quad+\left\|\left(\partial_{t} G_{1,1, \mathbf{A}}, \partial_{t} G_{1,2, \mathbf{A}}, \partial_{t} G_{1, b i l, \mathbf{A}, \mathbf{B}, \pm}\right)\right\|_{\left(\left(H^{7} \cap C^{7}\right)\left(B\left(0, R_{\text {supp }}\right)\right)\right)^{3}} \leq C\left(C_{0}\right) \varepsilon^{2} .
\end{aligned}
$$

In particular, we have

$$
\sum_{k \leq 8} \lambda^{k}\left\|g_{1}^{\prime}\right\|_{\left(H^{k} \cap C^{k}\right)\left(B\left(0, R_{\text {supp }}\right)\right)}+\sum_{k \leq 7} \lambda^{k+1}\left\|\partial_{t} g_{1}^{\prime}\right\|_{\left(H^{k} \cap C^{k}\right)\left(B\left(0, R_{\text {supp }}\right)\right)} \leq C\left(C_{0}\right) \varepsilon^{2} \lambda^{2} .
$$

Proof. This is an immediate consequence of the definitions (4.23), (4.37) and the estimates on the background solution (see Corollary 3.5.).

Proposition 6.2. On the compact set $B\left(0, R_{\text {supp }}+1\right), g_{3}^{\prime}$ satisfies the following $H^{k}$ estimates

$$
\sum_{k \leq 3} \lambda^{k}\left\|g_{3}^{\prime}\right\|_{H^{2+k}\left(B\left(0, R_{\text {supp }}+1\right)\right)}+\sum_{k \leq 2} \lambda^{k+1}\left\|\partial_{t} g_{3}^{\prime}\right\|_{H^{1+k}\left(B\left(0, R_{\text {supp }}+1\right)\right)} \leq C\left(C_{1}\right) \varepsilon \lambda^{2},
$$

and the following $C^{k}$ estimates

$$
\sum_{k \leq 3} \lambda^{k}\left\|g_{3}^{\prime}\right\|_{C^{k}\left(B\left(0, R_{\text {supp }}+1\right)\right)}+\sum_{k \leq 2} \lambda^{k+1}\left\|\partial_{t} g_{3}^{\prime}\right\|_{C^{k}\left(B\left(0, R_{\text {supp }}+1\right)\right)} \leq C\left(C_{1}\right) \varepsilon \lambda^{2} .
$$

Proof. This is an immediate consequence of the definition of $g_{3}^{\prime}$ in (4.23) together with the bootstrap assumption (BA4) and the estimate (5.8).

Proposition 6.3. On the compact set $B\left(0, R_{\text {supp }}+1\right)$, the error term in (6.1) can be controlled in $H^{k}$ as follows:

$$
\begin{aligned}
\sum_{k \leq 5} \lambda^{k} \| & g^{-1}-\left(g_{0}\right)^{-1}-g_{1}^{\prime}-g_{3}^{\prime} \|_{H^{k}\left(B\left(0, R_{\text {supp }}+1\right)\right)} \\
& +\sum_{k \leq 4} \lambda^{k+1}\left\|\partial_{t}\left(g^{-1}-\left(g_{0}\right)^{-1}-g_{1}^{\prime}-g_{3}^{\prime}\right)\right\|_{H^{k}\left(B\left(0, R_{\text {supp }}+1\right)\right)} \leq C\left(C_{1}\right) \varepsilon \lambda^{3},
\end{aligned}
$$

and in $C^{k}$ as follows:

$$
\begin{aligned}
\sum_{k \leq 3} \lambda^{k} \| & g^{-1}-\left(g_{0}\right)^{-1}-g_{1}^{\prime}-g_{3}^{\prime} \|_{C^{k}\left(B\left(0, R_{s u p p}+1\right)\right)} \\
& +\sum_{k \leq 2} \lambda^{k+1}\left\|\partial\left(g^{-1}-\left(g_{0}\right)^{-1}-g_{1}^{\prime}-g_{3}^{\prime}\right)\right\|_{C^{k}\left(B\left(0, R_{\text {supp }}+1\right)\right)} \leq C\left(C_{1}\right) \varepsilon \lambda^{2} .
\end{aligned}
$$

\footnotetext{
25 which, of course, is important for the estimates of the metric itself!
} 
Proof. $g_{1}^{\prime}$ and $g_{3}^{\prime}$ are defined so that $g^{-1}-\left(g_{0}\right)^{-1}-g_{1}^{\prime}-g_{3}^{\prime}$ can be expanded into terms which are either linear in $\mathfrak{g}_{2}$ or at least quadratic in $\mathfrak{g}_{1}, \mathfrak{g}_{2}$ and $\mathfrak{g}_{3}$. It is then easy to check that all these terms obey the desired bounds thanks to (B2), (BA3), (BA4), (5.7) and (5.8).

As a consequence of the decomposition (6.1), we also obtain the following estimate for $\square_{g}-\square_{g_{0}}$ :

Proposition 6.4. Let $f$ be compactly supported on $B\left(0, R_{\text {supp }}\right)$. Then, for $k \leq 4$,

$$
\begin{aligned}
& \left\|\left(\square_{g}-\square_{g_{0}}\right) f-\left(\partial_{\alpha}\left(g_{1}^{\prime}\right)^{\alpha \beta}+\left(g_{0}^{-1}\right)^{\alpha \beta}\left(2 \partial_{\alpha} \gamma_{1}+\frac{\partial_{\alpha} N_{1}}{N}\right)+\partial_{t}\left(g_{3}^{\prime}\right)^{t \beta}+\left(g_{0}^{-1}\right)^{t \beta} \frac{\partial_{t} N_{3}}{N_{0}}\right)\left(\partial_{\beta} f\right)\right\|_{H^{k}} \\
\leq & C\left(C_{1}\right) \varepsilon\left(\lambda\|\partial f\|_{H^{k}}+\lambda^{2}\left\|\partial^{2} f\right\|_{H^{k}}+\lambda^{2-k}\|\partial f\|_{L^{\infty}}+\lambda^{2-k}\left\|\partial^{2} f\right\|_{L^{\infty}}\right) .
\end{aligned}
$$

Proof. The difference of the wave operators takes the form

$$
\begin{aligned}
& \left(\square_{g}-\square_{g_{0}}\right) f=\underbrace{\left(\left(g^{-1}\right)^{\alpha \beta}-\left(g_{0}^{-1}\right)^{\alpha \beta}\right) \partial_{\alpha \beta}^{2} f}_{=: \text {Error }_{1}}+\underbrace{\left(\partial_{\alpha}\left(\left(g^{-1}\right)^{\alpha \beta}-\left(g_{0}^{-1}\right)^{\alpha \beta}\right)\right) \partial_{\beta} f}_{=: I} \\
& +\underbrace{\frac{1}{2}\left(\left(g^{-1}\right)^{\alpha \beta} \partial_{\alpha} \log |\operatorname{det} g|-\left(g_{0}^{-1}\right)^{\alpha \beta} \partial_{\alpha} \log \left|\operatorname{det} g_{0}\right|\right) \partial_{\beta} f}_{=: I I} .
\end{aligned}
$$

First, notice that Error 1 is an acceptable error 26 by Propositions 6.1, 6.2, 6.3 and A.8,

$$
\begin{aligned}
& \left\|\left(\left(g^{-1}\right)^{\alpha \beta}-\left(g_{0}^{-1}\right)^{\alpha \beta}\right) \partial_{\alpha \beta}^{2} f\right\|_{H^{k}} \\
\lesssim & \left\|\left(g^{-1}\right)-g_{0}^{-1}\right\|_{L^{\infty}\left(B\left(0, R_{\text {supp }}+1\right)\right)}\left\|\partial^{2} f\right\|_{H^{k}}+\left\|\partial^{2} f\right\|_{L^{\infty}}\left\|\left(g^{-1}\right)-g_{0}^{-1}\right\|_{H^{k}\left(B\left(0, R_{\text {supp }}+1\right)\right)} \\
\leq & C\left(C_{1}\right) \varepsilon \lambda^{2}\left\|\partial^{2} f\right\|_{H^{k}}+C\left(C_{1}\right) \varepsilon \lambda^{2-k}\left\|\partial^{2} f\right\|_{L^{\infty}} .
\end{aligned}
$$

Next, we consider the term $I$. By Proposition 6.3, it suffices to consider the contributions from $g_{1}^{\prime}$ and $g_{3}^{\prime}$ as the remainder is an acceptable error. The contribution from $g_{1}^{\prime}$ is precisely $\left(\partial_{\alpha}\left(g_{1}^{\prime}\right)^{\alpha \beta}\right)\left(\partial_{\beta} f\right)$. For the contribution from $g_{3}^{\prime}$, we have $\left(\partial_{\alpha}\left(g_{3}^{\prime}\right)^{\alpha \beta}\right)\left(\partial_{\beta} f\right)$. Then notice that by Proposition 6.2, if $\alpha=1$ or $\alpha=2$, this term is an acceptable error. More precisely, we estimate using Proposition A.8

$$
\begin{aligned}
\left\|\nabla g_{3}^{\prime} \partial f\right\|_{H^{k}} & \lesssim\left\|\nabla g_{3}^{\prime}\right\|_{L^{\infty}\left(B\left(0, R_{\text {supp }}+1\right)\right.}\|\partial f\|_{H^{k}}+\|\partial f\|_{L^{\infty}}\left\|\nabla g_{3}^{\prime}\right\|_{H^{k}\left(B\left(0, R_{\text {supp }}+1\right)\right.} \\
& \leq C\left(C_{1}\right) \varepsilon \lambda\|\partial f\|_{H^{k}}+C\left(C_{1}\right) \varepsilon \lambda^{2-k}\|\partial f\|_{L^{\infty}},
\end{aligned}
$$

where we have used (BA4) and (5.8). We are thus left with $\left(\partial_{t}\left(g_{3}^{\prime}\right)^{t \beta}\right)\left(\partial_{\beta} f\right)$, which is one of the main terms.

Finally, we compute the term $I I$ using (3.6) and suppress all the terms which according to Propositions 6.16 .2 and 6.3 can be treated as acceptable errors:

$$
\begin{aligned}
& \frac{1}{2}\left(\left(g^{-1}\right)^{\alpha \beta} \partial_{\alpha} \log |\operatorname{det} g|-\left(g_{0}^{-1}\right)^{\alpha \beta} \partial_{\alpha} \log \left|\operatorname{det} g_{0}\right|\right) \\
= & \left(g^{-1}\right)^{\alpha \beta} \partial_{\alpha}(2 \gamma+\log N)-\left(g_{0}^{-1}\right)^{\alpha \beta} \partial_{\alpha}\left(2 \gamma_{0}+\log N_{0}\right) \\
= & \left(g_{0}^{-1}\right)^{\alpha \beta} \partial_{\alpha}\left(2 \gamma_{1}+2 \gamma_{3}+\log \left(1+\frac{N_{1}+N_{3}}{N}\right)\right)+\left(\left(g_{1}^{\prime}\right)^{\alpha \beta}+\left(g_{3}^{\prime}\right)^{\alpha \beta}\right) \partial_{\alpha}\left(2 \gamma_{0}+\log N_{0}\right)+\ldots \\
= & \left(g_{0}^{-1}\right)^{\alpha \beta} \partial_{\alpha}\left(2 \gamma_{1}+2 \gamma_{3}\right)+\left(g_{0}^{-1}\right)^{\alpha \beta} \frac{\partial_{\alpha}\left(N_{1}+N_{3}\right)}{N+N_{1}+N_{3}}+\ldots \\
= & \left(g_{0}^{-1}\right)^{\alpha \beta}\left(2 \partial_{\alpha} \gamma_{1}+\frac{\partial_{\alpha} N_{1}}{N}\right)+\left(g_{0}^{-1}\right)^{t \beta} \frac{\partial_{t} N_{3}}{N_{0}}+\ldots,
\end{aligned}
$$

where in the last line we have used the fact that (according to (BA4) ) we only need to treat the $\partial_{t}$ derivative of $\mathfrak{g}_{3}$ and moreover that $\partial_{t} \gamma_{3}$ satisfies better bounds according to (BA5). More precisely, we estimate using Proposition A.8

$$
\left\|\partial_{t} \gamma_{3} \partial f\right\|_{H^{k}} \lesssim\left\|\partial_{t} \gamma_{3}\right\|_{L^{\infty}}\|\partial f\|_{H^{k}}+\|\partial f\|_{L^{\infty}}\left\|\partial_{t} \gamma_{3}\right\|_{H^{k}} \leq C\left(C_{1}\right) \varepsilon \lambda\|\partial f\|_{H^{k}}+C\left(C_{1}\right) \varepsilon \lambda^{2-k}\|\partial f\|_{L^{\infty}}
$$

where for $\left\|\partial_{t} \gamma_{3}\right\|_{L^{\infty}}$, we have used (5.8); and for $\left\|\partial_{t} \gamma_{3}\right\|_{H^{k}}$, we have used (BA5) for $k=0$ and (BA4) for $k \geq 1$.

We also have another variant of Proposition 6.4, which requires more integrability for derivatives of $f$ :

\footnotetext{
26 in the sense that it can be bounded by the RHS of 6.2 . We will also use the same language below without further comment.
} 
Proposition 6.5. Let $f$ be compactly supported on $B\left(0, R_{\text {supp }}\right)$. Then, for $k \leq 4$,

$$
\begin{aligned}
& \left\|\left(\square_{g}-\square_{g_{0}}\right) f-\left(\partial_{\alpha}\left(g_{1}^{\prime}\right)^{\alpha \beta}+\left(g_{0}^{-1}\right)^{\alpha \beta}\left(2 \partial_{\alpha} \gamma_{1}+\frac{\partial_{\alpha} N_{1}}{N}\right)+\partial_{t}\left(g_{3}^{\prime}\right)^{t \beta}+\left(g_{0}^{-1}\right)^{t \beta} \frac{\partial_{t} N_{3}}{N_{0}}\right)\left(\partial_{\beta} f\right)\right\|_{H^{k}} \\
\leq & C\left(C_{1}\right) \varepsilon \sum_{\ell \leq k} \lambda^{2-\ell}\left(\left\|\partial^{2} f\right\|_{C^{k-\ell}}+\|\partial f\|_{C^{k-\ell}}\right) .
\end{aligned}
$$

Proof. Since $\operatorname{supp}(f) \subset B\left(0, R_{\text {supp }}\right),\|\partial f\|_{H^{k}} \lesssim\|\partial f\|_{C^{k}}$ and $\left\|\partial^{2} f\right\|_{H^{k}} \lesssim\left\|\partial^{2} f\right\|_{C^{k}}$. We can therefore proceed in the same way as in the proof of Proposition 6.4 except that we need to improve the estimate (6.3) and (6.4) in terms of the power of $\lambda$.

For (6.3), we have

$$
\left\|\nabla g_{3}^{\prime} \partial f\right\|_{H^{k}} \lesssim \sum_{\ell \leq k}\left\|\nabla g_{3}^{\prime}\right\|_{H^{\ell}}\|\partial f\|_{C^{k-\ell}} \leq C\left(C_{1}\right) \varepsilon \sum_{\ell \leq k} \lambda^{2-\ell}\|\partial f\|_{C^{k-\ell}},
$$

where we have used (BA4). For (6.4), we have

$$
\left\|\partial_{t} \gamma_{3} \partial f\right\|_{H^{k}} \lesssim \sum_{\ell \leq k}\left\|\partial_{t} \gamma_{3}\right\|_{H^{\ell}}\|\partial f\|_{C^{k-\ell}} \leq C\left(C_{1}\right) \varepsilon \sum_{\ell \leq k} \lambda^{2-\ell}\|\partial f\|_{C^{k-\ell}}
$$

where we used (BA4) for $\ell \geq 1$ and (BA5) for $\ell=0$.

6.2. Computations for the main terms (and definitions of $A_{\mathbf{A}}^{(1)}, A_{\mathbf{A}}^{(2)}, A_{\mathbf{A}}^{(3)}$ ). In this subsection, we compute the $\square_{g}$ of the main part of $\phi_{\lambda}$, i.e., of $\phi_{\lambda}-\mathcal{E}_{\lambda}$. We will show that it consists either of (1) terms of size $C\left(C_{1}\right) \varepsilon \lambda^{2}$ or (2) terms of size $C\left(C_{0}\right) \varepsilon^{2} \lambda$ but oscillating in a non-null direction or (3) terms of size $C\left(C_{1}\right) \varepsilon \lambda$ and roughly of the form $\partial_{t} \mathfrak{g}_{3}$ multiplied by a regular function of size $C\left(C_{0}\right) \varepsilon$. The main result of the section is given in Proposition 6.13

In the process of obtaining Proposition 6.13, we will define $A_{\mathbf{A}}^{(1)}, A_{\mathbf{A}}^{(2)}, A_{\mathbf{A}}^{(3)}$, which appeared in the definitions (4.19), (4.20) and (4.21) (see Definition 6.8). As we will see, they are defined so that certain cancellations take place.

Proposition 6.6. There exist functions $A_{\mathbf{A}}^{(1)}\left[\phi_{0}\right], A_{\mathbf{A}}^{(2)}\left[\phi_{0}\right]$ and $B_{\mathbf{A B}}^{(2, \pm)}\left[\phi_{0}\right]$, each of which depends only on the background solution, is compactly supported in $B\left(0, R_{\text {supp }}\right)$ and obeys the bound

$$
\|\cdot\|_{H^{8} \cap C^{8}}+\left\|\partial_{t}(\cdot)\right\|_{H^{7} \cap C^{7}}+\left\|\partial_{t}^{2}(\cdot)\right\|_{H^{6} \cap C^{6}} \leq C\left(C_{0}\right) \varepsilon^{3}
$$

such that

$$
\begin{aligned}
\square_{g} \phi_{0}= & \left(\partial_{t}\left(g_{3}^{\prime}\right)^{t \beta}+\left(g_{0}^{-1}\right)^{t \beta} \frac{\partial_{t} N_{3}}{N_{0}}\right)\left(\partial_{\beta} \phi_{0}\right)+\lambda \sum_{\mathbf{A}}\left(A_{\mathbf{A}}^{(1)}\left[\phi_{0}\right] \cos \left(\frac{u_{\mathbf{A}}}{\lambda}\right)+A_{\mathbf{A}}^{(2)}\left[\phi_{0}\right] \sin \left(\frac{2 u_{\mathbf{A}}}{\lambda}\right)\right) \\
& +\lambda \sum_{ \pm} \sum_{\mathbf{A}} \sum_{\mathbf{B} \neq \mathbf{A}} B_{\mathbf{A B}}^{(2, \pm)}\left[\phi_{0}\right] \sin \left(\frac{u_{\mathbf{A}} \pm u_{\mathbf{B}}}{\lambda}\right)+R\left[\phi_{0}\right],
\end{aligned}
$$

where $R\left[\phi_{0}\right]$ is compactly supported in $B\left(0, R_{\text {supp }}\right)$ and satisfies the following estimate:

$$
\sum_{k \leq 3} \lambda^{k}\left\|R\left[\phi_{0}\right]\right\|_{H^{k}} \leq C\left(C_{1}\right) \varepsilon \lambda^{2} .
$$

Proof. Since $\square_{g_{0}} \phi_{0}=0$, it suffices to compute $\left(\square_{g}-\square_{g_{0}}\right) \phi_{0}$. Using Corollary 3.5 and Proposition 6.5.

$$
\begin{aligned}
& \square_{g} \phi_{0}=\left(\square_{g}-\square_{g_{0}}\right) \phi_{0} \\
= & \left(\partial_{\alpha}\left(g_{1}^{\prime}\right)^{\alpha \beta}+\left(g_{0}^{-1}\right)^{\alpha \beta}\left(2 \partial_{\alpha} \gamma_{1}+\frac{\partial_{\alpha} N_{1}}{N}\right)+\partial_{t}\left(g_{3}^{\prime}\right)^{t \beta}+\left(g_{0}^{-1}\right)^{t \beta} \frac{\partial_{t} N_{3}}{N_{0}}\right)\left(\partial_{\beta} \phi_{0}\right)+\ldots,
\end{aligned}
$$

where ... denotes terms that in the $\sum_{k \leq 3} \lambda^{k}\|\cdot\|_{H^{k}}$ norm is bounded by $C\left(C_{1}\right) \varepsilon \lambda^{2}$. These terms are then treated as $R\left[\phi_{0}\right]$.

Notice that in (6.6), the terms involving $\partial_{t} g_{3}^{\prime}$ and $\partial_{t} N_{3}$ are exactly of the form in the statement of the proposition. It thus remains to consider the terms that involve the derivative of $g_{1}^{\prime}, \gamma_{1}$ and $N_{1}$. Now by (4.37), (B2) and Proposition 6.1, $g_{1}^{\prime}, \gamma_{1}$ and $N_{1}$ can be written as sum of terms with oscillating factors $\sin \left(\frac{u_{\mathbf{A}}}{\lambda}\right), \cos \left(\frac{2 u_{\mathbf{A}}}{\lambda}\right)$ and $\cos \left(\frac{u_{\mathbf{A}} \pm u_{\mathbf{B}}}{\lambda}\right)($ with $\mathbf{A} \neq \mathbf{B})$ with coefficients which in the $\|\cdot\|_{H^{8} \cap C^{8}}+\left\|\partial_{t}(\cdot)\right\|_{H^{7} \cap C^{7}}$ 
norm are of size $C\left(C_{0}\right) \varepsilon^{2} \lambda^{2}$. Therefore, their derivatives, when multiplied by the derivatives of $\phi_{0}$, takes the form

$$
\lambda \sum_{\mathbf{A}}\left(A_{\mathbf{A}}^{(1)}\left[\phi_{0}\right] \cos \left(\frac{u_{\mathbf{A}}}{\lambda}\right)+A_{\mathbf{A}}^{(2)}\left[\phi_{0}\right] \sin \left(\frac{2 u_{\mathbf{A}}}{\lambda}\right)\right)+\lambda \sum_{ \pm} \sum_{\mathbf{A}} \sum_{\mathbf{B} \neq \mathbf{A}} B_{\mathbf{A B}}^{(2, \pm)}\left[\phi_{0}\right] \sin \left(\frac{u_{\mathbf{A}} \pm u_{\mathbf{B}}}{\lambda}\right)+\ldots
$$

This concludes the proof of the proposition.

Proposition 6.7. There exist functions $A_{\mathbf{A}}^{(1)}[F], A_{\mathbf{A}}^{(2)}[F], A_{\mathbf{A}}^{(3)}[F], A_{\mathbf{A B}}^{(\text {cross })}[F], B_{\mathbf{A B}}^{(2, \pm)}[F], B_{\mathbf{A B}}^{(3, \pm)}[F]$ and $D_{\mathrm{ABC}}^{\left( \pm_{1}, \pm_{2}\right)}[F]$, each of which depends only on the background solution, is compactly supported in $B\left(0, R_{\text {supp }}\right)$ and obeys the bound

$$
\|\cdot\|_{H^{8} \cap C^{8}}+\left\|\partial_{t}(\cdot)\right\|_{H^{7} \cap C^{7}}+\left\|\partial_{t}^{2}(\cdot)\right\|_{H^{6} \cap C^{6}} \leq C\left(C_{0}\right) \varepsilon^{3}
$$

such that for every $\mathbf{A} \in \mathcal{A}$,

$$
\begin{aligned}
& \square_{g}\left(\lambda F_{\mathbf{A}} \cos \left(\frac{u_{\mathbf{A}}}{\lambda}\right)\right) \\
= & \left(\lambda \square_{g_{0}} F_{\mathbf{A}}-\frac{1}{\lambda}\left(g_{3}^{\prime}\right)^{\alpha \beta} \partial_{\alpha} u_{\mathbf{A}} \partial_{\beta} u_{\mathbf{A}} F_{\mathbf{A}}\right) \cos \left(\frac{u_{\mathbf{A}}}{\lambda}\right)-\left(\partial_{t}\left(g_{3}^{\prime}\right)^{t \beta}+\left(g_{0}^{-1}\right)^{t \beta} \frac{\partial_{t} N_{3}}{N_{0}}\right)\left(\partial_{\beta} u_{\mathbf{A}}\right) F_{\mathbf{A}} \sin \left(\frac{u_{\mathbf{A}}}{\lambda}\right) \\
& +\lambda A_{\mathbf{A}}^{(1)}[F] \cos \left(\frac{u_{\mathbf{A}}}{\lambda}\right)+\lambda A_{\mathbf{A}}^{(2)}[F] \sin \left(\frac{2 u_{\mathbf{A}}}{\lambda}\right)+\lambda A_{\mathbf{A}}^{(3)}[F] \cos \left(\frac{3 u_{\mathbf{A}}}{\lambda}\right)+\sum_{\mathbf{B} \neq \mathbf{A}} \lambda A_{\mathbf{A B}}^{(\text {cross })}[F] \cos \left(\frac{u_{\mathbf{B}}}{\lambda}\right) \\
& +\sum_{ \pm} \sum_{\mathbf{B} \neq \mathbf{A}} \lambda B_{\mathbf{A B}}^{(2, \pm)}[F] \sin \left(\frac{u_{\mathbf{A}} \pm u_{\mathbf{B}}}{\lambda}\right)+\sum_{ \pm} \sum_{\mathbf{B}} \lambda B_{\mathbf{A B}}^{(3, \pm)}[F] \cos \left(\frac{u_{\mathbf{A}} \pm 2 u_{\mathbf{B}}}{\lambda}\right) \\
& +\sum_{ \pm_{1}} \sum_{ \pm_{2}} \sum_{\mathbf{B} \neq \mathbf{A}} \sum_{\substack{\mathbf{C} \neq \mathbf{B} \\
\mathbf{C} \neq \mathbf{A}}} \lambda D_{\mathbf{A B C}}^{\left( \pm_{1}, \pm_{2}\right)}[F] \cos \left(\frac{u_{\mathbf{A}} \pm_{1} u_{\mathbf{B}} \pm_{2} u_{\mathbf{C}}}{\lambda}\right)+R_{\mathbf{A}}[F],
\end{aligned}
$$

where $R_{\mathbf{A}}[F]$ is compactly supported in $B\left(0, R_{\text {supp }}\right)$ and is small in the following sense:

$$
\sum_{k \leq 3} \lambda^{k}\left\|R_{\mathbf{A}}[F]\right\|_{H^{k}} \leq C\left(C_{1}\right) \varepsilon \lambda^{2} .
$$

Proof. Using the expansion (6.1), the estimate in Proposition 6.3 and the fact that $\left(g_{0}^{-1}\right)^{\alpha \beta} \partial_{\alpha} u_{\mathbf{A}} \partial_{\beta} u_{\mathbf{A}}=0$ and $\left(\square_{g_{0}} u_{\mathbf{A}}\right) F_{\mathbf{A}}+2\left(g_{0}^{-1}\right)^{\alpha \beta} \partial_{\alpha} u_{\mathbf{A}} \partial_{\beta} F_{\mathbf{A}}=0$ (recall that $u_{\mathbf{A}}$ and $F_{\mathbf{A}}$ satisfy (1.4)), we get

$$
\begin{aligned}
& \square_{g}\left(\lambda F_{\mathbf{A}} \cos \left(\frac{u_{\mathbf{A}}}{\lambda}\right)\right) \\
= & \lambda\left(\square_{g} F_{\mathbf{A}}\right) \cos \left(\frac{u_{\mathbf{A}}}{\lambda}\right)-2\left(g^{-1}\right)^{\alpha \beta} \partial_{\alpha} u_{\mathbf{A}} \partial_{\beta} F_{\mathbf{A}} \sin \left(\frac{u_{\mathbf{A}}}{\lambda}\right) \\
& -\left(\square_{g} u_{\mathbf{A}}\right) F_{\mathbf{A}} \sin \left(\frac{u_{\mathbf{A}}}{\lambda}\right)-\frac{1}{\lambda}\left(g^{-1}\right)^{\alpha \beta} \partial_{\alpha} u_{\mathbf{A}} \partial_{\beta} u_{\mathbf{A}} F_{\mathbf{A}} \cos \left(\frac{u_{\mathbf{A}}}{\lambda}\right) \\
= & \underbrace{\lambda\left(\square_{g_{0}} F_{\mathbf{A}}\right) \cos \left(\frac{u_{\mathbf{A}}}{\lambda}\right)}_{=: \widetilde{F}_{1}} \underbrace{-\frac{1}{\lambda}\left(g_{1}^{\prime}\right)^{\alpha \beta} \partial_{\alpha} u_{\mathbf{A}} \partial_{\beta} u_{\mathbf{A}} F_{\mathbf{A}} \cos \left(\frac{u_{\mathbf{A}}}{\lambda}\right)}_{=: \widetilde{F}_{4}} \underbrace{-\frac{1}{\lambda}\left(g_{3}^{\prime}\right)^{\alpha \beta} \partial_{\alpha} u_{\mathbf{A}} \partial_{\beta} u_{\mathbf{A}} F_{\mathbf{A}} \cos \left(\frac{u_{\mathbf{A}}}{\lambda}\right)}_{=: \widetilde{F}_{2}} \\
& \underbrace{-\left(\left(\square_{g}-\square_{g_{0}}\right) u_{\mathbf{A}}\right) F_{\mathbf{A}}}_{=: \widetilde{\mathfrak{F}}_{3}} \sin \left(\frac{u_{\mathbf{A}}}{\lambda}\right)+\ldots,
\end{aligned}
$$

where we have used the convention (as in the proof of Proposition 6.6) that ... denotes terms which are compactly supported in $B\left(0, R_{\text {supp }}\right)$ that by Propositions 6.3 and 6.4 are bounded in the $\sum_{k \leq 3} \lambda^{k}\|\cdot\|_{H^{k}}$ norm by $C\left(C_{1}\right) \varepsilon \lambda^{2}$. These terms can then be grouped into $R_{\mathbf{A}}[F]$.

This computation already shows that the potentially most harmful term of order $O\left(\frac{\varepsilon}{\lambda}\right)$ is not present since $u_{\mathbf{A}}$ is an eikonal function of the background solution. Also, an $O(\varepsilon)$ term is not present thanks to $\left(\square_{g_{0}} u_{\mathbf{A}}\right) F_{\mathbf{A}}+2\left(g_{0}^{-1}\right)^{\alpha \beta} \partial_{\alpha} u_{\mathbf{A}} \partial_{\beta} F_{\mathbf{A}}=0$. The remaining terms are of size at most $O(\varepsilon \lambda)$. This smallness will however not be sufficient to close the argument and we will need to analyze all the $O(\varepsilon \lambda)$ terms, only allowing $O\left(\varepsilon \lambda^{2}\right)$ terms to be treated as error terms.

The terms $\mathfrak{F}_{1}$ and $\mathfrak{F}_{3}$. First, there are the two main terms $\mathfrak{F}_{1}$ and $\mathfrak{F}_{3}$, which are simply included into the main terms in the statement of the proposition. 
The term $\mathfrak{F}_{2}$. Using Proposition 6.1, we expand $\mathfrak{F}_{2}$ as follow 27:

$$
\begin{aligned}
\mathfrak{F}_{2}= & -\lambda \sum_{\mathbf{B}}\left(G_{1,1, \mathbf{B}}\right)^{\alpha \beta} \partial_{\alpha} u_{\mathbf{A}} \partial_{\beta} u_{\mathbf{A}} F_{\mathbf{A}} \cos \left(\frac{u_{\mathbf{A}}}{\lambda}\right) \sin \left(\frac{u_{\mathbf{B}}}{\lambda}\right) \\
& -\lambda \sum_{\mathbf{B}}\left(G_{1,2, \mathbf{B}}\right)^{\alpha \beta} \partial_{\alpha} u_{\mathbf{A}} \partial_{\beta} u_{\mathbf{A}} F_{\mathbf{A}} \cos \left(\frac{u_{\mathbf{A}}}{\lambda}\right) \cos \left(\frac{2 u_{\mathbf{B}}}{\lambda}\right) \\
& -\sum_{ \pm} \sum_{\mathbf{B}} \sum_{\mathbf{C} \neq \mathbf{B}} \lambda\left(G_{1, b i l, \mathbf{B}, \mathbf{C}, \pm}\right)^{\alpha \beta} \partial_{\alpha} u_{\mathbf{A}} \partial_{\beta} u_{\mathbf{A}} F_{\mathbf{A}} \cos \left(\frac{u_{\mathbf{A}}}{\lambda}\right) \cos \left(\frac{u_{\mathbf{B}} \pm u_{\mathbf{C}}}{\lambda}\right) .
\end{aligned}
$$

We can expand this using standard trigonometric identities. First, notice that there are no low frequency terms. We then separate terms which according to whether $\mathbf{B} \neq \mathbf{A}$ (and $\mathbf{B} \neq \mathbf{C}$ ). It is easy to check that each of the terms takes the form of one of the terms in the statement of the proposition.

The term $\mathfrak{F}_{4}$. Finally, for the term $\mathfrak{F}_{4}$, we apply Proposition 6.5 to get

$$
\mathfrak{F}_{4}=-\left(\partial_{\alpha}\left(g_{1}^{\prime}\right)^{\alpha \beta}+\left(g_{0}^{-1}\right)^{\alpha \beta}\left(2 \partial_{\alpha} \gamma_{1}+\frac{\partial_{\alpha} N_{1}}{N}\right)+\partial_{t}\left(g_{3}^{\prime}\right)^{t \beta}+\left(g_{0}^{-1}\right)^{t \beta} \frac{\partial_{t} N_{3}}{N_{0}}\right)\left(\partial_{\beta} u_{\mathbf{A}}\right) F_{\mathbf{A}} \sin \left(\frac{u_{\mathbf{A}}}{\lambda}\right)+\ldots
$$

The term $-\left(\partial_{t}\left(g_{3}^{\prime}\right)^{t \beta}+\left(g_{0}^{-1}\right)^{t \beta} \frac{\partial_{t} N_{3}}{N_{0}}\right)\left(\partial_{\beta} u_{\mathbf{A}}\right) F_{\mathbf{A}} \sin \left(\frac{u_{\mathbf{A}}}{\lambda}\right)$ is one of the main terms in the statement of the proposition. The remaining terms involving the derivatives of $g_{1}^{\prime}, \gamma_{1}$ and $N_{1}$ can, according to (4.37) and Proposition 6.1, be written as a sum of terms with oscillating factors $\sin \left(\frac{u_{\mathrm{A}}}{\lambda}\right) \cos \left(\frac{u_{\mathrm{B}}}{\lambda}\right), \sin \left(\frac{u_{\mathrm{A}}}{\lambda}\right) \sin \left(\frac{2 u_{\mathrm{B}}}{\lambda}\right)$ and $\sin \left(\frac{u_{\mathrm{A}}}{\lambda}\right) \sin \left(\frac{u_{\mathbf{B}} \pm u_{\mathbf{C}}}{\lambda}\right)($ with $\mathbf{B} \neq \mathbf{C})$ with coefficients which in the $\|\cdot\|_{H^{8} \cap C^{8}}+\left\|\partial_{t}(\cdot)\right\|_{H^{7} \cap C^{7}}$ norm are of size $C\left(C_{0}\right) \varepsilon^{3} \lambda$. Expanding again with standard trigonometric identitie 28 , it is easy to check that each of the terms takes the form of one of the terms in the statement of the proposition.

This concludes the proof of the proposition.

We can now define $A_{\mathbf{A}}^{(1)}, A_{\mathbf{A}}^{(2)}$ and $A_{\mathbf{A}}^{(3)}$, which appeared in the definitions (4.19), (4.20) and (4.21):

Definition 6.8. Let $A_{\mathbf{A}}^{(1)}, A_{\mathbf{A}}^{(2)}$ and $A_{\mathbf{A}}^{(3)}$ be defined $a \sqrt{29}$

$$
\begin{aligned}
& A_{\mathbf{A}}^{(1)}:=-\left(A_{\mathbf{A}}^{(1)}\left[\phi_{0}\right]+A_{\mathbf{A}}^{(1)}[F]+\sum_{\mathbf{B} \neq \mathbf{A}} A_{\mathbf{B A}}^{(\text {cross })}[F]\right), \\
& A_{\mathbf{A}}^{(2)}:=\frac{1}{2}\left(A_{\mathbf{A}}^{(2)}\left[\phi_{0}\right]+A_{\mathbf{A}}^{(2)}[F]\right), \\
& A_{\mathbf{A}}^{(3)}:=-\frac{1}{3} A_{\mathbf{A}}^{(3)}[F],
\end{aligned}
$$

where all the terms on the RHS are as in Propositions 6.6 and 6.7

It is obvious that $A_{\mathbf{A}}^{(1)}, A_{\mathbf{A}}^{(2)}$ and $A_{\mathbf{A}}^{(3)}$ obey the bounds as asserted in (4.22). We collect them in the following proposition:

Proposition 6.9. $A_{\mathbf{A}}^{(1)}, A_{\mathbf{A}}^{(2)}$ and $A_{\mathbf{A}}^{(3)}$ obey the bound

$$
\left\|A_{\mathbf{A}}^{(a)}\right\|_{H^{8} \cap C^{8}}+\left\|\partial_{t} A_{\mathbf{A}}^{(a)}\right\|_{H^{7} \cap C^{7}} \leq C\left(C_{0}\right) \varepsilon^{3} \quad \text { for } a=1,2,3 .
$$

Proof. This is immediate from Propositions 6.6 and 6.7

Proposition 6.10. For every $\mathbf{A} \in \mathcal{A}, \square_{g}\left(\lambda^{2} \widetilde{F}_{\mathbf{A}} \sin \left(\frac{u_{\mathbf{A}}}{\lambda}\right)\right)$ can be written as follows:

$$
\begin{aligned}
& \square_{g}\left(\lambda^{2} \widetilde{F}_{\mathbf{A}} \sin \left(\frac{u_{\mathbf{A}}}{\lambda}\right)\right)-\left(\frac{1}{\lambda} F_{\mathbf{A}}\left(g_{3}^{\prime}\right)^{\alpha \beta} \partial_{\alpha} u_{\mathbf{A}} \partial_{\beta} u_{\mathbf{A}}-\lambda \square_{g_{0}} F_{\mathbf{A}}+\lambda A_{\mathbf{A}}^{(1)}\right) \cos \left(\frac{u_{\mathbf{A}}}{\lambda}\right) \\
= & \lambda^{2}\left(g_{0}^{-1}\right)^{t t} \partial_{t}^{2} \widetilde{F}_{\mathbf{A}} \sin \left(\frac{u_{\mathbf{A}}}{\lambda}\right)+R_{\mathbf{A}}[\widetilde{F}],
\end{aligned}
$$

where $R_{\mathbf{A}}[\widetilde{F}]$ denotes terms that are compactly supported in $B\left(0, R_{\text {supp }}\right)$ and satisfy the following estimate:

$$
\sum_{k \leq 3} \lambda^{k}\left\|R_{\mathbf{A}}[\widetilde{F}]\right\|_{H^{k}} \leq C\left(C_{1}\right) \varepsilon \lambda^{2} .
$$

\footnotetext{
${ }^{27}$ Note that we have relabeled the indices in Proposition 6.1

${ }^{28}$ Let us note that compared to the terms in $\mathfrak{F}_{2}$, the roles of all the sin and cos are flipped and therefore the products of these trigonometric functions still take the same form.

${ }^{29}$ Note that the swapping of the indices in $A^{(\text {cross })}$ as compared to Proposition 6.7 is intentional.
} 
Proof. Using $\left(g_{0}^{-1}\right)^{\alpha \beta} \partial_{\alpha} u_{\mathbf{A}} \partial_{\beta} u_{\mathbf{A}}=0$ and (4.19), we have

$$
\begin{aligned}
& \square_{g}\left(\lambda^{2} \widetilde{F}_{\mathbf{A}} \sin \left(\frac{u_{\mathbf{A}}}{\lambda}\right)\right) \\
= & \lambda^{2}\left(\square_{g_{0}} \widetilde{F}_{\mathbf{A}}\right) \sin \left(\frac{u_{\mathbf{A}}}{\lambda}\right)+\lambda\left(2\left(g_{0}^{-1}\right)^{\alpha \beta} \partial_{\alpha} u_{\mathbf{A}} \partial_{\beta} \widetilde{F}_{\mathbf{A}}+\left(\square_{g_{0}} u_{\mathbf{A}}\right) \widetilde{F}_{\mathbf{A}}\right) \cos \left(\frac{u_{\mathbf{A}}}{\lambda}\right) \\
& +\left(\square_{g}-\square_{g_{0}}\right)\left(\lambda^{2} \widetilde{F}_{\mathbf{A}} \sin \left(\frac{u_{\mathbf{A}}}{\lambda}\right)\right) \\
= & \underbrace{\lambda^{2}\left(\square_{g_{0}} \widetilde{F}_{\mathbf{A}}\right) \sin \left(\frac{u_{\mathbf{A}}}{\lambda}\right)}_{=: \widetilde{\mathfrak{F}}_{1}}+\underbrace{\left(\frac{1}{\lambda} F_{\mathbf{A}}\left(g_{3}^{\prime}\right)^{\alpha \beta} \partial_{\alpha} u_{\mathbf{A}} \partial_{\beta} u_{\mathbf{A}}-\lambda \square_{g_{0}} F_{\mathbf{A}}+\lambda A_{\mathbf{A}}^{(1)}\right) \cos \left(\frac{u_{\mathbf{A}}}{\lambda}\right)}_{=: \widetilde{\mathfrak{F}}_{2}} \\
& +\underbrace{\left(\square_{g}-\square_{g_{0}}\right)\left(\lambda^{2} \widetilde{F}_{\mathbf{A}} \sin \left(\frac{u_{\mathbf{A}}}{\lambda}\right)\right)}_{=: \widetilde{\mathfrak{F}}_{3}} .
\end{aligned}
$$

For the term $\widetilde{\mathfrak{F}}_{1}$, notice that according to the bootstrap assumption (BA1), this is an acceptable error unless both derivatives on $\widetilde{F}_{\mathbf{A}}$ are $\partial_{t}$ derivatives, i.e.,

$$
\widetilde{\mathfrak{F}}_{1}=\lambda^{2}\left(g_{0}^{-1}\right)^{t t} \partial_{t}^{2} \widetilde{F}_{\mathbf{A}} \sin \left(\frac{u_{\mathbf{A}}}{\lambda}\right)+\ldots,
$$

which gives the main term on the RHS in the statement of the proposition.

The term $\widetilde{\mathfrak{F}}_{2}$ contributes to the main terms on the LHS in the statement of the proposition.

Finally, we claim that according to Proposition 6.4, the bootstrap assumptions and Proposition [5.3. $\widetilde{\mathfrak{F}}_{3}$ can be considered as part of $R_{\mathbf{A}}[\widetilde{F}]$. To see this, we first estimate the term on the RHS of (6.2) in Proposition 6.4 (with $f=\lambda^{2} \widetilde{F}_{\mathbf{A}} \sin \left(\frac{u_{\mathbf{A}}}{\lambda}\right)$ ),

$$
\begin{aligned}
& C\left(C_{1}\right) \varepsilon\left(\lambda\|\partial f\|_{H^{k}}+\lambda^{2}\left\|\partial^{2} f\right\|_{H^{k}}+\lambda^{2-k}\|\partial f\|_{L^{\infty}}+\lambda^{2-k}\left\|\partial^{2} f\right\|_{L^{\infty}}\right) \\
\leq & C\left(C_{1}\right) \varepsilon \sum_{k_{1}+k_{2}=k+1} \lambda^{3-k_{1}}\left\|\widetilde{F}_{\mathbf{A}}\right\|_{H^{k_{2}}}+\sum_{k_{1}+k_{2}=k}\left(\lambda^{3-k_{1}}\left\|\partial_{t} \widetilde{F}_{\mathbf{A}}\right\|_{H^{k_{2}}}+\lambda^{4-k_{1}}\left\|\partial_{t}^{2} \widetilde{F}_{\mathbf{A}}\right\|_{H^{k_{2}}}\right) \\
& +\lambda^{2-k}\left(\left\|\widetilde{F}_{\mathbf{A}}\right\|_{L^{\infty}}+\lambda\left\|\partial \widetilde{F}_{\mathbf{A}}\right\|_{L^{\infty}}+\lambda^{2}\left\|\partial^{2} \widetilde{F}_{\mathbf{A}}\right\|_{L^{\infty}}\right) \leq C\left(C_{1}\right) \varepsilon^{2} \lambda^{2-k},
\end{aligned}
$$

where we have used (BA1), (5.4) and the bounds of $u_{\mathbf{A}}$ in Corollary 3.5. We then estimate the terms on the LHS in Proposition 6.4 (with $f=\lambda^{2} \widetilde{F}_{\mathbf{A}} \sin \left(\frac{u_{\mathbf{A}}}{\lambda}\right)$ ). By Corollary 3.5, (B2) and (BA1), we have

$$
\left\|\partial g_{1}^{\prime} \partial f\right\|_{H^{k}} \leq C\left(C_{0}\right) \varepsilon^{2} \sum_{k_{1}+k_{2}=k} \lambda^{1-k_{1}}\|\partial f\|_{H^{k_{2}}} \leq C\left(C_{1}\right) \varepsilon^{4} \sum_{k_{1}+k_{2}=k} \lambda^{2-k_{1}-k_{2}} \leq C\left(C_{1}\right) \varepsilon^{4} \lambda^{2-k},
$$

and by (BA1), (BA4), (5.4), (5.8) and Proposition A.8.

$$
\begin{aligned}
\left\|\partial_{t} g_{3}^{\prime} \partial f\right\|_{H^{k}} & \leq C\left(\left\|\partial_{t} g_{3}^{\prime}\right\|_{L^{\infty}\left(B\left(0, R_{\text {supp }}+1\right)\right.}\|\partial f\|_{H^{k}}+\left\|\partial_{t} g_{3}^{\prime}\right\|_{H^{k}\left(B\left(0, R_{s u p p}+1\right)\right.}\|\partial f\|_{L^{\infty}}\right) \\
& \leq C\left(C_{1}\right)\left(\varepsilon \lambda\|\partial f\|_{H^{k}}+\varepsilon \lambda^{1-k}\|\partial f\|_{L^{\infty}}\right) \leq C\left(C_{1}\right) \varepsilon^{2} \lambda^{2-k}
\end{aligned}
$$

Proposition 6.11. For every $\mathbf{A} \in \mathcal{A}, \square_{g}\left(\lambda^{2} \widetilde{F}_{\mathbf{A}}^{(2)} \cos \left(\frac{2 u_{\mathbf{A}}}{\lambda}\right)\right)$ and $\square_{g}\left(\lambda^{2} \widetilde{F}_{\mathbf{A}}^{(3)} \sin \left(\frac{3 u_{\mathbf{A}}}{\lambda}\right)\right)$ can be written as follows:

$$
\square_{g}\left(\lambda^{2} \widetilde{F}_{\mathbf{A}}^{(2)} \cos \left(\frac{2 u_{\mathbf{A}}}{\lambda}\right)\right)+2 \lambda A_{\mathbf{A}}^{(2)} \sin \left(\frac{2 u_{\mathbf{A}}}{\lambda}\right)=R_{\mathbf{A}}\left[\widetilde{F}^{(2)}\right]
$$

and

$$
\square_{g}\left(\lambda^{2} \widetilde{F}_{\mathbf{A}}^{(3)} \sin \left(\frac{3 u_{\mathbf{A}}}{\lambda}\right)\right)-3 \lambda A_{\mathbf{A}}^{(3)} \cos \left(\frac{3 u_{\mathbf{A}}}{\lambda}\right)=R_{\mathbf{A}}\left[\widetilde{F}^{(3)}\right],
$$

where for $a=2,3, R_{\mathbf{A}}\left[\widetilde{F}^{(a)}\right]$ denotes terms that are compactly supported in $B\left(0, R_{\text {supp }}\right)$ and satisfy the following estimate:

$$
\sum_{k \leq 3} \lambda^{k}\left\|R_{\mathbf{A}}\left[\widetilde{F}^{(a)}\right]\right\|_{H^{k}} \leq C\left(C_{1}\right) \varepsilon \lambda^{2}
$$


Proof. Using $\left(g_{0}^{-1}\right)^{\alpha \beta} \partial_{\alpha} u_{\mathbf{A}} \partial_{\beta} u_{\mathbf{A}}=0$ and (4.20), we have

$$
\begin{aligned}
& \square_{g}\left(\lambda^{2} \widetilde{F}_{\mathbf{A}}^{(2)} \cos \left(\frac{2 u_{\mathbf{A}}}{\lambda}\right)\right) \\
= & \lambda^{2} \square_{g_{0}} \widetilde{F}_{\mathbf{A}}^{(2)} \cos \left(\frac{2 u_{\mathbf{A}}}{\lambda}\right)-4 \lambda\left(g_{0}^{-1}\right)^{\alpha \beta} \partial_{\alpha} u_{\mathbf{A}} \partial_{\beta} \widetilde{F}_{\mathbf{A}}^{(2)} \sin \left(\frac{2 u_{\mathbf{A}}}{\lambda}\right) \\
& -2 \lambda \square_{g_{0}} u_{\mathbf{A}} \widetilde{F}_{\mathbf{A}}^{(3)} \sin \left(\frac{2 u_{\mathbf{A}}}{\lambda}\right)+\left(\square_{g}-\square_{g_{0}}\right)\left(\lambda^{2} \widetilde{F}_{\mathbf{A}}^{(2)} \cos \left(2 \frac{u_{\mathbf{A}}}{\lambda}\right)\right) \\
= & -2 \lambda A_{\mathbf{A}}^{(2)} \sin \left(\frac{2 u_{\mathbf{A}}}{\lambda}\right)+\ldots,
\end{aligned}
$$

where in the last line we have used (B1) and Proposition 6.4. In a similar manner, we have

$$
\square_{g}\left(\lambda^{2} \widetilde{F}_{\mathbf{A}}^{(3)} \sin \left(\frac{3 u_{\mathbf{A}}}{\lambda}\right)\right)=3 \lambda A_{\mathbf{A}}^{(3)} \cos \left(\frac{3 u_{\mathbf{A}}}{\lambda}\right)+\ldots
$$

Using Propositions 6.6 6.7, 6.10 and 6.11, we see that by the choice of $\widetilde{F}_{\mathbf{A}}, \widetilde{F}_{\mathbf{A}}^{(2)}$ and $\widetilde{F}_{\mathbf{A}}^{(3)}$, many of the terms cancel. However, Propositions 6.7 and 6.10 each has a term which we cannot control. Moreover, we cannot introduce additional terms in the parametrix to cancel these terms as we otherwise would not have sufficient regularity. Fortunately, there is the following additional cancellation!

Proposition 6.12. For every $\mathbf{A} \in \mathcal{A}$, the following estimate holds:

$$
\sum_{k \leq 3} \lambda^{k}\left\|\lambda^{2}\left(g_{0}^{-1}\right)^{t t} \partial_{t}^{2} \widetilde{F}_{\mathbf{A}}-\left(\partial_{t}\left(g_{3}^{\prime}\right)^{t \beta}+\left(g_{0}^{-1}\right)^{t \beta} \frac{\partial_{t} N_{3}}{N_{0}}\right) \partial_{\beta} u_{\mathbf{A}} F_{\mathbf{A}}\right\|_{H^{k}} \leq C\left(C_{1}\right) \varepsilon \lambda^{2} .
$$

Proof. By (3.7),

$$
\left(g_{0}^{-1}\right)^{t t}=-\frac{1}{N_{0}^{2}}, \quad\left(g_{0}^{-1}\right)^{t i}=\frac{\left(\beta_{0}\right)^{i}}{N_{0}^{2}} .
$$

By (4.23), we have

$$
\left(g_{3}^{\prime}\right)^{t t}=\frac{2 N_{3}}{N_{0}^{3}}, \quad\left(g_{3}^{\prime}\right)^{t i}=-\frac{2 N_{3} \beta_{0}^{i}}{N_{0}^{3}}+\frac{\beta_{3}^{i}}{N_{0}^{2}}, \quad\left(g_{3}^{\prime}\right)^{i j}=\frac{2 N_{3}}{N_{0}^{3}} \beta_{0}^{i} \beta_{0}^{j}-\frac{1}{N_{0}^{2}}\left(\beta_{0}^{i} \beta_{3}^{j}+\beta_{3}^{i} \beta_{0}^{j}\right)+e^{-2 \gamma_{0}}\left(-1+e^{-2 \gamma}\right) .
$$

Therefore, up to terms which are acceptable error terms, we have

$$
\begin{aligned}
& \left(\partial_{t}\left(g_{3}^{\prime}\right)^{t \beta}+\left(g_{0}^{-1}\right)^{t \beta} \frac{\partial_{t} N_{3}}{N_{0}}\right) \partial_{\beta} u_{\mathbf{A}} F_{\mathbf{A}} \\
= & \left(\frac{\partial_{t} N_{3}}{N_{0}^{3}} \partial_{t} u_{\mathbf{A}}-\frac{\left(\partial_{t} N_{3}\right) \beta_{0}^{i}}{N_{0}^{3}} \partial_{i} u_{\mathbf{A}}+\frac{\partial_{t} \beta_{3}^{i}}{N_{0}^{2}} \partial_{i} u_{\mathbf{A}}\right) F_{\mathbf{A}}+\ldots
\end{aligned}
$$

Notice that we have used in the above computation the observation that whenever $\partial_{t}$ acts on a background $\mathfrak{g}_{0}$, the term is an acceptable error. This observation will also be used later without further comments.

To compute $\partial_{t}^{2} \widetilde{F}_{\mathbf{A}}$, we begin with (4.19). Differentiating (4.19) by $\partial_{t}$, we notice that by the estimates on the background solution and the bounds on $A_{\mathbf{A}}^{(1)}$, the only terms which are not acceptable error are those where $\partial_{t}$ acts on $\widetilde{F}_{\mathbf{A}}$ or $g_{3}^{\prime}$. Therefore, suppressing acceptable error terms, we have

$$
2\left(g_{0}^{-1}\right)^{\alpha \beta} \partial_{\alpha} u_{\mathbf{A}} \partial_{\beta} \partial_{t} \widetilde{F}_{\mathbf{A}}=\frac{1}{\lambda^{2}} F_{\mathbf{A}} \partial_{t}\left(g_{3}^{\prime}\right)^{\alpha \beta} \partial_{\alpha} u_{\mathbf{A}} \partial_{\beta} u_{\mathbf{A}}+\ldots
$$

Notice now that the LHS of (6.10) consists of the term where $\beta=t$ and the other terms are acceptable error terms. On the other hand, for the RHS of (6.10), we compute using (6.8). Notice that the only terms that are not acceptable error terms are when $\partial_{t}$ acts on $\beta_{3}^{i}$ or $N_{3}$ - this is because by (BA5) (and (BA4)), $\partial_{t} \gamma_{3}$ obeys sufficiently good estimates. Therefore,

$$
\begin{aligned}
& -\frac{2}{N_{0}^{2}}\left(\partial_{t} u_{\mathbf{A}}-\beta_{0}^{i} \partial_{i} u_{\mathbf{A}}\right) \partial_{t}^{2} \widetilde{F}_{\mathbf{A}} \\
= & \frac{1}{\lambda^{2}} F_{\mathbf{A}}\left(\frac{2 \partial_{t} N_{3}}{N_{0}^{3}}\left(\partial_{t} u_{\mathbf{A}}-\beta_{0}^{i} \partial_{i} u_{\mathbf{A}}\right)^{2}+\frac{2\left(\partial_{t} \beta_{3}^{i}\right)}{N_{0}^{2}}\left(\partial_{t} u_{\mathbf{A}}-\beta_{0}^{j} \partial_{i} u_{\mathbf{A}}\right)\left(\partial_{i} u_{\mathbf{A}}\right)\right)+\ldots
\end{aligned}
$$


Since $\left|\nabla u_{\mathbf{A}}\right|>\frac{1}{4}$ and $u_{\mathbf{A}}$ is null with respect to $g_{0},\left(\partial_{t} u_{\mathbf{A}}-\beta_{0}^{i} \partial_{i} u_{\mathbf{A}}\right)$ is bounded away from 0 on the compact set $B\left(0, R_{\text {supp }}\right)$, on which $F_{\mathbf{A}}$ is supported. Therefore, after dividing (6.11) by $2 \lambda^{-2}\left(\partial_{t} u_{\mathbf{A}}-\beta_{0}^{i} \partial_{i} u_{\mathbf{A}}\right)$, we obtain

$$
\begin{aligned}
& \lambda^{2}\left(g_{0}^{-1}\right)^{t t} \partial_{t}^{2} \widetilde{F}_{\mathbf{A}}=-\frac{\lambda^{2}}{N_{0}^{2}} \partial_{t}^{2} \widetilde{F}_{\mathbf{A}} \\
= & F_{\mathbf{A}}\left(\frac{\partial_{t} N_{3}}{N_{0}^{3}}\left(\partial_{t} u_{\mathbf{A}}-\beta_{0}^{i} \partial_{i} u_{\mathbf{A}}\right)+\frac{\partial_{t} \beta_{3}^{i}}{N_{0}^{2}} \partial_{i} u_{\mathbf{A}}\right)+\ldots
\end{aligned}
$$

Finally, notice that there is an exact cancellation in the main terms in (6.9) and (6.12), which yields the proposition.

We now summarize all the calculations and arrive at the following main result of the subsection:

Proposition 6.13. There exist functions $B_{\mathrm{AB}}^{(2, \pm)}, B_{\mathrm{AB}}^{(3, \pm)}$ and $D_{\mathrm{ABC}}^{\left( \pm_{1}, \pm_{2}\right)}$, each of which depends only on the background solution, is compactly supported in $B\left(0, R_{\text {supp }}\right)$ and obeys the bound

$$
\|\cdot\|_{H^{8} \cap C^{8}}+\left\|\partial_{t}(\cdot)\right\|_{H^{7} \cap C^{7}}+\left\|\partial_{t}^{2}(\cdot)\right\|_{H^{6} \cap C^{6}} \leq C\left(C_{0}\right) \varepsilon^{3}
$$

such that

$$
\begin{aligned}
\square_{g}\left(\phi_{\lambda}-\mathcal{E}_{\lambda}\right)= & \left(\partial_{t}\left(g_{3}^{\prime}\right)^{t \beta}+\left(g_{0}^{-1}\right)^{t \beta} \frac{\partial_{t} N_{3}}{N_{0}}\right)\left(\partial_{\beta} \phi_{0}\right) \\
& +\sum_{ \pm} \sum_{\mathbf{A}} \sum_{\mathbf{B} \neq \mathbf{A}} \lambda B_{\mathbf{A B}}^{(2, \pm)} \sin \left(\frac{u_{\mathbf{A}} \pm u_{\mathbf{B}}}{\lambda}\right)+\sum_{ \pm} \sum_{\mathbf{A}} \sum_{\mathbf{B}} \lambda B_{\mathbf{A B}}^{(3, \pm)} \cos \left(\frac{u_{\mathbf{A}} \pm 2 u_{\mathbf{B}}}{\lambda}\right) \\
& +\sum_{ \pm_{1}} \sum_{ \pm_{2}} \sum_{\mathbf{A}} \sum_{\mathbf{B} \neq \mathbf{A}} \sum_{\substack{\mathbf{C} \neq \mathbf{B} \\
\mathbf{C} \neq \mathbf{A}}} \lambda D_{\mathbf{A B C}}^{\left( \pm_{1}, \pm_{2}\right)} \cos \left(\frac{u_{\mathbf{A}} \pm_{1} u_{\mathbf{B}} \pm_{2} u_{\mathbf{C}}}{\lambda}\right)+R,
\end{aligned}
$$

where $R$ is compactly supported in $B\left(0, R_{\text {supp }}\right)$ and satisfies the estimate

$$
\sum_{k \leq 3} \lambda^{k}\|R\|_{H^{k}} \leq C\left(C_{1}\right) \varepsilon \lambda^{2} .
$$

Proof. This is achieved by combining Propositions 6.6, 6.7, 6.10, 6.11 and 6.12, where we have defined

$$
B_{\mathrm{AB}}^{(2, \pm)}:=B_{\mathbf{A B}}^{(2, \pm)}\left[\phi_{0}\right]+B_{\mathbf{A B}}^{(2, \pm)}[F], \quad B_{\mathbf{A B}}^{(3, \pm)}:=B_{\mathbf{A B}}^{(3, \pm)}[F], \quad D_{\mathbf{A B C}}^{\left( \pm_{1}, \pm_{2}\right)}:=D_{\mathbf{A B C}}^{\left( \pm_{1}, \pm_{2}\right)}[F]
$$

and

$$
\begin{aligned}
R:= & R\left[\phi_{0}\right]+\sum_{\mathbf{A}}\left(R_{\mathbf{A}}[F]+R_{\mathbf{A}}[\widetilde{F}]+R_{\mathbf{A}}\left[\widetilde{F}^{(2)}\right]+R_{\mathbf{A}}\left[\widetilde{F}^{(3)}\right]\right) \\
& +\sum_{\mathbf{A}}\left(\lambda^{2}\left(g_{0}^{-1}\right)^{t t} \partial_{t}^{2} \widetilde{F}_{\mathbf{A}}-\left(\partial_{t}\left(g_{3}^{\prime}\right)^{t \beta}+\left(g_{0}^{-1}\right)^{t \beta} \frac{\partial_{t} N_{3}}{N_{0}}\right) \partial_{\beta} u_{\mathbf{A}} F_{\mathbf{A}}\right) .
\end{aligned}
$$

6.3. Estimates for $\widetilde{F}_{\mathbf{A}}$. We now begin to estimate the terms in the parametrix for $\phi$. Since $\widetilde{F}_{\mathbf{A}}, \widetilde{F}_{\mathbf{A}}^{(2)}$ and $\widetilde{F}_{\mathbf{A}}^{(3)}$ are defined via transport equations, it is convenient to have a general lemma for obtaining estimates:

Lemma 6.14. Suppose $f$ satisfies the transport equation

$$
2\left(g_{0}^{-1}\right)^{\alpha \beta}\left(\partial_{\alpha} u_{\mathbf{A}}\right) \partial_{\beta} f+\left(\square_{g_{0}} u_{\mathbf{A}}\right) f=h,
$$

with $f$ and $h$ bott 30 compactly supported in $B\left(0, R_{\text {supp }}\right)$. Then, the following estimate holds for $k \leq 9$ :

$$
\sup _{t \in[0,1]}\|f\|_{H^{k}}(t) \leq C\left(C_{0}\right)\left(\|f\|_{H^{k}}(0)+\int_{0}^{1}\|h\|_{H^{k}}(s) d s\right),
$$

the following estimate holds for $k \leq 8$ :

$$
\sup _{t \in[0,1]}\left\|\partial_{t} f\right\|_{H^{k}}(t) \leq C\left(C_{0}\right)\left(\|f\|_{H^{k+1}}(0)+\int_{0}^{1}\|h\|_{H^{k+1}}(s) d s\right),
$$

\footnotetext{
${ }^{30}$ Note the trivial fact that just assuming that $\operatorname{supp}(h) \subset B\left(0, R_{\text {supp }}\right)$ does not imply $\operatorname{supp}(f) \subset B\left(0, R_{\text {supp }}\right)$. This lemma however applies when the support properties are assumed for both $f$ and $h$.
} 
and the following estimate holds for $k \leq 7$ :

$$
\sup _{t \in[0,1]}\left\|\partial_{t}^{2} f\right\|_{H^{k}}(t) \leq C\left(C_{0}\right)\left(\left\|\partial_{t} f\right\|_{H^{k+1}}(0)+\int_{0}^{1}\left(\|h\|_{H^{k+1}}+\left\|\partial_{t} h\right\|_{H^{k+1}}\right)(s) d s\right) .
$$

Proof. In coordinates, we can write

$$
2\left(g_{0}^{-1}\right)^{\alpha t} \partial_{\alpha} u_{\mathbf{A}} \partial_{t} f+2\left(g_{0}^{-1}\right)^{i \alpha} \partial_{\alpha} u_{\mathbf{A}} \partial_{i} f+\left(\square_{g_{0}} u_{\mathbf{A}}\right) f=h
$$

To derive the desired estimate in the $k=0$ case, simply multiply (6.17) by $f$, integrate in a spacetime region $[0, t] \times \mathbb{R}^{2}$ for $t \in[0,1]$ with respect to the measure $d x d t$, integrate by parts and apply the obvious estimates. Notice that the compact support of $f$ and $h$ allows us not to track the behavior at infinity. For higher $k$, one simply differentiate the equation $k$ times in $x$. Notice that we need $k \leq 9$ due to the regularity of the background solution given in Corollary 3.5 .

Next, for the estimates on $\partial_{t} f$ (i.e., (6.15)), one uses the equation (6.17) to rewrite $\partial_{t} f$ as $\partial_{i} f$ plus lower order terms. This is possible since we have a lower bound on $\left|\left(g_{0}^{-1}\right)^{\alpha t} \partial_{\alpha} u_{\mathbf{A}}\right|$ thanks to the bound $\left|\nabla u_{\mathbf{A}}\right|>\frac{1}{4}$ (see Definition 4.6) and the fact that $u_{\mathbf{A}}$ is an eikonal function (with respect to $g_{0}$ ).

Finally, for (6.16), we simply differentiate (6.17) by $\partial_{t}$, apply (6.15) to $\tilde{f}=\partial_{t} f$ (with $\tilde{h}=\partial_{t} h+$ $\left.\left[2\left(g_{0}^{-1}\right)^{\alpha \beta}\left(\partial_{\alpha} u_{\mathbf{A}}\right) \partial_{\beta}, \partial_{t}\right] f-\left(\partial_{t} \square_{g_{0}} u_{\mathbf{A}}\right) f\right)$ and then use (6.14) and (6.15) to control the error terms.

Consequently, we have

Proposition 6.15. $\widetilde{F}_{\mathbf{A}}$ satisfies the following estimates:

$$
\sum_{k \leq 3}\left(\lambda^{k}\left\|\widetilde{F}_{\mathbf{A}}\right\|_{H^{2+k}}+\lambda^{k}\left\|\partial_{t} \widetilde{F}_{\mathbf{A}}\right\|_{H^{1+k}}+\lambda^{k+1}\left\|\partial_{t}^{2} \widetilde{F}_{\mathbf{A}}\right\|_{H^{k}}\right) \leq C\left(C_{0}\right) \varepsilon+C\left(C_{1}\right) \varepsilon^{2}
$$

Proof. The idea is to apply Lemma 6.14. In the transport equation (4.19) for $\widetilde{F}_{\mathbf{A}}$, except for the term $\frac{1}{\lambda^{2}} F_{\mathbf{A}}\left(g_{3}^{\prime}\right)^{\alpha \beta} \partial_{\alpha} u_{\mathbf{A}} \partial_{\beta} u_{\mathbf{A}}$, all the terms on the RHS depend only on the background and can be controlled in the norm $\sum_{k \leq 3} \lambda^{k}\|\cdot\|_{H^{2+k}}$ by $\leq C\left(C_{0}\right) \varepsilon$. (In particular, the $A_{\mathbf{A}}^{(1)}$ term can be controlled by Proposition 6.9]) It thus remains to control the term $\frac{1}{\lambda^{2}} F_{\mathbf{A}}\left(g_{3}^{\prime}\right)^{\alpha \beta} \partial_{\alpha} u_{\mathbf{A}} \partial_{\beta} u_{\mathbf{A}}$. The main contribution of this term comes from $g_{3}^{\prime}$, which can be estimated by Proposition 6.2 .

Notice that we have in particular improved the bootstrap assumption (BA1).

6.4. Estimates for the terms $\widetilde{F}_{\mathbf{A}}^{(2)}$ and $\widetilde{F}_{\mathbf{A}}^{(3)}$.

Proposition 6.16. For $a=2,3, \widetilde{F}_{\mathbf{A}}^{(a)}$ (which depends only on the background solution) obeys the following estimates

$$
\left\|\widetilde{F}_{\mathbf{A}}^{(a)}\right\|_{H^{8}}+\left\|\partial_{t} \widetilde{F}_{\mathbf{A}}^{(a)}\right\|_{H^{7}}+\left\|\partial_{t}^{2} \widetilde{F}_{\mathbf{A}}^{(a)}\right\|_{H^{6}} \leq C\left(C_{0}\right) \varepsilon^{2}
$$

Proof. By Lemma 6.14 and the transport equations (4.20) and (4.21), it suffices to control the terms $A_{\mathbf{A}}^{(a)}$ and $\partial_{t} A_{\mathbf{A}}^{(a)}$. This then follows from Proposition 6.9.

6.5. Estimates for the remaining term $\mathcal{E}_{\lambda}$. Given the estimates derived in Sections 6.3 and 6.4 above, in order to control the scalar field $\phi_{\lambda}$, it remains to estimate $\mathcal{E}_{\lambda}$. Since $\square_{g} \phi_{\lambda}=0$ by (1.3), $\mathcal{E}_{\lambda}$ obeys the equation

$$
\square_{g} \mathcal{E}_{\lambda}=-\square_{g}\left(\phi_{\lambda}-\mathcal{E}_{\lambda}\right)=-(\text { terms in Proposition } 6.13) .
$$

We further decompose $\mathcal{E}_{\lambda}$ and the remainder of this section will be dedicated to estimating each of the decomposed piece. More precisely, define

$$
\mathcal{E}_{\lambda}:=\mathcal{E}_{\lambda}^{(\text {elliptic })}+\mathcal{E}_{\lambda}^{\left(\partial_{t} \mathfrak{g}_{3}\right)}+\mathcal{E}_{\lambda}^{(\text {Error })}
$$

\footnotetext{
${ }^{31}$ In fact, if we carefully track the regularity of $\square_{g_{0}} u_{\mathbf{A}}$ in the proof of Theorem 3.3 we can have $k \leq 10$. Such improvement is of course completely irrelevant.
} 
where

$$
\begin{aligned}
& \mathcal{E}_{\lambda}^{(\text {elliptic })} \\
: & \sum_{ \pm} \sum_{\mathbf{A}} \sum_{\mathbf{B} \neq \mathbf{A}} \frac{\lambda^{3} B_{\mathbf{A B}}^{(2, \pm)}}{\left(g_{0}^{-1}\right)^{\alpha \beta}\left(\partial_{\alpha}\left(u_{\mathbf{A}} \pm u_{\mathbf{B}}\right)\right)\left(\partial_{\beta}\left(u_{\mathbf{A}} \pm u_{\mathbf{B}}\right)\right)} \sin \left(\frac{u_{\mathbf{A}} \pm u_{\mathbf{B}}}{\lambda}\right) \\
& +\sum_{ \pm} \sum_{\mathbf{A}} \sum_{\mathbf{B}} \frac{\lambda^{3} B_{\mathbf{A B}}^{(3, \pm)}}{\left(g_{0}^{-1}\right)^{\alpha \beta}\left(\partial_{\alpha}\left(u_{\mathbf{A}} \pm 2 u_{\mathbf{B}}\right)\right)\left(\partial_{\beta}\left(u_{\mathbf{A}} \pm 2 u_{\mathbf{B}}\right)\right)} \cos \left(\frac{u_{\mathbf{A}} \pm 2 u_{\mathbf{B}}}{\lambda}\right) \\
& +\sum_{ \pm_{1}} \sum_{ \pm_{2}} \sum_{\mathbf{A}} \sum_{\mathbf{B} \neq \mathbf{A}} \sum_{\substack{\mathbf{C} \neq \mathbf{B} \\
\mathbf{C} \neq \mathbf{A}}} \frac{\lambda^{3} D_{\mathbf{A B C}}^{\left( \pm_{1}, \pm_{2}\right)}}{\left(g_{0}^{-1}\right)^{\alpha \beta}\left(\partial_{\alpha}\left(u_{\mathbf{A}} \pm_{1} u_{\mathbf{B}} \pm_{2} u_{\mathbf{C}}\right)\right)\left(\partial_{\beta}\left(u_{\mathbf{A}} \pm_{1} u_{\mathbf{B}} \pm_{2} u_{\mathbf{C}}\right)\right)} \cos \left(\frac{u_{\mathbf{A}} \pm_{1} u_{\mathbf{B}} \pm_{2} u_{\mathbf{C}}}{\lambda}\right),
\end{aligned}
$$

with $B_{\mathrm{AB}}^{(2, \pm)}, B_{\mathrm{AB}}^{(3, \pm)}$ and $D_{\mathrm{ABC}}^{\left( \pm_{1}, \pm_{2}\right)}$ as in Proposition 6.13, $\mathcal{E}_{\lambda}^{\left(\partial_{t} \mathfrak{g}_{3}\right)}$ is the unique solution to the wave equation

$$
\left\{\begin{array}{l}
\square_{g} \mathcal{E}_{\lambda}^{\left(\partial_{t} \mathfrak{g}_{3}\right)}=-\left(\partial_{t}\left(g_{3}^{\prime}\right)^{t \beta}+\left(g_{0}^{-1}\right)^{t \beta} \frac{\partial_{t} N_{3}}{N_{0}}\right)\left(\partial_{\beta} \phi_{0}\right) \\
\left(\mathcal{E}_{\lambda}^{\left(\partial_{t} \mathfrak{g}_{3}\right)}, \partial_{t} \mathcal{E}_{\lambda}^{\left(\partial_{t} \mathfrak{g}_{3}\right)}\right)\left\lceil\Sigma_{0}=(0,0) ;\right.
\end{array}\right.
$$

and $\mathcal{E}_{\lambda}^{(\text {Error })}$ is defined so that (6.19) holds. According to Proposition6.13 this means that $\mathcal{E}_{\lambda}^{(\text {Error })}$ satisfies

$$
\begin{aligned}
-\square_{g} \mathcal{E}_{\lambda}^{(\text {Error })}= & R+\sum_{ \pm} \sum_{\mathbf{A}} \sum_{\mathbf{B} \neq \mathbf{A}} \lambda B_{\mathbf{A B}}^{(2, \pm)} \sin \left(\frac{u_{\mathbf{A}} \pm u_{\mathbf{B}}}{\lambda}\right)+\sum_{ \pm} \sum_{\mathbf{A}} \sum_{\mathbf{B}} \lambda B_{\mathbf{A B}}^{(3, \pm)} \cos \left(\frac{u_{\mathbf{A}} \pm 2 u_{\mathbf{B}}}{\lambda}\right) \\
& +\sum_{ \pm_{1}} \sum_{ \pm_{2}} \sum_{\mathbf{A}} \sum_{\mathbf{B} \neq \mathbf{A}} \sum_{\substack{\mathbf{C} \neq \mathbf{B} \\
\mathbf{C} \neq \mathbf{A}}} \lambda D_{\mathbf{A B C}}^{\left( \pm_{1}, \pm_{2}\right)} \cos \left(\frac{u_{\mathbf{A}} \pm_{1} u_{\mathbf{B}} \pm_{2} u_{\mathbf{C}}}{\lambda}\right)+\square_{g} \mathcal{E}_{\lambda}^{(\text {elliptic })}
\end{aligned}
$$

with initial conditions

$$
\begin{aligned}
& \mathcal{E}_{\lambda}^{(\text {Error })}\left\lceil\Sigma_{0}=\phi\right.-\phi_{0}-\sum_{\mathbf{A}} \lambda F_{\mathbf{A}} \cos \left(\frac{u_{\mathbf{A}}}{\lambda}\right)-\mathcal{E}_{\lambda}^{(\text {elliptic })} \\
&\left.\partial_{t} \mathcal{E}_{\lambda}^{(\text {Error })}\right|_{\Sigma_{0}=} \partial_{t}\left(\phi-\phi_{0}-\sum_{\mathbf{A}} \lambda F_{\mathbf{A}} \cos \left(\frac{u_{\mathbf{A}}}{\lambda}\right)-\sum_{\mathbf{A}} \lambda^{2} \widetilde{F}_{\mathbf{A}} \sin \left(\frac{u_{\mathbf{A}}}{\lambda}\right)\right. \\
&\left.\quad-\sum_{\mathbf{A}} \lambda^{2} \widetilde{F}_{\mathbf{A}}^{(2)} \cos \left(\frac{2 u_{\mathbf{A}}}{\lambda}\right)-\sum_{\mathbf{A}} \lambda^{2} \widetilde{F}_{\mathbf{A}}^{(3)} \sin \left(\frac{3 u_{\mathbf{A}}}{\lambda}\right)-\mathcal{E}_{\lambda}^{(\text {elliptic })}\right) \Gamma_{0} .
\end{aligned}
$$

Notice that $\mathcal{E}_{\lambda}^{(\text {elliptic) }}$ is well-defined thanks to the fact that the $u_{\mathbf{A}}$ 's are null adapted (i.e., by Definition 4.7 we can divide by $\left(g_{0}^{-1}\right)^{\alpha \beta}\left(\partial_{\alpha}\left(u_{\mathbf{A}} \pm u_{\mathbf{B}}\right)\right)\left(\partial_{\beta}\left(u_{\mathbf{A}} \pm u_{\mathbf{B}}\right)\right)$, etc.) The choice of $\mathcal{E}_{\lambda}^{(\text {elliptic) }}$ (and $\left.\mathcal{E}_{\lambda}^{\left(\partial_{t} \mathfrak{g}_{3}\right)}\right)$ is such that the RHS of (6.22) and the initial conditions in (6.23) are at most of size $C\left(C_{1}\right) \varepsilon \lambda^{2}$ and so that $\mathcal{E}_{\lambda}^{(\text {elliptic) }}$ itself is also at most $C\left(C_{0}\right) \varepsilon^{3} \lambda^{3}$. That this can be achieved is precisely because the $u_{\mathbf{A}}$ 's are null adapted so that order $C\left(C_{0}\right) \varepsilon^{2} \lambda$ oscillating terms are oscillating in a non-null direction. Consequently, for the high frequency part, $\square_{g}$ heuristically "looks like an elliptic operator".

In the next two propositions, we make precise the smallness of $\mathcal{E}_{\lambda}^{(\text {elliptic })}$, of the RHS of (6.22) and of (6.23).

Proposition 6.17. $\mathcal{E}_{\lambda}^{(\text {elliptic })}$ satisfies

$$
\sum_{k \leq 8} \lambda^{k}\left\|\mathcal{E}_{\lambda}^{(\text {elliptic) }}\right\|_{H^{k}}+\sum_{k \leq 7} \lambda^{k+1}\left\|\partial_{t} \mathcal{E}_{\lambda}^{(\text {elliptic })}\right\|_{H^{k}}+\sum_{k \leq 6} \lambda^{k+2}\left\|\partial_{t}^{2} \mathcal{E}_{\lambda}^{(\text {elliptic) }}\right\|_{H^{k}} \leq C\left(C_{0}\right) \varepsilon^{3} \lambda^{3} .
$$

Proof. This is an immediate consequence of the definition (6.20) and the estimates in Proposition 6.13 for $B_{\mathbf{A B}}^{(2, \pm)}, B_{\mathbf{A B}}^{(3, \pm)}$ and $D_{\mathbf{A B C}}^{\left( \pm_{1}, \pm_{2}\right)}$.

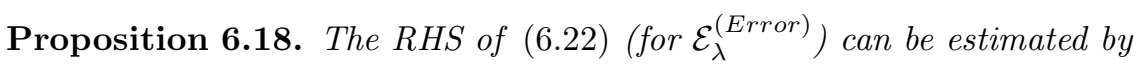

$$
\sum_{k \leq 3} \lambda^{k}\|\cdot\|_{H^{k}} \leq C\left(C_{1}\right) \varepsilon \lambda^{2} .
$$


Moreover, the initial data for $\mathcal{E}_{\lambda}^{(\text {Error })}$ can be bounded as follows:

$$
\sum_{k \leq 5} \lambda^{k} \| \mathcal{E}_{\lambda}^{(\text {Error })}\left\lceil_{\Sigma_{0}}\left\|_{H^{k}}+\sum_{k \leq 4} \lambda^{k+1}\right\|\left(\partial_{t} \mathcal{E}_{\lambda}^{(\text {Error })}\right) \Sigma_{\Sigma_{0}} \|_{H^{k}} \leq C\left(C_{0}\right) \varepsilon \lambda^{2} .\right.
$$

Proof. To estimate the RHS of (6.22), we first note that the term $R$ satisfies the desired estimate by (6.13) in Proposition 6.13. Then, note that $\mathcal{E}_{\lambda}^{(\text {elliptic) }}$ is constructed so that $\square_{g} \mathcal{E}_{\lambda}^{(\text {elliptic) }}$ cancels with the remaining terms up to error terms of size $C\left(C_{0}\right) \varepsilon^{3} \lambda^{2}$, i.e.,

$$
\begin{aligned}
& \sum_{k \leq 6} \lambda^{k} \| \sum_{ \pm} \sum_{\mathbf{A}} \sum_{\mathbf{B} \neq \mathbf{A}} \lambda B_{\mathbf{A B}}^{(2, \pm)} \sin \left(\frac{u_{\mathbf{A}} \pm u_{\mathbf{B}}}{\lambda}\right)+\sum_{ \pm} \sum_{\mathbf{A}} \sum_{\mathbf{B}} \lambda B_{\mathbf{A B}}^{(3, \pm)} \cos \left(\frac{u_{\mathbf{A}} \pm 2 u_{\mathbf{B}}}{\lambda}\right) \\
& +\sum_{ \pm_{1}} \sum_{ \pm_{2}} \sum_{\mathbf{A}} \sum_{\mathbf{B} \neq \mathbf{A}} \sum_{\substack{\mathbf{C} \neq \mathbf{B} \\
\mathbf{C} \neq \mathbf{A}}} \lambda D_{\mathbf{A B C}}^{\left( \pm_{1}, \pm_{2}\right)} \cos \left(\frac{u_{\mathbf{A}} \pm_{1} u_{\mathbf{B}} \pm_{2} u_{\mathbf{C}}}{\lambda}\right)-\square_{g} \mathcal{E}_{\lambda}^{(\text {elliptic) }} \|_{H^{k}} \leq C\left(C_{0}\right) \varepsilon^{3} \lambda^{2} .
\end{aligned}
$$

We hence conclude (6.24). To prove (6.25), we recall (6.23). By Lemma 4.9,

$$
\left\|\left(\phi-\phi_{0}-\sum_{\mathbf{A}} \lambda F_{\mathbf{A}} \cos \left(\frac{u_{\mathbf{A}}}{\lambda}\right), \dot{\phi}-\dot{\phi}_{0}+\sum_{\mathbf{A}} F_{\mathbf{A}}\left|\nabla u_{\mathbf{A}}\right| e^{\gamma_{0}} \sin \left(\frac{u_{\mathbf{A}}}{\lambda}\right)\right) \Sigma_{\Sigma_{0}}\right\|_{H^{5} \times H^{4}} \leq C \varepsilon \lambda^{2} .
$$

By Proposition 6.17

$$
\sum_{k \leq 8} \lambda^{k}\left\|\mathcal{E}_{\lambda}^{(\text {elliptic) }}\right\|_{H^{k}}+\sum_{k \leq 7} \lambda^{k+1}\left\|\partial_{t} \mathcal{E}_{\lambda}^{(\text {elliptic) }}\right\|_{H^{k}} \leq C\left(C_{0}\right) \varepsilon^{3} \lambda^{3}
$$

Therefore the initial data for $\left(\mathcal{E}_{\lambda}^{(\text {Error })}, \partial_{t} \mathcal{E}_{\lambda}^{(\text {Error })}\right)$ obey the stated estimates. (Here, we have used that the initial data for $\widetilde{F}_{\mathbf{A}}, \widetilde{F}_{\mathbf{A}}^{(2)}$ and $\widetilde{F}_{\mathbf{A}}^{(3)}$ are zero, and if a time derivative falls on these term (instead of the oscillating factors), this give a contribution that can be controlled by a higher power of $\lambda$ ).

Proposition 6.18 is already a good indication that $\mathcal{E}_{\lambda}^{(\text {Error })}$ can be appropriately controlled. To proceed, we consider general energy estimates for solutions to general wave equations so that we can treat both $\mathcal{E}_{\lambda}^{\left(\partial_{t} \mathfrak{g}_{3}\right)}$ and $\mathcal{E}_{\lambda}^{(\text {Error })}$.

Proposition 6.19. Let $f$ be a solution to

$$
\square_{g} f=h
$$

with both $f$ and $h$ being compactly supported in $B\left(0, R_{\text {supp }}\right)$. Then there exists a constant $C\left(C_{0}\right)>0$ such that $f$ obeys the estimate

$$
\sup _{t \in[0,1]}\|\partial f\|_{L^{2}}^{2}(t) \leq C\left(C_{0}\right)\left(\|\partial f\|_{L^{2}}^{2}(0)+\sup _{t \in[0,1]}\left|\int_{0}^{t} \int_{\Sigma_{t^{\prime}}}\left(\partial_{t} f\right) h\left(t^{\prime}, x\right) \sqrt{|\operatorname{det} g|} d x d t^{\prime}\right|\right) .
$$

Consequently, it also holds that

$$
\sup _{t \in[0,1]}\|\partial f\|_{L^{2}}(t) \leq C\left(C_{0}\right)\left(\|\partial f\|_{L^{2}}(0)+\|h\|_{L^{2}}\right) .
$$

Proof. Define a 2-tensor $Q$ as follows:

$$
Q_{\alpha \beta}[f]:=\partial_{\alpha} f \partial_{\beta} f-\frac{1}{2} g_{\alpha \beta}\left(g^{-1}\right)^{\rho \sigma} \partial_{\sigma} f \partial_{\rho} f .
$$

By the equation $\square_{g} f=h$, we have

$$
\left(g^{-1}\right)^{\mu \alpha} D_{\mu} Q_{\alpha \beta}[f]=h \partial_{\beta} f,
$$

where $D$ is the Levi-Civita connection associated to $g$. Define also the deformation tensor associated to $\partial_{t}$

$$
{ }^{\left(\partial_{t}\right)} \pi_{\alpha \beta}=D_{\alpha}\left(\partial_{t}\right)_{\beta}+D_{\beta}\left(\partial_{t}\right)_{\alpha} .
$$

By the Stoke's theorem, we have that for every $t \in(0,1]$,

$$
\begin{aligned}
& \int_{\Sigma_{t}} Q[f]\left(\partial_{t}, \frac{1}{N} e_{0}\right)(t, x) \sqrt{|\operatorname{det} \bar{g}|} d x \\
= & \int_{\Sigma_{0}} Q[f]\left(\partial_{t}, \frac{1}{N} e_{0}\right)(0, x) \sqrt{|\operatorname{det} \bar{g}|} d x \\
& -\int_{0}^{t} \int_{\Sigma_{t^{\prime}}}\left(\left(\partial_{t} f\right) h+\frac{1}{2} Q_{\alpha \beta}[f]^{\left(\partial_{t}\right)} \pi^{\alpha \beta}\right)\left(t^{\prime}, x\right) \sqrt{|\operatorname{det} g|} d x d t^{\prime},
\end{aligned}
$$


where $\bar{g}$ is as in (3.1). It follows from our assumptions on the background and (B2), (BA3), (BA4) that on the compact set $B\left(0, R_{\text {supp }}\right)$,

$$
Q[f]\left(\partial_{t}, \frac{1}{N} e_{0}\right) \sim_{C_{0}}|\partial f|^{2}, \quad \sqrt{|\operatorname{det} \bar{g}|} \sim_{C_{0}} \sqrt{|\operatorname{det} g|} \sim_{C_{0}} 1, \quad\left|Q_{\alpha \beta}[f]^{\left(\partial_{t}\right)} \pi^{\alpha \beta}\right| \leq C\left(C_{0}\right)|\partial f|^{2},
$$

where we denote $A \sim \sim_{C_{0}} B$ if $A \leq C\left(C_{0}\right) B$ and $B \leq C\left(C_{0}\right) A$. (6.26) hence follows from the above observations and Grönwall's inequality. To get from (6.26) to (6.27), one simply applies the Cauchy-Schwarz inequality.

From Proposition 6.19, we also derive the following energy estimates for higher derivatives:

Corollary 6.20. Let $f$ and $h$ be as in Proposition 6.19. For $1 \leq k \leq 3$, it holds that

$$
\begin{aligned}
& \sup _{t \in[0,1]}\left(\|\partial f\|_{H^{k}}(t)+\left\|\partial^{2} f\right\|_{H^{k-1}}(t)\right) \\
\leq & C\left(C_{0}\right)\left(\|\partial f\|_{H^{k}}(0)+\|h\|_{H^{k}}+\sum_{k^{\prime} \leq k-1} \lambda^{k^{\prime}-k}\left(\|\partial f\|_{H^{k^{\prime}}}+\left\|\partial^{2} f\right\|_{H^{k^{\prime}-1}}\right)\right) .
\end{aligned}
$$

Proof. We first estimate $\|\partial f\|_{H^{k}}$. The idea is to apply (6.27) for $\tilde{f}=\partial_{x^{1}}^{k_{1}} \partial_{x^{2}}^{k_{2}} f$ and then sum over all the estimates for $k_{1}+k_{2} \leq k$. For such $\tilde{f}$, we have

$$
\square_{g} \tilde{f}=\tilde{h}:=\partial_{x^{1}}^{k_{1}} \partial_{x^{2}}^{k_{2}} h+\left[\square_{g}, \partial_{x^{1}}^{k_{1}} \partial_{x^{2}}^{k_{2}}\right] f .
$$

Now for $k_{1}+k_{2} \leq k$, we use Corollary 3.5 to control the background metric and obtain

$$
\begin{aligned}
& \left|\left[\square_{g}, \partial_{x^{1}}^{k_{1}} \partial_{x^{2}}^{k_{2}}\right] f\right| \\
& \leq C\left(C_{0}\right)(\underbrace{\sum_{k_{1}^{\prime} \leq k} \varepsilon\left|\partial \nabla^{k_{1}^{\prime}} f\right|}_{=: I_{k}}+\underbrace{\sum_{k_{1}^{\prime} \leq k-1} \varepsilon\left|\partial^{2} \nabla^{k_{1}^{\prime}} f\right|}_{=: I I_{k}} \\
& +\underbrace{\sum_{i=1,2,3} \sum_{k_{1}^{\prime}+k_{2}^{\prime} \leq k}\left|\partial \nabla^{k_{1}^{\prime}} \mathfrak{g}_{i}\right|\left|\partial \nabla^{k_{2}^{\prime}} f\right|}_{=: I I I_{k}}+\underbrace{\sum_{i=1,2,3} \sum_{\substack{k_{1}^{\prime}+k_{2}^{\prime} \leq k \\
k_{1}^{\prime} \geq 1}}\left|\nabla^{k_{1}^{\prime}} \mathfrak{g}_{i}\right|\left|\partial^{2} \nabla^{k_{2}^{\prime}} f\right|}_{=: I V_{k}}) .
\end{aligned}
$$

Therefore, applying (6.27) to $\tilde{f}$ (for every $k_{1}+k_{2} \leq k$ ) and using (6.29) and (6.30), we get

$$
\sup _{t \in[0,1]}\|\partial f\|_{H^{k}}(t) \leq C\left(C_{0}\right)\left(\|\partial f\|_{H^{k}}(0)+\|h\|_{H^{k}}+\left\|I_{k}\right\|_{L^{2}}+\left\|I I_{k}\right\|_{L^{2}}+\left\|I I I_{k}\right\|_{L^{2}}+\left\|I V_{k}\right\|_{L^{2}}\right)
$$

It therefore remains to control the terms $I_{k}, I I_{k}, I I I_{k}$ and $I V_{k}$. It is immediate that

$$
\begin{array}{r}
\left\|I_{k}\right\|_{L^{2}} \leq C\left(C_{0}\right)\|\partial f\|_{H^{k}} \\
\left\|I I_{k}\right\|_{L^{2}} \leq C\left(C_{0}\right)\left\|\partial^{2} f\right\|_{H^{k-1}}
\end{array}
$$

For $I I I_{k}$, we split into the case where $k \leq 2$ and $k=3$. If $\underline{k \leq 2}$, then by Hölder's inequality, (B2), (5.7) and (5.8),

$$
\left\|I I I_{k}\right\|_{L^{2}} \leq C\left(C_{1}\right) \varepsilon \sum_{\substack{k_{1}^{\prime}+k_{2}^{\prime} \leq k \\ k_{1}^{\prime} \leq 2}} \lambda^{-k_{1}^{\prime}}\|\partial f\|_{H^{k_{2}^{\prime}}} .
$$

If $\underline{k=3}$ and $i=1$, it can be treated as in the $k \leq 2$ case. If $\underline{k=3}$ and $i \neq 1$, we treat separately the cases $k_{1}^{\prime} \leq 2$ and $k_{1}^{\prime}=3$ using Hölder's inequality, (BA3), (BA4), (5.7) and (5.8) to get

$$
\begin{aligned}
\left\|I I I_{k}\right\|_{L^{2}} & \leq C\left(C_{1}\right) \varepsilon \sum_{\substack{k_{1}^{\prime}+k_{2}^{\prime} \leq k \\
k_{1}^{\prime} \leq 2}} \lambda^{-k_{1}^{\prime}}\|\partial f\|_{H^{k_{2}^{\prime}}}+\sum_{i=2,3}\left\|\partial \mathfrak{g}_{i}\right\|_{H^{3}}\|\partial f\|_{L^{\infty}} \\
& \leq C\left(C_{1}\right) \varepsilon \sum_{k_{1}^{\prime}+k_{2}^{\prime} \leq 3} \lambda^{-k_{1}^{\prime}}\|\partial f\|_{H^{k_{2}^{\prime}}}+C\left(C_{1}\right) \varepsilon \lambda^{-1}\|\partial f\|_{H^{2}} \leq C\left(C_{1}\right) \varepsilon \sum_{k_{1}^{\prime}+k_{2}^{\prime} \leq k} \lambda^{-k_{1}^{\prime}}\|\partial f\|_{H^{k_{2}^{\prime}}},
\end{aligned}
$$


where we have also used Proposition A.4 (more specifically $H^{2} \subset L^{\infty}$ ) for $\partial f$. Hence, in both cases, $\left\|I I I_{k}\right\|_{L^{2}}$ obeys the desired estimate. Finally, for $I V_{k}$, using (B2), (5.7) and (5.8), we get

$$
\left\|I V_{k}\right\|_{L^{2}} \leq C\left(C_{1}\right) \varepsilon \sum_{\substack{k_{1}^{\prime}+k_{2}^{\prime} \leq k \\ k_{1}^{\prime} \geq 1}} \lambda^{1-k_{1}^{\prime}}\left\|\partial^{2} f\right\|_{H_{2}^{k_{2}^{\prime}}} .
$$

Combining (6.31), (6.32), (6.33), (6.34), (6.35) and (6.36), we obtain

$$
\begin{aligned}
& \sup _{t \in[0,1]}\|\partial f\|_{H^{k}}(t) \\
\leq C\left(C_{0}\right) & \left(\|\partial f\|_{H^{k}}(0)+\|h\|_{H^{k}}+C\left(C_{1}\right) \varepsilon\left(\sum_{k_{1}^{\prime}+k_{2}^{\prime} \leq k} \lambda^{-k_{1}^{\prime}}\|\partial f\|_{H_{2}^{k_{2}^{\prime}}}+\sum_{\substack{k_{1}^{\prime}+k_{2}^{\prime} \leq k \\
k_{1}^{\prime} \geq 1}} \lambda^{1-k_{1}^{\prime}}\left\|\partial^{2} f\right\|_{H^{k_{2}^{\prime}}}\right)\right) .
\end{aligned}
$$

The estimate (6.37) almost controls all components of $\left\|\partial^{2} f\right\|_{H^{k-1}}(t)$, except for the term $\left\|\partial_{t}^{2} f\right\|_{H^{k-1}}(t)$. Now using the wave equation and noticing that $\left(g^{-1}\right)^{t t}$ is bounded away from 0 on $B\left(0, R_{\text {supp }}\right)$, we can write

to get

$$
\partial_{t}^{2} f=-\frac{1}{\left(g^{-1}\right)^{t t}}\left(2\left(g^{-1}\right)^{t i} \partial_{t i}^{2} f+\left(g^{-1}\right)^{i j} \partial_{i j}^{2} f-\left(g^{-1}\right)^{\alpha \beta} \Gamma_{\alpha \beta}^{\rho} \partial_{\rho} f-h\right)
$$

$$
\begin{aligned}
& \sup _{t \in[0,1]}\left(\|\partial f\|_{H^{k}}(t)+\left\|\partial^{2} f\right\|_{H^{k-1}}(t)\right) \\
\leq C\left(C_{0}\right) & \left(\|\partial f\|_{H^{k}}(0)+\|h\|_{H^{k}}+C\left(C_{1}\right) \varepsilon\left(\sum_{k_{1}^{\prime}+k_{2}^{\prime} \leq k} \lambda^{-k_{1}^{\prime}}\|\partial f\|_{H_{2}^{k_{2}^{\prime}}}+\sum_{\substack{k_{1}^{\prime}+k_{2}^{\prime} \leq k \\
k_{1}^{\prime} \geq 1}} \lambda^{1-k_{1}^{\prime}}\left\|\partial^{2} f\right\|_{H^{k_{2}^{\prime}}}\right)\right) .
\end{aligned}
$$

The conclusion follows after absorbing $C\left(C_{1}\right) \varepsilon\left(\|\partial f\|_{H^{k}}(t)+\left\|\partial^{2} f\right\|_{H^{k-1}}(t)\right)$ to the LHS.

Proposition 6.21. $\mathcal{E}_{\lambda}^{\left(\partial_{t} \mathfrak{g}_{3}\right)}$ satisfies

$$
\sum_{k \leq 3} \lambda^{k}\left\|\partial \mathcal{E}_{\lambda}^{\left(\partial_{t} \mathfrak{g}_{3}\right)}\right\|_{H^{k}}+\sum_{k \leq 2} \lambda^{k+1}\left\|\partial^{2} \mathcal{E}_{\lambda}^{\left(\partial_{t} \mathfrak{g}_{3}\right)}\right\|_{H^{k}} \leq C\left(C_{1}\right) \varepsilon \lambda^{2}
$$

Proof. The idea is of course to apply Proposition 6.19 (more precisely, 6.26$)$ ) and Corollary 6.20 for

$$
f=\mathcal{E}_{\lambda}^{\left(\partial_{t} \mathfrak{g}_{3}\right)} \text { and } h=-\left(\partial_{t}\left(g_{3}^{\prime}\right)^{t \beta}+\left(g_{0}^{-1}\right)^{t \beta} \frac{\partial_{t} N_{3}}{N_{0}}\right)\left(\partial_{\beta} \phi_{0}\right) .
$$

We will do this in the following steps:

Estimates for $\left\|\partial \mathcal{E}_{\lambda}^{\left(\partial_{t} \mathfrak{g}_{3}\right)}\right\|_{L^{2}}$. It turns out that this is the hardest case in this proposition. By the assumptions on the background solution and the bootstrap assumption (BA4), we have $\|h\|_{L^{2}} \leq C\left(C_{1}\right) \varepsilon \lambda$, which if we directly apply (6.27) gives an estimate that is off by one factor of $\lambda^{-1}$. Instead, we use (6.26). To simplify the exposition, let us define $h=h_{1}+h_{2}$ with $h_{1}:=\left(\partial_{t}\left(g_{3}^{\prime}\right)^{t \beta}\right)\left(\partial_{\beta} \phi_{0}\right)$ and $h_{2}:=\left(g_{0}^{-1}\right)^{t \beta} \frac{\partial_{t} N_{3}}{N_{0}}\left(\partial_{\beta} \phi_{0}\right)$. Since they can be treated similarly, we will only consider $h_{1}$. More precisely, we consider the following term that arises in (6.26):

$$
\sup _{t \in[0,1]}\left|\int_{0}^{t} \int_{\Sigma_{t^{\prime}}}\left(\partial_{t} \mathcal{E}_{\lambda}^{\left(\partial_{t} \mathfrak{g}_{3}\right)}\right)\left(\partial_{t}\left(g_{3}^{\prime}\right)^{t \beta}\right)\left(\partial_{\beta} \phi_{0}\right)\left(t^{\prime}, x\right) \sqrt{|\operatorname{det} g|} d x d t^{\prime}\right| .
$$

The idea is to integrate by parts in $t$, use the wave equation for $\mathcal{E}_{\lambda}^{\left(\partial_{t} \mathfrak{g}_{3}\right)}$ and integrate by parts in $x$. We integrate by parts below, but only writing down the main terms. The lower order terms (for instance when the derivatives fall on either $\phi_{0}$ or $\sqrt{|\operatorname{det} g|}$ or from the $\partial \mathcal{E}_{\lambda}^{\left(\partial_{t} \mathfrak{g}_{3}\right)}$ term in the wave equation) are bounded by $C\left(C_{1}\right) \varepsilon^{2} \lambda^{4}$ using (B2), (BA2), (BA3), (BA4), (5.7), (5.8) and the estimates for the background solution 32

\footnotetext{
${ }^{32}$ In particular, these lower order terms can be bounded with a factor of $C\left(C_{1}\right) \varepsilon$ from $\phi_{0}$ (and its derivatives), a factor of $C\left(C_{1}\right) \varepsilon \lambda^{2}$ from $g_{3}^{\prime}$ and a factor of $C\left(C_{1}\right) \lambda^{2}$ from $\partial \mathcal{E}$.
} 
in Corollary 3.5

$$
\begin{aligned}
& \sup _{t \in[0,1]}\left|\int_{0}^{t} \int_{\Sigma_{t^{\prime}}}\left(\partial_{t} \mathcal{E}_{\lambda}^{\left(\partial_{t} \mathfrak{g}_{3}\right)}\right)\left(\partial_{t}\left(g_{3}^{\prime}\right)^{t \beta}\right)\left(\partial_{\beta} \phi_{0}\right)\left(t^{\prime}, x\right) \sqrt{|\operatorname{det} g|} d x d t^{\prime}\right| \\
\leq & \sup _{t \in[0,1]}\left|\int_{0}^{t} \int_{\Sigma_{t^{\prime}}}\left(\partial_{t}^{2} \mathcal{E}_{\lambda}^{\left(\partial_{t} \mathfrak{g}_{3}\right)}\right)\left(g_{3}^{\prime}\right)^{t \beta}\left(\partial_{\beta} \phi_{0}\right)\left(t^{\prime}, x\right) \sqrt{|\operatorname{det} g|} d x d t^{\prime}\right| \\
& +2 \sup _{t \in[0,1]}\left|\int_{\Sigma_{t}}\left(\partial_{t} \mathcal{E}_{\lambda}^{\left(\partial_{t} \mathfrak{g}_{3}\right)}\right)\left(g_{3}^{\prime}\right)^{t \beta}\left(\partial_{\beta} \phi_{0}\right)(t, x) \sqrt{|\operatorname{det} g|} d x\right|+C\left(C_{1}\right) \varepsilon^{2} \lambda^{4} \\
\leq & \sup _{t \in[0,1]}\left|\int_{0}^{t} \int_{\Sigma_{t^{\prime}}} \frac{1}{\left(g^{-1}\right)^{t t}}\left(2\left(g^{-1}\right)^{t i} \partial_{i t}^{2} \mathcal{E}_{\lambda}^{\left(\partial_{t} \mathfrak{g}_{3}\right)}+\left(g^{-1}\right)^{i j} \partial_{i j}^{2} \mathcal{E}_{\lambda}^{\left(\partial_{t} \mathfrak{g}_{3}\right)}+h\right)\left(g_{3}^{\prime}\right)^{t \beta}\left(\partial_{\beta} \phi_{0}\right)\left(t^{\prime}, x\right) \sqrt{|\operatorname{det} g|} d x d t^{\prime}\right| \\
& +2 \sup _{t \in[0,1]}\left|\int_{\Sigma_{t}}\left(\partial_{t} \mathcal{E}_{\lambda}^{\left(\partial_{t} \mathfrak{g}_{3}\right)}\right)\left(g_{3}^{\prime}\right)^{t \beta}\left(\partial_{\beta} \phi_{0}\right)(t, x) \sqrt{|\operatorname{det} g|} d x\right|+C\left(C_{1}\right) \varepsilon^{2} \lambda^{4} \\
\leq & C\left(C_{0}\right) \varepsilon\left\|\partial \mathcal{E}_{\lambda}^{\left(\partial_{t} \mathfrak{g}_{3}\right)}\right\|_{L^{2}}\left(\left\|\nabla \mathfrak{g}_{3}\right\|_{L^{2}}+\left\|\mathfrak{g}_{3}\right\|_{L^{2}}\right)+C\left(C_{1}\right) \varepsilon^{2} \lambda^{4} \\
\leq & C\left(C_{1}\right) \varepsilon^{2} \lambda^{4},
\end{aligned}
$$

where in the last line we have again used (BA2) and (BA4).

Now, $h_{2}$ can be bounded in a similar fashion By (6.26) in Proposition 6.19, since the initial data vanish, we have

which then implies after taking square root that

$$
\left\|\partial \mathcal{E}_{\lambda}^{\left(\partial_{t} \mathfrak{g}_{3}\right)}\right\|_{L^{2}}^{2} \leq C\left(C_{1}\right) \varepsilon^{2} \lambda^{4}
$$

$$
\left\|\partial \mathcal{E}_{\lambda}^{\left(\partial_{t} \mathfrak{g}_{3}\right)}\right\|_{L^{2}} \leq C\left(C_{1}\right) \varepsilon \lambda^{2} .
$$

Estimates for higher derivatives. By the assumptions on the background solution and the bootstrap assumption (BA4), we have

$$
\sum_{k \leq 2}\|h\|_{H^{k}} \leq C\left(C_{1}\right) \varepsilon \lambda, \quad\|h\|_{H^{3}} \leq C\left(C_{1}\right) \varepsilon .
$$

Since the initial data vanish, applying Corollary 6.20 inductively in $k$ and using (6.38), we obtain the bound

$$
\sum_{1 \leq k \leq 3} \lambda^{k}\left\|\partial \mathcal{E}_{\lambda}^{\left(\partial_{t} \mathfrak{g}_{3}\right)}\right\|_{H^{k}}+\sum_{k \leq 2} \lambda^{k+1}\left\|\partial^{2} \mathcal{E}_{\lambda}^{\left(\partial_{t} \mathfrak{g}_{3}\right)}\right\|_{H^{k}} \leq C\left(C_{1}\right) \varepsilon \lambda^{2}
$$

This concludes the proof of the proposition.

Finally, we prove the following bounds for $\mathcal{E}_{\lambda}^{(\text {Error) }}$ :

Proposition 6.22. $\mathcal{E}_{\lambda}^{(\text {Error })}$ satisfies

$$
\sum_{k \leq 3} \lambda^{k}\left\|\partial \mathcal{E}_{\lambda}^{(\text {Error })}\right\|_{H^{k}}+\sum_{k \leq 2} \lambda^{k+1}\left\|\partial^{2} \mathcal{E}_{\lambda}^{(\text {Error })}\right\|_{H^{k}} \leq C\left(C_{0}\right) \varepsilon \lambda^{2} .
$$

Proof. This is an immediate consequence of Proposition 6.19 (more precisely, (6.27)), Corollary 6.20 and Proposition 6.18.

Finally, we combine all the estimates in this subsection in the following corollary:

Corollary 6.23. Each of $\mathcal{E}_{\lambda}^{(\text {elliptic })}, \mathcal{E}_{\lambda}^{\left(\partial_{t} \mathfrak{g}_{3}\right)}$ and $\mathcal{E}_{\lambda}^{(\text {Error })}$ satisfies

$$
\sum_{k \leq 3} \lambda^{k}\left\|\partial \mathcal{E}_{\lambda}^{(\cdot)}\right\|_{H^{k}}+\sum_{k \leq 2} \lambda^{k+1}\left\|\partial^{2} \mathcal{E}_{\lambda}^{(\cdot)}\right\|_{H^{k}} \leq C\left(C_{1}\right) \varepsilon \lambda^{2} .
$$

Consequently, we also have

$$
\sum_{k \leq 3} \lambda^{k}\left\|\partial \mathcal{E}_{\lambda}\right\|_{H^{k}}+\sum_{k \leq 2} \lambda^{k+1}\left\|\partial^{2} \mathcal{E}_{\lambda}\right\|_{H^{k}} \leq C\left(C_{1}\right) \varepsilon \lambda^{2} .
$$

Proof. This is a consequence of Propositions 6.17 6.21 and 6.22.

In particular, we have improved the bootstrap assumption (BA2). 


\section{ESTIMATES FOR THE METRIC COMPONENTS}

7.1. Calculations of the main terms. To begin, we consider the difference of the squares of the derivatives of the scalar field and collect the $O_{\varepsilon}(1)$ terms as well as the $O_{\varepsilon}(\lambda)$ terms:

Proposition 7.1. The difference $\left(\partial_{\mu} \phi\right)\left(\partial_{\nu} \phi\right)-\left(\partial_{\mu} \phi_{0}\right)\left(\partial_{\nu} \phi_{0}\right)$ can be expanded as follows:

$$
\left(\partial_{\mu} \phi\right)\left(\partial_{\nu} \phi\right)-\left(\partial_{\mu} \phi_{0}\right)\left(\partial_{\nu} \phi_{0}\right)=\Theta_{\mu \nu}^{(m a i n)}+\Theta_{\mu \nu}^{(1)}+\Theta_{\mu \nu}^{(2)}+\Theta_{\mu \nu}^{(\text {remainder })}
$$

where

$$
\Theta_{\mu \nu}^{(\operatorname{main})}:=\frac{1}{2} \sum_{\mathbf{A}} F_{\mathbf{A}}^{2}\left(\partial_{\mu} u_{\mathbf{A}}\right)\left(\partial_{\nu} u_{\mathbf{A}}\right)
$$

and

$$
\begin{aligned}
\Theta_{\mu \nu}^{(1)}:= & \underbrace{-2 \sum_{\mathbf{A}} F_{\mathbf{A}} \partial_{(\mu} \phi_{0} \partial_{\nu)} u_{\mathbf{A}} \sin \left(\frac{u_{\mathbf{A}}}{\lambda}\right)}_{\mathfrak{G}_{1,1}}+\underbrace{\frac{1}{2} \sum_{\mathbf{A}} F_{\mathbf{A}}^{2} \partial_{\mu} u_{\mathbf{A}} \partial_{\nu} u_{\mathbf{A}} \cos \left(\frac{2 u_{\mathbf{A}}}{\lambda}\right)}_{\mathfrak{G}_{1,2}} \\
& +\underbrace{\frac{1}{2} \sum_{ \pm} \sum_{\mathbf{A}} \sum_{\mathbf{B} \neq \mathbf{A}}(\mp 1) \cdot F_{\mathbf{A}} F_{\mathbf{B}} \partial_{\mu} u_{\mathbf{A}} \partial_{\nu} u_{\mathbf{B}} \cos \left(\frac{u_{\mathbf{A}} \pm u_{\mathbf{B}}}{\lambda}\right)}_{\mathfrak{G}_{1,3}},
\end{aligned}
$$

and

$$
\begin{aligned}
& \Theta_{\mu \nu}^{(2)}:=\underbrace{-\lambda \sum_{ \pm} \sum_{\mathbf{A}} \sum_{\mathbf{B} \neq \mathbf{A}} \partial_{(\mu} F_{\mathbf{A}} \partial_{\nu)} u_{\mathbf{B}} F_{\mathbf{B}} \sin \left(\frac{u_{\mathbf{A}} \pm u_{\mathbf{B}}}{\lambda}\right)}_{=: \mathfrak{G}_{2,1}} \\
& \underbrace{-\lambda \sum_{\mathbf{A}} \partial_{(\mu} F_{\mathbf{A}} \partial_{\nu)} u_{\mathbf{A}} F_{\mathbf{A}} \sin \left(\frac{2 u_{\mathbf{A}}}{\lambda}\right)}_{=: \mathfrak{G}_{2,2}} \\
& +\underbrace{2 \lambda \sum_{\mathbf{A}} \partial_{(\mu} \phi_{0} \partial_{\nu)} u_{\mathbf{A}}\left(\widetilde{F}_{\mathbf{A}} \cos \left(\frac{u_{\mathbf{A}}}{\lambda}\right)-2 \widetilde{F}_{\mathbf{A}}^{(2)} \sin \left(\frac{2 u_{\mathbf{A}}}{\lambda}\right)+3 \widetilde{F}_{\mathbf{A}}^{(3)} \cos \left(\frac{3 u_{\mathbf{A}}}{\lambda}\right)\right)}_{=: \mathfrak{G}_{2,3}} \\
& +\underbrace{2 \lambda \sum_{\mathbf{A}} \partial_{(\mu} \phi_{0} \partial_{\nu)} F_{\mathbf{A}} \cos \left(\frac{u_{\mathbf{A}}}{\lambda}\right)}_{=: \mathfrak{G}_{2,4}} \\
& \underbrace{-2 \lambda \sum_{\mathbf{A}, \mathbf{B}} \partial_{(\mu} u_{\mathbf{A}} \partial_{\nu)} u_{\mathbf{B}} F_{\mathbf{A}} \sin \left(\frac{u_{\mathbf{A}}}{\lambda}\right)\left(\widetilde{F}_{\mathbf{B}} \cos \left(\frac{u_{\mathbf{B}}}{\lambda}\right)-2 \widetilde{F}_{\mathbf{B}}^{(2)} \sin \left(\frac{2 u_{\mathbf{B}}}{\lambda}\right)+3 \widetilde{F}_{\mathbf{B}}^{(3)} \cos \left(\frac{3 u_{\mathbf{B}}}{\lambda}\right)\right)}_{=: \mathfrak{G}_{2,5}} .
\end{aligned}
$$

and the remainder term satisfies

$$
\sum_{k \leq 3} \lambda^{k}\left\|\Theta^{(\text {remainder })}\right\|_{H^{k}}+\sum_{k \leq 2} \lambda^{k+1}\left\|\partial_{t} \Theta^{(\text {remainder })}\right\|_{H^{k}} \leq C\left(C_{1}\right) \varepsilon^{2} \lambda^{2} .
$$

Proof. This is a direct computation using the parametrix (4.18) for $\phi$ and estimating the resulting terms with (B1), (BA1) and Corollary 6.23. In order to simplify the exposition, let us only check the bound for $\sum_{k<3} \lambda^{k}\left\|\Theta^{(\text {remainder })}\right\|_{H^{k}}$ in (17.5) in the proof. The estimate for the time derivative can be verified in a completely identical manner. We now compute

$$
\begin{aligned}
& \text { Cross terms between } \phi_{0} \text { and } \sum_{\mathbf{A}} \lambda F_{\mathbf{A}} \cos \left(\frac{u_{\mathbf{A}}}{\lambda}\right) \\
& =\underbrace{-2 \sum_{\mathbf{A}} F_{\mathbf{A}} \partial_{(\mu} \phi_{0} \partial_{\nu)} u_{\mathbf{A}} \sin \left(\frac{u_{\mathbf{A}}}{\lambda}\right)}_{\mathfrak{G}_{1,1}}+\underbrace{2 \lambda \sum_{\mathbf{A}} \partial_{(\mu} \phi_{0} \partial_{\nu)} F_{\mathbf{A}} \cos \left(\frac{u_{\mathbf{A}}}{\lambda}\right)}_{\mathfrak{G}_{2,4}} .
\end{aligned}
$$




$$
\begin{aligned}
& \text { The term } \partial_{\mu}\left(\sum_{\mathbf{A}} \lambda F_{\mathbf{A}} \cos \left(\frac{u_{\mathbf{A}}}{\lambda}\right)\right) \partial_{\nu}\left(\sum_{\mathbf{B}} \lambda F_{\mathbf{B}} \cos \left(\frac{u_{\mathbf{B}}}{\lambda}\right)\right) \\
= & \sum_{\mathbf{A}, \mathbf{B}} F_{\mathbf{A}} F_{\mathbf{B}} \partial_{\mu} u_{\mathbf{A}} \partial_{\nu} u_{\mathbf{B}} \sin \left(\frac{u_{\mathbf{A}}}{\lambda}\right) \sin \left(\frac{u_{\mathbf{B}}}{\lambda}\right)-2 \lambda \sum_{\mathbf{A}, \mathbf{B}} \partial_{(\mu} F_{\mathbf{A}} \partial_{\nu)} u_{\mathbf{B}} F_{\mathbf{B}} \cos \left(\frac{u_{\mathbf{A}}}{\lambda}\right) \sin \left(\frac{u_{\mathbf{B}}}{\lambda}\right)+\ldots \\
= & \underbrace{\frac{1}{2} \sum_{\mathbf{A}} F_{\mathbf{A}}^{2}\left(\partial_{\mu} u_{\mathbf{A}}\right)\left(\partial_{\nu} u_{\mathbf{A}}\right)}_{\Theta^{(\text {main })}}+\underbrace{\frac{1}{2} \sum_{\mathbf{A}} F_{\mathbf{A}}^{2} \partial_{\mu} u_{\mathbf{A}} \partial_{\nu} u_{\mathbf{A}} \cos \left(\frac{2 u_{\mathbf{A}}}{\lambda}\right)}_{\mathfrak{G}_{1,3}} \\
& +\underbrace{\frac{1}{2} \sum_{ \pm} \sum_{\mathbf{A}} \sum_{\mathbf{B} \neq \mathbf{A}}(\mp 1) \cdot F_{\mathbf{A}} F_{\mathbf{B}} \partial_{\mu} u_{\mathbf{A}} \partial_{\nu} u_{\mathbf{B}} \cos \left(\frac{u_{\mathbf{A}} \pm u_{\mathbf{B}}}{\lambda}\right)}_{\mathfrak{G}_{2,1}} \\
& \underbrace{\lambda \sum_{ \pm} \sum_{\mathbf{A}} \sum_{\mathbf{B} \neq \mathbf{A}}( \pm 1) \partial_{(\mu} F_{\mathbf{A}} \partial_{\nu)} u_{\mathbf{B}} F_{\mathbf{B}} \sin \left(\frac{u_{\mathbf{A}} \pm u_{\mathbf{B}}}{\lambda}\right)}_{ \pm}-\lambda \underbrace{\sum_{\underbrace{}_{(\mu}} \partial_{\mathbf{A}} \partial_{\nu)} u_{\mathbf{A}} F_{\mathbf{A}} \sin \left(\frac{2 u_{\mathbf{A}}}{\lambda}\right)}_{\mathbf{A}}+\ldots,
\end{aligned}
$$

where here, and below, ... denotes terms satisfying the bound (7.5). To justify that the ... terms indeed satisfy the necessary bounds, we have used here Corollary 3.5.

Next, we use Corollary 3.5, (B1) and (BA1) to obtain

Cross terms between $\phi_{0}$ and $\sum_{\mathbf{A}} \lambda^{2} \widetilde{F}_{\mathbf{A}} \sin \left(\frac{u_{\mathbf{A}}}{\lambda}\right)+\sum_{\mathbf{A}} \lambda^{2} \widetilde{F}_{\mathbf{A}}^{(2)} \cos \left(\frac{2 u_{\mathbf{A}}}{\lambda}\right)+\sum_{\mathbf{A}} \lambda^{2} \widetilde{F}_{\mathbf{A}}^{(3)} \sin \left(\frac{3 u_{\mathbf{A}}}{\lambda}\right)$

$$
=\underbrace{2 \lambda \sum_{\mathbf{A}} \partial_{(\mu} \phi_{0} \partial_{\nu)} u_{\mathbf{A}}\left(\widetilde{F}_{\mathbf{A}} \cos \left(\frac{u_{\mathbf{A}}}{\lambda}\right)-2 \widetilde{F}_{\mathbf{A}}^{(2)} \sin \left(\frac{2 u_{\mathbf{A}}}{\lambda}\right)+3 \widetilde{F}_{\mathbf{A}}^{(3)} \cos \left(\frac{3 u_{\mathbf{A}}}{\lambda}\right)\right)}_{\mathfrak{G}_{2,3}}+\ldots
$$

Using again Corollary 3.5, (B1) and (BA1), we have

$$
\begin{aligned}
& \text { Cross terms between } \sum_{\mathbf{A}} \lambda F_{\mathbf{A}} \cos \left(\frac{u_{\mathbf{A}}}{\lambda}\right) \\
& \text { and } \sum_{\mathbf{B}} \lambda^{2} \widetilde{F}_{\mathbf{B}} \sin \left(\frac{u_{\mathbf{B}}}{\lambda}\right)+\sum_{\mathbf{B}} \lambda^{2} \widetilde{F}_{\mathbf{B}}^{(2)} \cos \left(\frac{2 u_{\mathbf{B}}}{\lambda}\right)+\sum_{\mathbf{B}} \lambda^{2} \widetilde{F}_{\mathbf{B}}^{(3)} \sin \left(\frac{3 u_{\mathbf{B}}}{\lambda}\right) \\
& =\underbrace{-2 \lambda \sum_{\mathbf{A}, \mathbf{B}} \partial_{\mu} u_{\mathbf{A}} \partial_{\nu} u_{\mathbf{B}} F_{\mathbf{A}} \sin \left(\frac{u_{\mathbf{A}}}{\lambda}\right)\left(\widetilde{F}_{\mathbf{B}} \cos \left(\frac{u_{\mathbf{B}}}{\lambda}\right)-2 \widetilde{F}_{\mathbf{B}}^{(2)} \sin \left(\frac{2 u_{\mathbf{B}}}{\lambda}\right)+3 \widetilde{F}_{\mathbf{B}}^{(3)} \cos \left(\frac{3 u_{\mathbf{B}}}{\lambda}\right)\right)+\ldots}_{\mathfrak{G}_{2,5}}
\end{aligned}
$$

In (7.6), (7.7), (7.8) and (7.9), we have seen all of the $\Theta_{\mu \nu}^{(\text {main })}, \Theta_{\mu \nu}^{(1)}$ and $\Theta_{\mu \nu}^{(2)}$ terms. Therefore, it suffices to show that all remaining terms satisfy the estimate (7.5). 
For the cross terms between $\sum_{\mathbf{A}} \lambda^{2} \widetilde{F}_{\mathbf{A}} \sin \left(\frac{u_{\mathbf{A}}}{\lambda}\right), \sum_{\mathbf{A}} \lambda^{2} \widetilde{F}_{\mathbf{A}}^{(2)} \cos \left(\frac{2 u_{\mathbf{A}}}{\lambda}\right)$ and $\sum_{\mathbf{A}} \lambda^{2} \widetilde{F}_{\mathbf{A}}^{(3)} \sin \left(\frac{3 u_{\mathbf{A}}}{\lambda}\right)$, we argue as follows. For the $\widetilde{F} \cdot \widetilde{F}$ term, we have

$$
\begin{aligned}
& \sum_{k \leq 3} \lambda^{k}\|\cdot\|_{H^{k}} \\
& \leq \sum_{k \leq 3} \sum_{\mathbf{A}, \mathbf{B}} \lambda^{k+2}\left(\sum_{k_{1}+k_{2}=k} \lambda^{-k_{1}}\left\|\left(\widetilde{F}_{\mathbf{A}}\right)\left(\widetilde{F}_{\mathbf{B}}\right)\right\|_{H^{k_{2}}}+\sum_{k_{1}+k_{2}=k} \lambda^{-k_{1}+1}\left\|\widetilde{F}_{\mathbf{A}}\left(\partial \widetilde{F}_{\mathbf{B}}\right)\right\|_{H^{k_{2}}}\right. \\
&\left.+\sum_{k_{1}+k_{2}=k} \lambda^{-k_{1}+2}\left\|\left(\partial \widetilde{F}_{\mathbf{A}}\right)\left(\partial \widetilde{F}_{\mathbf{B}}\right)\right\|_{H^{k_{2}}}\right) \\
& \leq C\left(C_{0}\right) \sum_{k \leq 3} \sum_{\mathbf{A}, \mathbf{B}} \lambda^{k+2}\left(\left\|\widetilde{F}_{\mathbf{A}}\right\|_{L^{\infty}} \sum_{k_{1}+k_{2}=k}\left(\lambda^{-k_{1}}\left\|\widetilde{F}_{\mathbf{B}}\right\|_{H^{k_{2}}}+\lambda^{-k_{1}+1}\left\|\partial \widetilde{F}_{\mathbf{B}}\right\|_{H^{k_{2}}}\right)\right. \\
&\left.\quad+\left\|\partial \widetilde{F}_{\mathbf{A}}\right\|_{L^{\infty}} \sum_{k_{1}+k_{2}=k}\left(\lambda^{-k_{1}+1}\left\|\widetilde{F}_{\mathbf{B}}\right\|_{H^{k_{2}}}+\lambda^{-k_{1}+2}\left\|\partial \widetilde{F}_{\mathbf{B}}\right\|_{H^{k_{2}}}\right)\right) \\
& \leq C\left(C_{1}\right) \varepsilon \lambda^{2},
\end{aligned}
$$

as desired. Here, in deriving (7.10), we have used

- the fact that (by Corollary 3.5) every derivative of the phase function gives $\lambda^{-1}$,

- the standard product estimate in Proposition A.7.

- the bootstrap assumption (BA1) to control the $H^{k}$ norms of $\widetilde{F}_{\mathbf{A}}$ and $\partial \widetilde{F}_{\mathbf{A}}$,

- the estimates in (5.4) to control the $L^{\infty}$ norms of $\widetilde{F}_{\mathbf{A}}$ and $\partial \widetilde{F}_{\mathbf{A}}$.

Next, we note that in the estimate (7.10), if we replace one (or both) of the $\widetilde{F}_{\mathbf{A}}$ by $\widetilde{F}_{\mathbf{A}}^{(a)}$ for $a=2,3$, the exact same estimate applies. (In fact, it is slightly better since according to (B1), $\widetilde{F}_{\mathbf{A}}^{(a)}$ satisfies better bounds.) Therefore, all the cross terms between $\sum_{\mathbf{A}} \lambda^{2} \widetilde{F}_{\mathbf{A}} \sin \left(\frac{u_{\mathbf{A}}}{\lambda}\right), \sum_{\mathbf{A}} \lambda^{2} \widetilde{F}_{\mathbf{A}}^{(2)} \cos \left(\frac{2 u_{\mathbf{A}}}{\lambda}\right)$ and $\sum_{\mathbf{A}} \lambda^{2} \widetilde{F}_{\mathbf{A}}^{(3)} \sin \left(\frac{3 u_{\mathbf{A}}}{\lambda}\right)$ can be dealt with similarly.

Finally, it remains to estimate the term $2 \partial_{(\mu} \mathcal{E}_{\lambda} \partial_{\nu)} \phi_{\lambda}$. We now consider each term in the parametrix (4.18) and bound them. Below, we will often use the product estimate Proposition A.7 and also the simpler

$$
\|u v\|_{H^{k}} \leq\|u\|_{H^{k}}\|v\|_{C^{k}}
$$

as well as the Sobolev embedding (Proposition A.4, and especially the $H^{2} \subset L^{\infty}$ embedding). We will use these estimates without further comments.

Using Corollaries 3.5 and 6.23, we have

$$
\sum_{k \leq 3} \lambda^{k}\left\|\partial \mathcal{E}_{\lambda} \partial \phi_{0}\right\|_{H^{k}} \leq C\left(C_{0}\right) \sum_{k \leq 3} \lambda^{k}\left\|\partial \mathcal{E}_{\lambda}\right\|_{H^{k}}\left\|\partial \phi_{0}\right\|_{H^{5}} \leq C\left(C_{1}\right) \varepsilon^{2} \lambda^{2}
$$

Using Corollaries 3.5 and 6.23, we have

$$
\begin{aligned}
& \sum_{k \leq 3} \sum_{\mathbf{A}} \lambda^{k}\left\|\left(\partial \mathcal{E}_{\lambda}\right)\left(\partial \sum_{\mathbf{A}} \lambda F_{\mathbf{A}} \cos \left(\frac{u_{\mathbf{A}}}{\lambda}\right)\right)\right\|_{H^{k}} \\
\leq & C\left(C_{0}\right) \sum_{k \leq 3} \sum_{\mathbf{A}} \lambda^{k} \sum_{k_{1}+k_{2}=k}\left\|\partial \mathcal{E}_{\lambda}\right\|_{H^{k_{1}}}\left\|\partial\left(\lambda F_{\mathbf{A}} \cos \left(\frac{u_{\mathbf{A}}}{\lambda}\right)\right)\right\|_{C^{k_{2}}} \\
\leq & C\left(C_{1}\right) \varepsilon^{2} \sum_{k \leq 3} \lambda^{k} \sum_{k_{1}+k_{2}=k} \lambda^{2-k_{1}-k_{2}} \leq C\left(C_{1}\right) \varepsilon^{2} \lambda^{2} .
\end{aligned}
$$


Using the bootstrap assumption (BA1), the estimates (5.4), (5.6), Corollaries 3.5 and 6.23, we have

$$
\begin{aligned}
& \sum_{k \leq 3} \lambda^{k}\left\|\left(\partial \mathcal{E}_{\lambda}\right)\left(\partial \sum_{\mathbf{A}} \lambda^{2} \widetilde{F}_{\mathbf{A}} \sin \left(\frac{u_{\mathbf{A}}}{\lambda}\right)\right)\right\|_{H^{k}} \\
\leq & C\left(C_{0}\right) \sum_{k \leq 3} \sum_{\mathbf{A}} \lambda^{k}\left(\left\|\partial \mathcal{E}_{\lambda}\right\|_{H^{k}}\left(\lambda^{2}\left\|\partial \widetilde{F}_{\mathbf{A}}\right\|_{L^{\infty}}+\lambda\left\|\widetilde{F}_{\mathbf{A}}\right\|_{L^{\infty}}\right)\right. \\
& \left.+\left\|\partial \mathcal{E}_{\lambda}\right\|_{L^{\infty}}\left(\sum_{k_{1}+k_{2}=k} \lambda^{-k_{1}+2}\left\|\partial \widetilde{F}_{\mathbf{A}}\right\|_{H^{k_{2}}}+\sum_{k_{1}+k_{2}=k} \lambda^{-k_{1}+1}\left\|\widetilde{F}_{\mathbf{A}}\right\|_{H^{k_{2}}}\right)\right) \\
\leq & C\left(C_{1}\right) \sum_{k \leq 3} \lambda^{k}\left(\varepsilon^{2} \lambda^{2-k} \cdot\left(\lambda^{2} \cdot \lambda^{-1}+\lambda\right)+\varepsilon^{2} \lambda \sum_{k_{1}+k_{2}=k} \lambda^{-k_{1}+2} \lambda^{\min \left\{-k_{2}+1,0\right\}}\right. \\
& \left.+\varepsilon^{2} \lambda \sum_{k_{1}+k_{2}=k} \lambda^{-k_{1}+1} \lambda^{\min \left\{-k_{2}+2,0\right\}}\right) \\
\leq & C\left(C_{1}\right) \varepsilon^{2} \lambda^{2} .
\end{aligned}
$$

Next, we note that $\widetilde{F}_{\mathbf{A}}^{(2)}$ and $\widetilde{F}_{\mathbf{A}}^{(3)}$ both obey better bounds than $\widetilde{F}_{\mathbf{A}}$ according to (B1) and (5.5). Therefore, we can argue similarly as above to get

$$
\begin{aligned}
& \sum_{k \leq 3} \lambda^{k}\left(\left\|\left(\partial \mathcal{E}_{\lambda}\right)\left(\partial \sum_{\mathbf{A}} \lambda \widetilde{F}_{\mathbf{A}}^{(2)} \cos \left(\frac{2 u_{\mathbf{A}}}{\lambda}\right)\right)\right\|_{H^{k}}+\left\|\left(\partial \mathcal{E}_{\lambda}\right)\left(\partial \sum_{\mathbf{A}} \lambda \widetilde{F}_{\mathbf{A}}^{(3)} \sin \left(\frac{3 u_{\mathbf{A}}}{\lambda}\right)\right)\right\|_{H^{k}}\right) \\
& \leq C\left(C_{1}\right) \varepsilon^{2} \lambda^{2} .
\end{aligned}
$$

Finally, using Corollary 6.23, we obtain

$$
\sum_{k \leq 3} \lambda^{k}\left\|\left(\partial \mathcal{E}_{\lambda}\right)^{2}\right\|_{H^{k}} \leq C\left(C_{0}\right) \sum_{k \leq 3} \lambda^{k}\left\|\partial \mathcal{E}_{\lambda}\right\|_{L^{\infty}}\left\|\partial \mathcal{E}_{\lambda}\right\|_{H^{k}} \leq C\left(C_{0}\right) \sum_{k \leq 3} \lambda^{k}\left\|\partial \mathcal{E}_{\lambda}\right\|_{H^{2}}\left\|\partial \mathcal{E}_{\lambda}\right\|_{H^{k}} \leq C\left(C_{1}\right) \varepsilon^{2} \lambda^{2} .
$$

The following proposition shows that $\mathfrak{g}_{1}$ (defined in (4.37)) is chosen so that $\Delta \mathfrak{g}_{1}$ cancels with the term $\Theta^{(1)}$, at the expense of some additional $O(\lambda)$ terms.

Proposition 7.2. For $\mathfrak{g}_{1}$ as in (4.37) and $\boldsymbol{\Gamma}_{0}(\mathfrak{g})$ as in (4.28), (4.29), (4.30) and (4.31) (with metric components replaced by their background values) and $\Theta^{(1)}$ as in (7.3), we have that $\Delta \mathfrak{g}_{1}-\Gamma_{0}(\mathfrak{g})^{\mu \nu} \Theta_{\mu \nu}^{(1)}$ depends only on the background solution with

$$
\begin{aligned}
& \Delta \mathfrak{g}_{1}-\boldsymbol{\Gamma}_{0}(\mathfrak{g})^{\mu \nu} \Theta_{\mu \nu}^{(1)} \\
= & \lambda \sum_{\mathbf{A}}\left(\mathcal{G}_{1,1, \mathbf{A}}^{(\Delta)}(\mathfrak{g}) \cos \left(\frac{u_{\mathbf{A}}}{\lambda}\right)+\mathcal{G}_{1,2, \mathbf{A}}^{(\Delta)}(\mathfrak{g}) \sin \left(\frac{2 u_{\mathbf{A}}}{\lambda}\right)\right) \\
& +\lambda \sum_{ \pm} \sum_{\mathbf{A}} \sum_{\mathbf{B} \neq \mathbf{A}} \mathcal{G}_{2,1, \mathbf{A}, \mathbf{B}, \pm}^{(\Delta)}(\mathfrak{g}) \sin \left(\frac{u_{\mathbf{A}} \pm u_{\mathbf{B}}}{\lambda}\right)+\mathcal{G}_{\text {error }}^{(\Delta)}(\mathfrak{g})
\end{aligned}
$$

for some $\mathcal{G}_{1,1, \mathbf{A}}^{(\Delta)}(\mathfrak{g}), \mathcal{G}_{1,2, \mathbf{A}}^{(\Delta)}(\mathfrak{g}), \mathcal{G}_{2,1, \mathbf{A}, \mathbf{B}, \pm}^{(\Delta)}(\mathfrak{g})$ and $\mathcal{G}_{\text {error }}^{(\Delta)}(\mathfrak{g})$ such that (for any $\mathfrak{g} \in\left\{N, \gamma, \beta^{i}\right\}$ )

- $\mathcal{G}_{1,1, \mathbf{A}}^{(\Delta)}(\mathfrak{g}), \mathcal{G}_{1,2, \mathbf{A}}^{(\Delta)}(\mathfrak{g})$ and $\mathcal{G}_{2,1, \mathbf{A}, \mathbf{B}, \pm}^{(\Delta)}(\mathfrak{g})$ are all compactly supported in $B\left(0, R_{\text {supp }}\right)$ in the time interval $[0,1]$ and obey the estimates

$$
\|\cdot\|_{H^{7} \cap C^{7}}+\left\|\partial_{t}(\cdot)\right\|_{H^{6} \cap C^{6}} \leq C\left(C_{0}\right) \varepsilon^{2}
$$

- $\mathcal{G}_{\text {error }}^{(\Delta)}(\mathfrak{g})$ is also compactly supported in $B\left(0, R_{\text {supp }}\right)$ in the time interval $[0,1]$ and obeys the estimate

$$
\sum_{k \leq 3} \lambda^{k}\left\|\mathcal{G}_{\text {error }}^{(\Delta)}(\mathfrak{g})\right\|_{H^{k}}+\sum_{k \leq 2} \lambda^{k+1}\left\|\partial_{t} \mathcal{G}_{\text {error }}^{(\Delta)}(\mathfrak{g})\right\|_{H^{k}} \leq C\left(C_{0}\right) \varepsilon^{2} \lambda^{2} .
$$

Proof. Note that the definition of $\mathfrak{g}_{1}$ in (4.37) is such that if each derivative of $\Delta$ hits the oscillating factor, the resulting term cancels exactly $\Gamma_{0}^{\mu \nu}(\mathfrak{g}) \Theta_{\mu \nu}^{(1)}$.

In the case that exactly one derivative of $\Delta$ hits the oscillating factor, then the phases become $\sin \left(\frac{2 u_{A}}{\lambda}\right)$, $\cos \left(\frac{u_{\mathrm{A}}}{\lambda}\right)$ and $\sin \left(\frac{u_{\mathrm{A}} \pm u_{\mathrm{B}}}{\lambda}\right)$. This then gives rise to the three main terms in the proposition, and one checks 
using the definition of $\mathfrak{g}_{1}$ from (4.37) that $\mathcal{G}_{1,1, \mathbf{A}}^{(\Delta)}(\mathfrak{g}), \mathcal{G}_{1,2, \mathbf{A}}^{(\Delta)}(\mathfrak{g}), \mathcal{G}_{2,1, \mathbf{A}, \mathbf{B}, \pm}^{(\Delta)}(\mathfrak{g})$ all depend only on the background solution, are compactly supported in $[0,1] \times B\left(0, R_{\text {supp }}\right)$ and obey the stated bounds.

Finally, denote by $\mathcal{G}_{\text {error }}^{(\Delta)}(\mathfrak{g})$ all the terms that $\Delta$ does not hit on the oscillating factor. It is easy to check using (4.37) that it has all the desired properties.

Proposition 7.3. For $\boldsymbol{\Gamma}_{0}^{\mu \nu}(\mathfrak{g})$ as in (4.28), (4.29), (4.30) and (4.31) (with metric components replaced by their background values) and $\Theta^{(2)}$ as in (7.4), $\Gamma_{0}^{\mu \nu}(\mathfrak{g}) \Theta_{\mu \nu}^{(2)}$ can be written as

$$
\begin{aligned}
\Gamma_{0}^{\mu \nu}(\mathfrak{g}) \Theta_{\mu \nu}^{(2)}= & \sum_{\mathbf{A}} \lambda \mathcal{G}_{1,1, \mathbf{A}}^{(\Theta)}(\mathfrak{g}) \cos \left(\frac{u_{\mathbf{A}}}{\lambda}\right)+\sum_{\mathbf{A}} \lambda \mathcal{G}_{1,2, \mathbf{A}}^{(\Theta)}(\mathfrak{g}) \sin \left(\frac{2 u_{\mathbf{A}}}{\lambda}\right) \\
& +\sum_{\mathbf{A}} \lambda \mathcal{G}_{1,3, \mathbf{A}}^{(\Theta)}(\mathfrak{g}) \cos \left(\frac{3 u_{\mathbf{A}}}{\lambda}\right)+\sum_{ \pm} \sum_{\mathbf{A}} \sum_{\mathbf{B} \neq \mathbf{A}} \lambda \mathcal{G}_{2,1, \mathbf{A}, \mathbf{B}, \pm}^{(\Theta)}(\mathfrak{g}) \sin \left(\frac{u_{\mathbf{A}} \pm u_{\mathbf{B}}}{\lambda}\right) \\
& +\sum_{ \pm} \sum_{\mathbf{A}} \sum_{\mathbf{B} \neq \mathbf{A}} \lambda \mathcal{G}_{2,2, \mathbf{A}, \mathbf{B}, \pm}^{(\Theta)}(\mathfrak{g}) \cos \left(\frac{u_{\mathbf{A}} \pm 2 u_{\mathbf{B}}}{\lambda}\right) \\
& +\sum_{ \pm} \sum_{\mathbf{A}, \mathbf{B}} \lambda \mathcal{G}_{2,3, \mathbf{A}, \mathbf{B}, \pm}^{(\Theta)}(\mathfrak{g}) \sin \left(\frac{u_{\mathbf{A}} \pm 3 u_{\mathbf{B}}}{\lambda}\right),
\end{aligned}
$$

for some $\mathcal{G}_{1,1, \mathbf{A}}^{(\Theta)}(\mathfrak{g}), \mathcal{G}_{1,2, \mathbf{A}}^{(\Theta)}(\mathfrak{g}), \mathcal{G}_{1,3, \mathbf{A}}^{(\Theta)}(\mathfrak{g}), \mathcal{G}_{2,1, \mathbf{A}, \mathbf{B}, \pm}^{(\Theta)}(\mathfrak{g}), \mathcal{G}_{2,2, \mathbf{A}, \mathbf{B}, \pm}^{(\Theta)}(\mathfrak{g}), \mathcal{G}_{2,3, \mathbf{A}, \mathbf{B}, \pm}^{(\Theta)}(\mathfrak{g})$ where each of them is compactly supported in $B\left(0, R_{\text {supp }}\right)$ and satisfies the estimate

$$
\sum_{k \leq 3} \lambda^{k}\|\cdot\|_{H^{2+k}}+\lambda^{k}\left\|\partial_{t}(\cdot)\right\|_{H^{k+1}} \leq C\left(C_{1}\right) \varepsilon^{2} .
$$

Proof. The factor $\Gamma_{0}^{\mu \nu}(\mathfrak{g})$, which depends only on the background metric, clearly obeys much better bound than we need according to Corollary 3.5. It therefore suffices to control $\Theta_{\mu \nu}^{(2)}$ from (7.4). According to (7.4), $\Theta_{\mu \nu}^{(2)}$ indeed can be expanded into a sum of terms, each with a high frequency oscillating factor as in the statement of the proposition. We thus define the $\mathcal{G}^{(\Theta)}$ terms accordingly. Most of these $\mathcal{G}^{(\Theta)}$ terms depend only on the background, and they obey the desired bounds according to Corollary [3.5) and (B1). The only $\mathcal{G}^{(\Theta)}$ terms which do not depend only on the background are of the form $F_{\mathbf{A}} \widetilde{F}_{\mathbf{B}}$ or $\partial \phi_{0} \widetilde{F}_{\mathbf{A}}$ : to show that they obey the desired estimates, we use (BA1), in addition to Corollary 3.5 and (B1). We remark that it is exactly the application of (BA1) that limits the regularity of $\mathcal{G}^{(\Theta)}$.

\section{Proposition 7.4.}

$$
\sum_{k \leq 3} \lambda^{k}\left\|\left(\boldsymbol{\Gamma}^{\mu \nu}(\mathfrak{g})-\boldsymbol{\Gamma}_{0}^{\mu \nu}(\mathfrak{g})\right) \partial_{\mu} \phi \partial_{\nu} \phi\right\|_{H^{k}}+\sum_{k \leq 2} \lambda^{k+1}\left\|\partial_{t}\left(\left(\boldsymbol{\Gamma}^{\mu \nu}(\mathfrak{g})-\boldsymbol{\Gamma}_{0}^{\mu \nu}(\mathfrak{g})\right) \partial_{\mu} \phi \partial_{\nu} \phi\right)\right\|_{H^{k}} \leq C\left(C_{1}\right) \varepsilon^{2} \lambda^{2} .
$$

Proof. Using the compact support of $\phi$, Proposition $\$$.7 gives

$$
\begin{aligned}
& \sum_{k \leq 3} \lambda^{k}\left\|\left(\boldsymbol{\Gamma}^{\mu \nu}(\mathfrak{g})-\Gamma_{0}^{\mu \nu}(\mathfrak{g})\right) \partial_{\mu} \phi \partial_{\nu} \phi\right\|_{H^{k}} \\
\leq & C\left(C_{0}\right)\left(\|\partial \phi\|_{L^{\infty}}^{2} \sum_{k \leq 3} \lambda^{k}\left\|\boldsymbol{\Gamma}^{\mu \nu}(\mathfrak{g})-\boldsymbol{\Gamma}_{0}^{\mu \nu}(\mathfrak{g})\right\|_{H^{k}\left(B\left(0, R_{\text {supp }}+1\right)\right)}\right. \\
& \left.+\left\|\boldsymbol{\Gamma}^{\mu \nu}(\mathfrak{g})-\boldsymbol{\Gamma}_{0}^{\mu \nu}(\mathfrak{g})\right\|_{L^{\infty}\left(B\left(0, R_{\text {supp }}+1\right)\right)}\|\partial \phi\|_{L^{\infty}} \sum_{k \leq 3} \lambda^{k}\|\partial \phi\|_{H^{k}}\right) \\
\leq & C\left(C_{1}\right)\left(\varepsilon^{2} \cdot \varepsilon \lambda^{2}+\varepsilon \lambda^{2} \cdot \varepsilon \cdot \varepsilon\right) \leq C\left(C_{1}\right) \varepsilon^{2} \lambda^{2},
\end{aligned}
$$

where in the last step we have used the estimates in (B1), (B2), (BA1), (BA2), (BA3), (BA4), (5.7), (5.8) and Corollary 6.23,

7.2. Definition of $\mathfrak{g}_{2}$. Our goal in this subsection is to show that $\Delta\left(\mathfrak{g}-\mathfrak{g}_{0}-\mathfrak{g}_{1}-\mathfrak{g}_{2}\right)$ is appropriately small. This of course involve a suitable choice of $\mathfrak{g}_{2}$. Recall from (4.38) that we have given an expression for $\mathfrak{g}_{2}$, but the functions $\mathcal{G}_{1,1, \mathbf{A}}(\mathfrak{g}), \mathcal{G}_{1,2, \mathbf{A}}(\mathfrak{g}), \mathcal{G}_{1,3, \mathbf{A}}(\mathfrak{g}), \mathcal{G}_{2,1, \mathbf{A}, \mathbf{B}, \pm}(\mathfrak{g}), \mathcal{G}_{2,2, \mathbf{A}, \mathbf{B}, \pm}(\mathfrak{g})$ and $\mathcal{G}_{2,3, \mathbf{A}, \mathbf{B}, \pm}(\mathfrak{g})$ are 
yet to be defined. They will be defined in this subsection. Together with the calculations from the previous subsection, we then obtain a good estimate for $\Delta\left(\mathfrak{g}-\mathfrak{g}_{0}-\mathfrak{g}_{1}-\mathfrak{g}_{2}\right)$.

Before we define $\mathfrak{g}_{2}$, we need another piece of notation. Let (compare (4.32), (4.33) and (4.34))

$$
\begin{aligned}
& \Upsilon_{1}(\gamma):=-\frac{e^{2 \gamma_{0}}}{4 N_{0}^{2}} \delta^{i j} \delta^{k \ell}\left(L \beta_{1}\right)_{i k}\left(L \beta_{0}\right)_{j \ell}, \\
& \Upsilon_{1}(N):=\frac{e^{2 \gamma_{0}}}{2 N_{0}} \delta^{i j} \delta^{k \ell}\left(L \beta_{1}\right)_{i k}\left(L \beta_{0}\right)_{j \ell}, \\
& \Upsilon_{1}\left(\beta^{i}\right):=\delta^{j k} \delta^{i \ell}\left(\left(\partial_{k} \log N_{0}\right)\left(L \beta_{1}\right)_{j \ell}+\left(\partial_{k} \log N_{1}\right)\left(L \beta_{0}\right)_{j \ell}-2\left(\partial_{k} \gamma_{0}\right)\left(L \beta_{1}\right)_{j \ell}-2\left(\partial_{k} \gamma_{1}\right)\left(L \beta_{0}\right)_{j \ell}\right) .
\end{aligned}
$$

The terms defined above are meant to be the "main term" in $\Upsilon-\Upsilon_{0}$. We will show that it also can be decomposed into the "right type" of high frequency terms so that we can define $\mathfrak{g}_{2}$ in the parametrix appropriately to "remove this main term" in $\Delta\left(\mathfrak{g}-\mathfrak{g}_{0}-\mathfrak{g}_{1}\right)$. This is made precise in the next two propositions.

Proposition 7.5. $\Upsilon_{1}(\mathfrak{g})$ can be written as

$$
\begin{aligned}
\Upsilon_{1}(\mathfrak{g})= & \lambda \sum_{\mathbf{A}}\left(\mathcal{G}_{1,1, \mathbf{A}}^{(\Upsilon)}(\mathfrak{g}) \cos \left(\frac{u_{\mathbf{A}}}{\lambda}\right)+\mathcal{G}_{1,2, \mathbf{A}}^{(\Upsilon)}(\mathfrak{g}) \sin \left(\frac{2 u_{\mathbf{A}}}{\lambda}\right)\right) \\
& +\lambda \sum_{ \pm} \sum_{\mathbf{A}} \sum_{\mathbf{B} \neq \mathbf{A}} \mathcal{G}_{2,1, \mathbf{A}, \mathbf{B}, \pm}^{(\Upsilon)}(\mathfrak{g}) \sin \left(\frac{u_{\mathbf{A}} \pm u_{\mathbf{B}}}{\lambda}\right)+\mathcal{G}_{\text {error }}^{(\Upsilon)}(\mathfrak{g}),
\end{aligned}
$$

for some $\mathcal{G}_{1,1, \mathbf{A}}^{(\Upsilon)}(\mathfrak{g}), \mathcal{G}_{1,2, \mathbf{A}}^{(\Upsilon)}(\mathfrak{g})$ and $\mathcal{G}_{2,1, \mathbf{A}, \mathbf{B}, \pm}^{(\mathfrak{)})}\left(\mathfrak{g}\right.$ wich are all compactly supported in $B\left(0, R_{\text {supp }}\right)$ in the time interval $[0,1]$ and obey the estimates

$$
\|\cdot\|_{H^{7} \cap C^{7}}+\left\|\partial_{t}(\cdot)\right\|_{H^{6} \cap C^{6}} \leq C\left(C_{0}\right) \varepsilon^{2},
$$

and some $\mathcal{G}_{\text {error }}^{(\Upsilon)}(\mathfrak{g})$ also compactly supported in $B\left(0, R_{\text {supp }}\right)$ in the time interval $[0,1]$ and which obeys the estimate

$$
\sum_{k \leq 3} \lambda^{k}\left\|\mathcal{G}_{\text {error }}^{(\Upsilon)}(\mathfrak{g})\right\|_{H^{k}}+\sum_{k \leq 2} \lambda^{k+1}\left\|\partial_{t} \mathcal{G}_{\text {error }}^{(\Upsilon)}(\mathfrak{g})\right\|_{H^{k}} \leq C\left(C_{0}\right) \varepsilon^{2} \lambda^{2}
$$

Proof. This follows immediately from the definition of $\mathfrak{g}_{1}$ in (4.37), the definition of $\Upsilon_{1}$ in (7.11), (7.12) and (7.13) and the estimates for the background solution in Corollary 3.5.

\section{Proposition 7.6.}

$$
\sum_{k \leq 3} \lambda^{k}\left\|\Upsilon(\mathfrak{g})-\Upsilon_{0}(\mathfrak{g})-\Upsilon_{1}(\mathfrak{g})\right\|_{H_{\delta+2}^{k}}+\sum_{k \leq 2} \lambda^{k+1}\left\|\partial_{t}\left(\Upsilon(\mathfrak{g})-\Upsilon_{0}(\mathfrak{g})-\Upsilon_{1}(\mathfrak{g})\right)\right\|_{H_{\delta+2}^{k}} \leq C\left(C_{1}\right) \varepsilon^{2} \lambda^{2} .
$$

Proof. Structure of the terms. First, $\Upsilon(\mathfrak{g}), \Upsilon_{0}(\mathfrak{g})$ and $\Upsilon_{1}(\mathfrak{g})$ are defined such that $\Upsilon(\mathfrak{g})-\Upsilon_{0}(\mathfrak{g})-\Upsilon_{1}(\mathfrak{g})$ can be written as a sum of terms of the following types:

(I) a term with a factor of $\mathfrak{g}-\mathfrak{g}_{0}$ (without derivative) multiplied by factors depending on $\nabla \mathfrak{g}$,

(II) a term with a quadratic factor $\left(\nabla \mathfrak{g}_{1}+\nabla \mathfrak{g}_{2}+\nabla \mathfrak{g}_{3}\right) \cdot\left(\nabla \mathfrak{g}_{1}+\nabla \mathfrak{g}_{2}+\nabla \mathfrak{g}_{3}\right)$, multiplied by factors depending on the background metric $\mathfrak{g}_{0}$,

(III) a term with a factor of $\nabla \mathfrak{g}_{2}$ or a factor of $\nabla \mathfrak{g}_{3}$, multiplied by factors depending on $\mathfrak{g}_{0}$ and $\nabla \mathfrak{g}_{0}$.

The key point here is that there are no terms which are linear in $\nabla \mathfrak{g}_{1}$ (and, say, multiplied by background quantities). Such terms would give estimates which are $O_{\varepsilon}(\lambda)$ instead of $O_{\varepsilon}\left(\lambda^{2}\right)$. To illustrate this structure, consider $\mathfrak{g}=\gamma$ (the other cases are similar):

$$
\begin{aligned}
& \Upsilon(\gamma)-\Upsilon_{0}(\gamma)-\Upsilon_{1}(\gamma) \\
= & \left(-\frac{e^{2 \gamma}}{8 N^{2}}+\frac{e^{2 \gamma_{0}}}{8 N_{0}^{2}}\right)|L \beta|^{2}+\frac{e^{2 \gamma_{0}}}{8 N_{0}^{2}}\left(\delta^{i j} \delta^{k \ell}(L \beta)_{i k}(L \beta)_{j \ell}-\delta^{i j} \delta^{k \ell}\left(L \beta_{0}\right)_{i k}\left(L \beta_{0}\right)_{j \ell}-2 \delta^{i j} \delta^{k \ell}\left(L \beta_{1}\right)_{i k}\left(L \beta_{0}\right)_{j \ell}\right) \\
= & \underbrace{\left(-\frac{e^{2 \gamma}}{8 N^{2}}+\frac{e^{2 \gamma_{0}}}{8 N_{0}^{2}}\right)|L \beta|^{2}}_{=: I}+\frac{e^{2 \gamma_{0}}}{8 N_{0}^{2}}(\underbrace{\left|L \beta-L \beta_{0}\right|^{2}}_{=: I I}+\underbrace{2 \delta^{i j} \delta^{k \ell}\left(L \beta-L \beta_{0}-L \beta_{1}\right)_{i k}\left(L \beta_{0}\right)_{j \ell}}_{=: I I I}) .
\end{aligned}
$$

Note that the terms $I, I I$ and $I I I$ exactly satisfy the conditions $I, I I$ and $I I I$ above.

Decay at infinity. We consider first the issue of decay at infinity. Let us first consider the product of the spatial derivative of $\mathfrak{g}$. This concerns only products of the form $\nabla \mathfrak{g}_{0} \nabla \mathfrak{g}_{3}$ and $\nabla \mathfrak{g}_{3} \nabla \mathfrak{g}_{3}$, since the other terms contain at least a factor which is compactly supported. Now, $\mathfrak{g}_{0}=\chi(|x|) \log (|x|)\left(\mathfrak{g}_{0}\right)_{\text {asymp }}+\widetilde{\mathfrak{g}}_{0}$ and 
$\mathfrak{g}_{3}=\chi(|x|) \log (|x|)\left(\mathfrak{g}_{3}\right)_{\text {asymp }}+\widetilde{\mathfrak{g}}_{3}$, where $\left(\mathfrak{g}_{0}\right)_{\text {asymp }}$ and $\left(\mathfrak{g}_{3}\right)_{\text {asymp }}$ are independent of $x$ (but potentially $t$-dependent) and the terms $\widetilde{\mathfrak{g}}_{0}$ and $\widetilde{\mathfrak{g}}_{3}$ decay better (cf. Corollary 3.5 and $(\underline{\mathrm{BA} 4})$ ). There are three types of products:

- The product $\nabla \widetilde{\mathfrak{g}}_{3} \nabla \widetilde{\mathfrak{g}}_{3}$ or $\nabla \widetilde{\mathfrak{g}}_{0} \nabla \widetilde{\mathfrak{g}}_{3}$ has more decay than necessary to be in $H_{\delta+2}^{k}$ (see Proposition A.6).

- The product of the form $\nabla\left(\chi(|x|) \log (|x|)\left(\mathfrak{g}_{3}\right)_{\text {asymp }}\right) \nabla\left(\chi(|x|) \log (|x|)\left(\mathfrak{g}_{3}\right)_{\text {asymp }}\right)$ (or similarly the product $\left.\nabla\left(\chi(|x|) \log (|x|)\left(\mathfrak{g}_{0}\right)_{\text {asymp }}\right) \nabla\left(\chi(|x|) \log (|x|)\left(\mathfrak{g}_{3}\right)_{\text {asymp }}\right)\right)$ would not have enough decay, but such terms are absent, since all the terms in (4.32), (4.33), (4.34), (7.11), (7.12), (77.13) contain a factor of the form $\nabla \beta_{3}$ and $\left(\beta_{3}\right)_{\text {asymp }}=0$ by (5.2).

- It remains only to consider the "cross terms"

$$
\partial_{k}\left(\left(N_{3}\right)_{\text {asymp }} \chi(|x|) \log (|x|)\right)\left(L \beta_{3}\right)_{i \ell} \quad \partial_{k}\left(\left(\gamma_{3}\right)_{\text {asymp }} \chi(|x|) \log (|x|)\right)\left(L \beta_{3}\right)_{i \ell} .
$$

Note that the factors $\partial_{k}\left(\left(N_{3}\right)_{\text {asymp }} \chi(|x|) \log (|x|)\right)$ and $\partial_{k}\left(\left(\gamma_{3}\right)_{\text {asymp }} \chi(|x|) \log (|x|)\right)$ are $O\left(\frac{1}{|x|}\right)$ as $|x|$ tend to infinity, so the product with $L \beta$ have the right decay i.e., belongs to $H_{\delta+2}^{k}$ (see Proposition A.3).

Finally, let us note that the terms on the RHS of (4.32), (4.33), (4.34), (7.11), (7.12), (7.13) are not just products of derivatives of $\mathfrak{g}$, but has factors of $e^{2 \gamma}$ or $\frac{1}{N}$. Nevertheless, by (5.1), (5.2), $\gamma_{a s y m p} \leq 0, N_{c}=1$, $N_{\text {asymp }} \geq 1$, hence all these factors are favorable from the point of view of the weights. We will therefore suppress the discussion of them.

Proving the estimates. We now prove the estimates taking into account the discussions above. For clarity of the exposition, we will only discuss the $L_{\delta+2}^{2}$ estimates for terms of type $I, I I$ and $I I I$ discussed above. The higher derivative estimates are similar: we note that

- All the norms we use are such that every additional spatial derivative "costs" at most an extra power of $\lambda^{-1}$.

- We only use up to $H^{2}$ and $C^{1}$ norms for $\mathfrak{g}_{2}$ and $H_{\delta}^{2}$ and $C_{\delta+1}^{1}$ norms $\widetilde{\mathfrak{g}}_{3}$. Hence, according to (BA3), (BA4), (5.7) and (5.8), we still have sufficient regularity to control these terms upon taking up to 3 spatial derivatives.

- When taking higher derivatives, there may be more nonlinear terms when the derivatives hit on $e^{2 \gamma}$ or $\frac{1}{N}$. Nevertheless, these terms are all easy to handle, since $\nabla \mathfrak{g}$ is bounded in $L^{\infty}$ (and the $e^{2 \gamma}$ or $\frac{1}{N}$ terms are favorable in terms of weights).

- The above considerations also apply for up to one $\partial_{t}$ derivative, again because the additional $\partial_{t}$ derivative "costs" at most an extra power of $\lambda^{-1}$.

For terms with the structure as in $I$, we use Corollary 3.5, (B2), (BA3), (BA4), (5.7), (5.8), Lemma A.1 and Proposition A.4 to obtain

$$
\begin{aligned}
\|I\|_{L_{\delta+2}^{2}} & \lesssim\left(\left\|\mathfrak{g}_{1}\right\|_{C_{\delta+1}^{0}}+\left\|\mathfrak{g}_{2}\right\|_{C_{\delta+1}^{0}}+\left|\left(\mathfrak{g}_{3}\right)_{\text {asymp }}\right|+\left\|\widetilde{\mathfrak{g}}_{3}\right\|_{C_{\delta+1}^{0}}\right)\left(\|\nabla \widetilde{\mathfrak{g}}\|_{L_{\delta+\frac{3}{2}}^{4}}\|\nabla \widetilde{\mathfrak{g}}\|_{L_{\delta+\frac{3}{2}}^{4}}+\left|\mathfrak{g}_{\text {asymp }}\right|\|\nabla \widetilde{\mathfrak{g}}\|_{L_{\delta+1}^{2}}\right) \\
& \lesssim\left(\left\|\mathfrak{g}_{1}\right\|_{C_{\delta+1}^{0}}+\left\|\mathfrak{g}_{2}\right\|_{C_{\delta+1}^{0}}+\left|\left(\mathfrak{g}_{3}\right)_{\text {asymp }}\right|+\left\|\widetilde{\mathfrak{g}}_{3}\right\|_{C_{\delta+1}^{0}}\right)\left(\|\widetilde{\mathfrak{g}}\|_{H_{\delta}^{2}}\|\widetilde{\mathfrak{g}}\|_{H_{\delta}^{2}}+\mid \mathfrak{g}_{\text {asymp }}\|\widetilde{\mathfrak{g}}\|_{H_{\delta}^{1}}\right) \lesssim C\left(C_{1}\right) \varepsilon \lambda^{2} .
\end{aligned}
$$

For terms with the structure as in $I I$, we use (B2), (BA3), (BA4), Lemma A.1 and Proposition A.4 to obtain 33

$$
\begin{aligned}
&\|I I\|_{L_{\delta+2}^{2}} \lesssim\left(\left\|\nabla \mathfrak{g}_{1}\right\|_{L_{\delta+\frac{3}{2}}^{4}}+\left\|\nabla \mathfrak{g}_{2}\right\|_{L_{\delta+\frac{3}{2}}^{4}}+\left|\left(\mathfrak{g}_{3}\right)_{\text {asymp }}\right|+\left\|\nabla \widetilde{\mathfrak{g}}_{3}\right\|_{L_{\delta+\frac{3}{2}}^{4}}\right) \\
& \times\left(\left\|\nabla \mathfrak{g}_{1}\right\|_{L_{\delta+\frac{5}{2}}^{4}}+\left\|\nabla \mathfrak{g}_{2}\right\|_{L_{\delta+\frac{5}{2}}^{4}}+\left\|\nabla \widetilde{\mathfrak{g}}_{3}\right\|_{L_{\delta+1}^{2}}+\left\|\nabla \widetilde{\mathfrak{g}}_{3}\right\|_{L_{\delta+\frac{3}{2}}^{4}}\right) \\
& \lesssim\left(\left\|\nabla \mathfrak{g}_{1}\right\|_{L_{\delta+\frac{3}{2}}^{4}}+\left\|\mathfrak{g}_{2}\right\|_{H_{\delta}^{2}}+\left|\left(\mathfrak{g}_{3}\right)_{\text {asymp }}\right|+\left\|\widetilde{\mathfrak{g}}_{3}\right\|_{H_{\delta}^{2}}\right)\left(\left\|\nabla \mathfrak{g}_{1}\right\|_{L_{\delta+\frac{5}{2}}^{4}}+\left\|\mathfrak{g}_{2}\right\|_{H_{\delta+1}^{2}}+\left\|\widetilde{\mathfrak{g}}_{3}\right\|_{H_{\delta}^{2}}\right) \\
& \lesssim C\left(C_{1}\right) \varepsilon^{2} \lambda^{2},
\end{aligned}
$$

where we have used

$$
\left\|\nabla \mathfrak{g}_{1}\right\|_{L_{\delta+\frac{3}{2}}^{4}}+\left\|\nabla \mathfrak{g}_{1}\right\|_{L_{\delta+\frac{5}{2}}^{4}} \leq C\left(C_{0}\right) \varepsilon \lambda,
$$

which is a direct consequence of (B2), Hölder's inequality and the support properties of $\mathfrak{g}_{1}$.

\footnotetext{
${ }^{33}$ We also use the fact that $\mathfrak{g}_{1}$ and $\mathfrak{g}_{2}$ are compactly supported and we can put in arbitrary weights in the estimates.
} 
For terms with the structure as in $I I I$, we use Corollary 3.5, (BA3), (BA4), Lemma A.1 and Proposition A.4 to obtain

$$
\begin{aligned}
\|I I I\|_{L_{\delta+2}^{2}} & \lesssim\left(\left\|\nabla \mathfrak{g}_{2}\right\|_{L_{\delta+1}^{2}}+\left|\left(\mathfrak{g}_{3}\right)_{\text {asymp }}\right|+\left\|\nabla \widetilde{\mathfrak{g}}_{3}\right\|_{L_{\delta+1}^{2}}\right)\left\|\nabla \widetilde{\mathfrak{g}}_{0}\right\|_{C_{\delta+2}^{0}}+\left\|\nabla \widetilde{\mathfrak{g}}_{3}\right\|_{L_{\delta+1}^{2}}\left|\left(\mathfrak{g}_{0}\right)_{\text {asymp }}\right| \\
& \lesssim\left(\left\|\mathfrak{g}_{2}\right\|_{H_{\delta}^{1}}+\left|\left(\mathfrak{g}_{3}\right)_{\text {asymp }}\right|+\left\|\widetilde{\mathfrak{g}}_{3}\right\|_{H_{\delta}^{1}}\right)\left\|\widetilde{\mathfrak{g}}_{0}\right\|_{H_{\delta}^{3}}+\left\|\widetilde{\mathfrak{g}}_{3}\right\|_{H_{\delta}^{1}}\left|\left(\mathfrak{g}_{0}\right)_{\text {asymp }}\right| \lesssim C\left(C_{1}\right) \varepsilon^{2} \lambda^{2} .
\end{aligned}
$$

These estimates, and their higher derivative analogues, imply the conclusion of the proposition.

We now define $\mathfrak{g}_{2}$. Recall that in (4.38), we have defined $\mathfrak{g}_{2}$ modulo some functions on the RHS that we have not defined. Here, we define them using the decompositions in Propositions 7.2, 7.3 and 7.5

Definition 7.7. We define

$$
\begin{aligned}
\mathcal{G}_{1,1, \mathbf{A}}(\mathfrak{g}) & :=\mathcal{G}_{1,1, \mathbf{A}}^{(\Delta)}(\mathfrak{g})+\mathcal{G}_{1,1, \mathbf{A}}^{(\Theta)}(\mathfrak{g})+\mathcal{G}_{1,1, \mathbf{A}}^{(\Upsilon)}(\mathfrak{g}), \\
\mathcal{G}_{1,2, \mathbf{A}}(\mathfrak{g}) & :=\mathcal{G}_{1,2, \mathbf{A}}^{(\Delta)}(\mathfrak{g})+\mathcal{G}_{1,2, \mathbf{A}}^{(\Theta)}(\mathfrak{g})+\mathcal{G}_{1,2, \mathbf{A}}^{(\Upsilon)}(\mathfrak{g}), \\
\mathcal{G}_{1,3, \mathbf{A}}(\mathfrak{g}) & :=\mathcal{G}_{1,3, \mathbf{A}}^{(\Theta)}(\mathfrak{g}), \\
\mathcal{G}_{2,1, \mathbf{A}, \mathbf{B}, \pm}(\mathfrak{g}) & :=\mathcal{G}_{2,1, \mathbf{A}, \mathbf{B}, \pm}^{(\Delta)}(\mathfrak{g})+\mathcal{G}_{2,1, \mathbf{A}, \mathbf{B}, \pm}^{(\Theta)}(\mathfrak{g})+\mathcal{G}_{2,1, \mathbf{A}, \mathbf{B}, \pm}^{(\Upsilon)}(\mathfrak{g}), \\
\mathcal{G}_{2,2, \mathbf{A}, \mathbf{B}, \pm}(\mathfrak{g}) & :=\mathcal{G}_{2,2, \mathbf{A}, \mathbf{B}, \pm}^{(\Theta)}(\mathfrak{g}), \\
\mathcal{G}_{2,3, \mathbf{A}, \mathbf{B}, \pm}(\mathfrak{g}) & :=\mathcal{G}_{2,3, \mathbf{A}, \mathbf{B}, \pm}^{(\Theta)}(\mathfrak{g}),
\end{aligned}
$$

where the terms on the RHS are as in Propositions 7.2.7.3 and 7.5.

The choice of $\mathfrak{g}_{2}$ gives the following:

\section{Proposition 7.8.}

$$
\sum_{k \leq 3} \lambda^{k}\left\|\Delta \mathfrak{g}_{2}-\Gamma_{0}^{\mu \nu}(\mathfrak{g}) \Theta_{\mu \nu}^{(2)}-\Upsilon_{1}(\mathfrak{g})+\Delta \mathfrak{g}_{1}-\Gamma_{0}^{\mu \nu}(\mathfrak{g}) \Theta_{\mu \nu}^{(1)}\right\|_{H^{k}} \leq C\left(C_{1}\right) \varepsilon^{2} \lambda^{2},
$$

and

$$
\sum_{k \leq 2} \lambda^{k+1}\left\|\partial_{t}\left(\Delta \mathfrak{g}_{2}-\Gamma_{0}^{\mu \nu}(\mathfrak{g}) \Theta_{\mu \nu}^{(2)}-\Upsilon_{1}(\mathfrak{g})+\Delta \mathfrak{g}_{1}-\Gamma_{0}^{\mu \nu}(\mathfrak{g}) \Theta_{\mu \nu}^{(1)}\right)\right\|_{H^{k}} \leq C\left(C_{1}\right) \varepsilon^{2} \lambda^{2} .
$$

Proof. We will only prove the first estimate as the second estimate is similar. By (4.38), Definition 7.7 , Propositions 7.2, 7.3, 7.5, we note that $\mathfrak{g}_{2}$ is defined so that when one expands $\Delta \mathfrak{g}_{2}$, and when both derivatives hit on the highly oscillatory phase, the terms cancel with the main terms in Proposition 7.2 (i.e., the terms except for $\left.\mathcal{G}_{\text {error }}^{(\Delta)}(\mathfrak{g})\right)$, the term $\boldsymbol{\Gamma}_{0}^{\mu \nu}(\mathfrak{g}) \Theta_{\mu \nu}^{(2)}$ and the main terms in $\Upsilon_{1}(\mathfrak{g})$ (i.e., the terms except for $\left.\mathcal{G}_{\text {error }}^{(\Upsilon)}(\mathfrak{g})\right)$. Therefore, to estimate $\Delta \mathfrak{g}_{2}-\Gamma_{0}^{\mu \nu}(\mathfrak{g}) \Theta_{\mu \nu}^{(2)}-\Upsilon_{1}(\mathfrak{g})+\Delta \mathfrak{g}_{1}-\Gamma_{0}^{\mu \nu}(\mathfrak{g}) \Theta_{\mu \nu}^{(2)}$, it suffices to control the term $\mathcal{G}_{\text {error }}^{(\Delta)}(\mathfrak{g})$ from Proposition 7.2 the term $\mathcal{G}_{\text {error }}^{(\Upsilon)}(\mathfrak{g})$ in $\Upsilon_{1}(\mathfrak{g})$, and the second derivatives of $\mathfrak{g}_{2}$ where at least one derivative does not hit on the oscillating phase. Denoting by $\mathcal{G}$ any of $\mathcal{G}^{(\Delta)}, \mathcal{G}^{(\Theta)}$ or $\mathcal{G}^{(\Upsilon)}$, we have

$$
\begin{aligned}
& \sum_{k \leq 3} \lambda^{k}\left\|\Delta \mathfrak{g}_{2}-\Gamma_{0}^{\mu \nu}(\mathfrak{g}) \Theta_{\mu \nu}^{(2)}-\Upsilon_{1}(\mathfrak{g})+\Delta \mathfrak{g}_{1}-\Gamma_{0}^{\mu \nu}(\mathfrak{g}) \Theta_{\mu \nu}^{(2)}\right\|_{H^{k}} \\
\lesssim & \sum_{k \leq 3} \lambda^{k}\left(\left\|\mathcal{G}_{\text {error }}^{(\Delta)}(\mathfrak{g})\right\|_{H^{k}}+\left\|\mathcal{G}_{\text {error }}^{(\Upsilon)}(\mathfrak{g})\right\|_{H^{k}}\right)+\sum_{k \leq 3} \lambda^{k} \sum_{\ell \leq k}\left(\lambda^{3+\ell-k}\|\mathcal{G}\|_{H^{2+\ell}}+\lambda^{2+\ell-k}\|\mathcal{G}\|_{H^{1+\ell}}\right) \leq C\left(C_{1}\right) \varepsilon^{2} \lambda^{2},
\end{aligned}
$$

where we have used the estimates in Propositions [7.2, 7.3 and 7.5 as well as bounds for the background solution in Corollary 3.5 .

Finally, we conclude this subsection with the following estimate on $\Delta\left(\mathfrak{g}-\mathfrak{g}_{0}-\mathfrak{g}_{1}-\mathfrak{g}_{2}\right)$ :

\section{Proposition 7.9.}

$$
\sum_{k \leq 3} \lambda^{k}\left\|\Delta\left(\mathfrak{g}-\mathfrak{g}_{0}-\mathfrak{g}_{1}-\mathfrak{g}_{2}\right)\right\|_{H_{\delta+2}^{k}}+\sum_{k \leq 2} \lambda^{k+1}\left\|\partial_{t}\left(\Delta\left(\mathfrak{g}-\mathfrak{g}_{0}-\mathfrak{g}_{1}-\mathfrak{g}_{2}\right)\right)\right\|_{H_{\delta+2}^{k}} \leq C\left(C_{1}\right) \varepsilon^{2} \lambda^{2} .
$$

Proof. We will only prove the first estimate as the second estimate is similar. By (4.35) and (4.36), we have

$$
\begin{aligned}
\Delta\left(\mathfrak{g}-\mathfrak{g}_{0}\right)= & \left(\boldsymbol{\Gamma}(\mathfrak{g})^{\mu \nu}-\boldsymbol{\Gamma}_{0}(\mathfrak{g})^{\mu \nu}\right) \partial_{\mu} \phi \partial_{\nu} \phi+\boldsymbol{\Gamma}_{0}(\mathfrak{g})^{\mu \nu}\left(\partial_{\mu} \phi \partial_{\nu} \phi-\partial_{\mu} \phi_{0} \partial_{\nu} \phi_{0}\right) \\
& -\frac{1}{2} \sum_{\mathbf{A}} F_{\mathbf{A}}^{2} \boldsymbol{\Gamma}_{0}(\mathfrak{g})^{\mu \nu}\left(\partial_{\mu} u_{\mathbf{A}}\right)\left(\partial_{\nu} u_{\mathbf{A}}\right)+\Upsilon(\mathfrak{g})-\Upsilon_{0}(\mathfrak{g}) .
\end{aligned}
$$


Using Proposition 7.1, this implies

$$
\Delta\left(\mathfrak{g}-\mathfrak{g}_{0}\right)=\left(\boldsymbol{\Gamma}(\mathfrak{g})^{\mu \nu}-\boldsymbol{\Gamma}_{0}(\mathfrak{g})^{\mu \nu}\right) \partial_{\mu} \phi \partial_{\nu} \phi+\boldsymbol{\Gamma}_{0}(\mathfrak{g})^{\mu \nu}\left(\Theta_{\mu \nu}^{(1)}+\Theta_{\mu \nu}^{(2)}+\Theta_{\mu \nu}^{(\text {remainder })}\right)+\Upsilon(\mathfrak{g})-\Upsilon_{0}(\mathfrak{g}) .
$$

By Corollary 3.5, (7.5) and Proposition $7.4, \boldsymbol{\Gamma}_{0}(\mathfrak{g})^{\mu \nu} \Theta_{\mu \nu}^{(\text {remainder })}$ and $\left(\boldsymbol{\Gamma}(\mathfrak{g})^{\mu \nu}-\boldsymbol{\Gamma}_{0}(\mathfrak{g})^{\mu \nu}\right) \partial_{\mu} \phi \partial_{\nu} \phi$ satisfy the desired estimate. As usual, we denote such terms by ... By Proposition 7.8 , we then have

$$
\Delta\left(\mathfrak{g}-\mathfrak{g}_{0}-\mathfrak{g}_{1}\right)=\Upsilon(\mathfrak{g})-\Upsilon_{0}(\mathfrak{g})+\Delta \mathfrak{g}_{2}-\Upsilon_{1}(\mathfrak{g})+\ldots
$$

We conclude thanks to Proposition 7.6

7.3. Estimates for $\mathfrak{g}_{1}$ and $\mathfrak{g}_{2}$. Recall the definition of $\mathfrak{g}_{1}$ in (4.37). By inspection and using the regularity of the background solution, we immediately have the following bounds, as is stated in (B2).

Proposition 7.10. $\mathfrak{g}_{1}$ depends only on the background solution, is compactly supported in $B\left(0, R_{\text {supp }}\right)$ and satisfies the estimate

$$
\sum_{k \leq 8} \lambda^{k}\left\|\mathfrak{g}_{1}\right\|_{H^{k} \cap C^{k}}+\sum_{k \leq 7} \lambda^{k+1}\left\|\partial_{t} \mathfrak{g}_{1}\right\|_{H^{k} \cap C^{k}}+\sum_{k \leq 6} \lambda^{k+2}\left\|\partial_{t}^{2} \mathfrak{g}_{1}\right\|_{H^{k} \cap C^{k}} \leq C\left(C_{0}\right) \varepsilon^{2} \lambda^{2} .
$$

For $\mathfrak{g}_{2}$, we again have by inspection that

Proposition 7.11. $\mathfrak{g}_{2}$ satisfies the following estimate:

$$
\sum_{k \leq 5} \lambda^{k}\left\|\mathfrak{g}_{2}\right\|_{H^{k}}+\sum_{k \leq 4} \lambda^{k+1}\left\|\partial_{t} \mathfrak{g}_{2}\right\|_{H^{k}} \leq C\left(C_{1}\right) \varepsilon^{2} \lambda^{3} .
$$

Proof. Using the definition (4.38) we have

$$
\lambda^{k}\left\|\mathfrak{g}_{2}\right\|_{H^{k}} \lesssim \lambda^{k} \sum_{\ell \leq k} \lambda^{3-k+\ell}\|\mathcal{G}\|_{H^{\ell}} \lesssim \varepsilon^{2} \lambda^{3}
$$

where we have used $\mathcal{G}$ to denote any of $\mathcal{G}^{\Delta}$, estimated thanks to Proposition $7.2 \mathcal{G}^{\Theta}$, estimated by Proposition 7.3 and $\mathcal{G}^{\Upsilon}$, estimated by Proposition 7.5. Notice that the regularity in this estimate is limited by that of $\widetilde{F}_{\mathbf{A}}$. The estimate for $\partial_{t} \mathfrak{g}_{2}$ is similar.

We note in particular that the estimate in Proposition 7.11 improves the bootstrap assumption (BA3).

7.4. Estimates for the term $\mathfrak{g}_{3}$. Next, we obtain the estimates for $\mathfrak{g}_{3}$. By Proposition [7.9, we already have a good estimate for $\Delta \mathfrak{g}_{3}$ and $\Delta\left(\partial_{t} \mathfrak{g}_{3}\right)$. Therefore, the following is a consequence of Corollary A.10.

Corollary 7.12.

$$
\mathfrak{g}_{3}=\left(\mathfrak{g}_{3}\right)_{\text {asymp }}(t) \chi(|x|) \log (|x|)+\widetilde{\mathfrak{g}}_{3},
$$

where

$$
\left|\left(\mathfrak{g}_{3}\right)_{\text {asymp }}\right|+\left|\partial_{t}\left(\mathfrak{g}_{3}\right)_{\text {asymp }}\right|+\sum_{k \leq 3} \lambda^{k}\left\|\widetilde{\mathfrak{g}}_{3}\right\|_{H_{\delta}^{2+k}}+\sum_{k \leq 2} \lambda^{k+1}\left\|\partial_{t} \widetilde{\mathfrak{g}}_{3}\right\|_{H_{\delta}^{2+k}} \leq C\left(C_{1}\right) \varepsilon^{2} \lambda^{2} .
$$

Remark 7.13. We have $\left(\beta_{3}\right)_{\text {asymp }}=\partial_{t}\left(\beta_{3}\right)_{\text {asymp }}=0$, as a consequence of the local well-posedness result (Theorem [3.3).

Notice that Corollary 7.12 is an improvement of (BA4).

7.5. Improved estimates for $\partial_{t} \gamma_{3}$. The key is the following lemma:

Lemma 7.14.

$$
\sum_{k \leq 7} \lambda^{k}\left\|\left(\partial_{t}-\beta_{0}^{i} \partial_{i}\right) \gamma_{1}-\frac{1}{2} \partial_{i} \beta_{1}^{i}\right\|_{H^{k} \cap C^{k}} \leq C\left(C_{0}\right) \varepsilon^{2} \lambda^{2} .
$$

Proof. This is proven by an explicit computation. For the purpose of this proof, it is helpful to introduce a notation for the background $e_{0}$ - we will denote $\mathbf{e}_{0}:=\partial_{t}-\beta_{0}^{i} \partial_{i}$. By (4.28) and (4.37), we have

$$
\begin{aligned}
\gamma_{1}= & \frac{1}{8} \sum_{\mathbf{A}} \frac{\lambda^{2} F_{\mathbf{A}}^{2}}{\left|\nabla u_{\mathbf{A}}\right|^{2}}\left(\left|\nabla u_{\mathbf{A}}\right|^{2}+\frac{e^{2 \gamma_{0}}}{N_{0}^{2}}\left(\mathbf{e}_{0} u_{\mathbf{A}}\right)\left(\mathbf{e}_{0} u_{\mathbf{A}}\right)\right) \cos \left(\frac{2 u_{\mathbf{A}}}{\lambda}\right) \\
& +2 \sum_{\mathbf{A}} \frac{\lambda^{2} F_{\mathbf{A}}}{\left|\nabla u_{\mathbf{A}}\right|^{2}}\left(\left(\nabla \phi_{0}\right) \cdot\left(\nabla u_{\mathbf{A}}\right)+\frac{e^{2 \gamma_{0}}}{N_{0}^{2}}\left(\mathbf{e}_{0} \phi_{0}\right)\left(\mathbf{e}_{0} u_{\mathbf{A}}\right)\right) \sin \left(\frac{u_{\mathbf{A}}}{\lambda}\right) \\
& +\frac{1}{2} \sum_{ \pm} \sum_{\mathbf{A}} \sum_{\mathbf{B} \neq \mathbf{A}} \frac{(\mp 1) \cdot \lambda^{2} F_{\mathbf{A}} F_{\mathbf{B}}}{\left|\nabla\left(u_{\mathbf{A}} \pm u_{\mathbf{B}}\right)\right|^{2}}\left(\left(\nabla u_{\mathbf{A}}\right) \cdot\left(\nabla u_{\mathbf{B}}\right)+\frac{e^{2 \gamma_{0}}}{N_{0}^{2}}\left(\mathbf{e}_{0} u_{\mathbf{A}}\right)\left(\mathbf{e}_{0} u_{\mathbf{B}}\right)\right) \cos \left(\frac{u_{\mathbf{A}} \pm u_{\mathbf{B}}}{\lambda}\right) .
\end{aligned}
$$


Differentiating, we obtain

$$
\begin{aligned}
\mathbf{e}_{0} \gamma_{1}= & -\frac{1}{4} \sum_{\mathbf{A}} \frac{\lambda F_{\mathbf{A}}^{2}}{\left|\nabla u_{\mathbf{A}}\right|^{2}}\left(\mathbf{e}_{0} u_{\mathbf{A}}\right)\left(\left|\nabla u_{\mathbf{A}}\right|^{2}+\frac{e^{2 \gamma_{0}}}{N_{0}^{2}}\left(\mathbf{e}_{0} u_{\mathbf{A}}\right)^{2}\right) \sin \left(\frac{2 u_{\mathbf{A}}}{\lambda}\right) \\
& +2 \sum_{\mathbf{A}} \frac{\lambda F_{\mathbf{A}}}{\left|\nabla u_{\mathbf{A}}\right|^{2}}\left(\mathbf{e}_{0} u_{\mathbf{A}}\right)\left(\left(\nabla \phi_{0}\right) \cdot\left(\nabla u_{\mathbf{A}}\right)+\frac{e^{2 \gamma_{0}}}{N_{0}^{2}}\left(\mathbf{e}_{0} \phi_{0}\right)\left(\mathbf{e}_{0} u_{\mathbf{A}}\right)\right) \cos \left(\frac{u_{\mathbf{A}}}{\lambda}\right) \\
& -\frac{1}{2} \sum_{ \pm} \sum_{\mathbf{A}} \sum_{\mathbf{B} \neq \mathbf{A}} \frac{(\mp 1) \cdot \lambda F_{\mathbf{A}} F_{\mathbf{B}}}{\left|\nabla\left(u_{\mathbf{A}} \pm u_{\mathbf{B}}\right)\right|^{2}}\left(\mathbf{e}_{0}\left(u_{\mathbf{A}} \pm u_{\mathbf{B}}\right)\right) \\
& \times\left(\left(\nabla u_{\mathbf{A}}\right) \cdot\left(\nabla u_{\mathbf{B}}\right)+\frac{e^{2 \gamma_{0}}}{N_{0}^{2}}\left(\mathbf{e}_{0} u_{\mathbf{A}}\right)\left(\mathbf{e}_{0} u_{\mathbf{B}}\right)\right) \sin \left(\frac{u_{\mathbf{A}} \pm u_{\mathbf{B}}}{\lambda}\right)+\ldots,
\end{aligned}
$$

where here, and below in this proof, we have used ... to denote terms with $\sum_{k \leq 3} \lambda^{k}\|\cdot\|_{H^{k} \cap C^{k}}$ norms bounded above by $C\left(C_{0}\right) \varepsilon^{2} \lambda^{2}$. (These terms arise when the derivative does not act on the oscillating factors.) On the other hand, we compute $\beta_{1}^{i}$ according to (4.30), (4.31) and (4.37) to get

$$
\begin{aligned}
\beta_{1}^{i}= & \frac{1}{2} \delta^{i j} \sum_{\mathbf{A}} \frac{\lambda^{2} F_{\mathbf{A}}^{2}}{\left|\nabla u_{\mathbf{A}}\right|^{2}}\left(\mathbf{e}_{0} u_{\mathbf{A}}\right)\left(\partial_{j} u_{\mathbf{A}}\right) \cos \left(\frac{2 u_{\mathbf{A}}}{\lambda}\right) \\
& +4 \delta^{i j} \sum_{\mathbf{A}} \frac{\lambda^{2} F_{\mathbf{A}}}{\left|\nabla u_{\mathbf{A}}\right|^{2}}\left(\left(\mathbf{e}_{0} \phi_{0}\right)\left(\partial_{j} u_{\mathbf{A}}\right)+\left(\mathbf{e}_{0} u_{\mathbf{A}}\right)\left(\partial_{j} \phi_{0}\right)\right) \sin \left(\frac{u_{\mathbf{A}}}{\lambda}\right) \\
& +2 \delta^{i j} \sum_{ \pm} \sum_{\mathbf{A}} \sum_{\mathbf{B} \neq \mathbf{A}} \frac{(\mp 1) \cdot \lambda^{2} F_{\mathbf{A}} F_{\mathbf{B}}}{\left|\nabla\left(u_{\mathbf{A}} \pm u_{\mathbf{B}}\right)\right|^{2}}\left(\mathbf{e}_{0} u_{\mathbf{A}}\right)\left(\partial_{j} u_{\mathbf{B}}\right) \cos \left(\frac{u_{\mathbf{A}} \pm u_{\mathbf{B}}}{\lambda}\right) .
\end{aligned}
$$

Therefore, taking the divergence (with respect to $\delta_{i j}$ ), (and noting that the last term remains unchanged under $\mathbf{A} \leftrightarrow \mathbf{B}$ ), we obtain

$$
\begin{aligned}
\partial_{i} \beta_{1}^{i}= & -\sum_{\mathbf{A}} \frac{\lambda F_{\mathbf{A}}^{2}}{\left|\nabla u_{\mathbf{A}}\right|^{2}}\left(\mathbf{e}_{0} u_{\mathbf{A}}\right)\left|\nabla u_{\mathbf{A}}\right|^{2} \sin \left(\frac{2 u_{\mathbf{A}}}{\lambda}\right) \\
& +4 \sum_{\mathbf{A}} \frac{\lambda F_{\mathbf{A}}}{\left|\nabla u_{\mathbf{A}}\right|^{2}}\left(\left(\mathbf{e}_{0} \phi_{0}\right)\left|\nabla u_{\mathbf{A}}\right|^{2}+\left(\mathbf{e}_{0} u_{\mathbf{A}}\right)\left(\left(\nabla \phi_{0}\right) \cdot\left(\nabla u_{\mathbf{A}}\right)\right)\right) \cos \left(\frac{u_{\mathbf{A}}}{\lambda}\right) \\
& -\sum_{ \pm} \sum_{\mathbf{A}} \sum_{\mathbf{B} \neq \mathbf{A}} \frac{(\mp 1) \cdot \lambda F_{\mathbf{A}} F_{\mathbf{B}}}{\left|\nabla\left(u_{\mathbf{A}} \pm u_{\mathbf{B}}\right)\right|^{2}}\left(\mathbf{e}_{0} u_{\mathbf{A}}\right)\left(\left(\nabla u_{\mathbf{B}}\right) \cdot\left(\nabla\left(u_{\mathbf{A}} \pm u_{\mathbf{B}}\right)\right) \sin \left(\frac{u_{\mathbf{A}} \pm u_{\mathbf{B}}}{\lambda}\right)\right. \\
& -\sum_{ \pm} \sum_{\mathbf{A}} \sum_{\mathbf{B} \neq \mathbf{A}} \frac{(\mp 1) \cdot \lambda F_{\mathbf{A}} F_{\mathbf{B}}}{\left|\nabla\left(u_{\mathbf{A}} \pm u_{\mathbf{B}}\right)\right|^{2}}\left(\mathbf{e}_{0} u_{\mathbf{B}}\right)\left(\left(\nabla u_{\mathbf{A}}\right) \cdot\left(\nabla\left(u_{\mathbf{A}} \pm u_{\mathbf{B}}\right)\right) \sin \left(\frac{u_{\mathbf{A}} \pm u_{\mathbf{B}}}{\lambda}\right)+\ldots\right.
\end{aligned}
$$

Finally, recalling that $u_{\mathbf{A}}$ is an eikonal function on the background, we have $\frac{e^{2 \gamma_{0}}}{N_{0}^{2}}\left(\mathbf{e}_{0} u_{\mathbf{A}}\right)^{2}=\left|\nabla u_{\mathbf{A}}\right|^{2}$. Therefore, in the expression $\left(\mathbf{e}_{0} \gamma_{1}-\frac{1}{2} \partial_{i} \beta_{1}^{i}\right)$, the main contributions in (7.20) and (7.21) cancel exactly. This yields the proposition.

Equipped with Lemma 7.14, the necessary improved estimates for $\partial_{t} \gamma_{3}$ follows easily from the gauge conditions:

\section{Proposition 7.15 .}

$$
\left\|\partial_{t} \gamma_{3}\right\|_{L^{2}\left(B\left(0, R_{\text {supp }}+1\right)\right)} \leq C\left(C_{1}\right) \varepsilon^{2} \lambda^{2} .
$$

Proof. Since the mean curvature of each $\Sigma_{t}$ vanishes, we have

$$
\left(\partial_{t}-\beta^{i} \partial_{i}\right) \gamma=\frac{1}{2} \partial_{i} \beta^{i}
$$

The same formula holds for the background, with $\gamma \mapsto \gamma_{0}$ and $\beta^{i} \mapsto \beta_{0}^{i}$, i.e.,

$$
\left(\partial_{t}-\beta_{0}^{i} \partial_{i}\right) \gamma_{0}=\frac{1}{2} \partial_{i} \beta_{0}^{i} \text {. }
$$

Subtracting, we thus obtain

$$
\left(\partial_{t}-\beta_{0}^{i} \partial_{i}\right)\left(\gamma-\gamma_{0}\right)=\frac{1}{2} \partial_{i}\left(\beta^{i}-\beta_{0}^{i}\right)+\left(\beta^{i}-\beta_{0}^{i}\right) \partial_{i} \gamma
$$



i.e.,

As a consequence,

$$
\left(\partial_{t}-\beta_{0}^{i} \partial_{i}\right)\left(\gamma_{1}+\gamma_{2}+\gamma_{3}\right)=\frac{1}{2} \partial_{i}\left(\beta_{1}^{i}+\beta_{2}^{i}+\beta_{3}^{i}\right)+\left(\beta^{i}-\beta_{0}^{i}\right) \partial_{i} \gamma
$$

$$
\begin{aligned}
\left\|\partial_{t} \gamma_{3}\right\|_{L^{2}\left(B\left(0, R_{\text {supp }}+1\right)\right)} & \\
\leq C\left(C_{0}\right) & \left(\left\|\partial \mathfrak{g}_{2}\right\|_{L^{2}\left(B\left(0, R_{\text {supp }}+1\right)\right)}+\left\|\nabla \mathfrak{g}_{3}\right\|_{L^{2}\left(B\left(0, R_{\text {supp }}+1\right)\right)}+\left\|\left(\partial_{t}-\beta_{0}^{i} \partial_{i}\right) \gamma_{1}-\frac{1}{2} \partial_{i} \beta_{1}^{i}\right\|_{L^{2}\left(B\left(0, R_{\text {supp }}+1\right)\right)}\right. \\
& \left.+\left\|\beta^{i}-\beta_{0}^{i}\right\|_{L^{\infty}\left(B\left(0, R_{\text {supp }}+1\right)\right.}\left\|\partial_{i} \gamma\right\|_{L^{2}\left(B\left(0, R_{\text {supp }}+1\right)\right.}\right) \leq C\left(C_{1}\right) \varepsilon^{2} \lambda^{2},
\end{aligned}
$$

where we have used (B2), (BA3), (BA4), (5.7), (5.8), as well as Lemma 7.14 .

Since on the compact set $B\left(0, R_{\text {supp }}+1\right), \partial_{t} \gamma_{3}=\partial_{t} \widetilde{\gamma}_{3}$, we have thus improved the bootstrap assumption (BA5).

\section{Putting everything together: Proof of Theorem 4.2}

Thanks to Propositions 6.15, Corollary 6.23, Proposition 7.11, Corollary 7.12 and Proposition 7.15 we have proved the estimates in the bootstrap assumptions (BA1)-(BA5), with $C_{1} \varepsilon$ replaced by $C\left(C_{0}\right) \varepsilon+$ $C\left(C_{1}\right) \varepsilon^{2}$, where $C\left(C_{0}\right)$ is a constant, depending on the background and independent of $C_{1}$. We can choose $C_{1}$ sufficiently large such that $C_{1} \geq 4 C\left(C_{0}\right)$, and $\varepsilon$ sufficiently small such that $C\left(C_{1}\right) \varepsilon \leq \frac{1}{4} C_{1}$, we then have improved the estimates in in the bootstrap assumptions (BA1)-(BA5), with $C_{1}$ replaced by $\frac{C_{1}}{2}$. This proves the bootstrap theorem (Theorem [5.2).

Now, by the the local existence theorem (Theorem 3.3), we see that when $F_{\mathbf{A}} \equiv 0$ (which is the case under consideration), the solution only breaks down when at least one of the following holds:

(1) (Higher norms of matter fields blow up)

$$
\liminf _{t \rightarrow T_{*}}\left(\left\|\frac{e^{2 \gamma}}{N}\left(e_{0} \phi\right)\right\|_{H^{k}}(t)+\|\nabla \phi\|_{H^{k}}(t)+\max _{\mathbf{A}}\left\|F_{\mathbf{A}} e^{\frac{\gamma}{2}}\right\|_{H^{k}}(t)\right)=+\infty
$$

(2) (Lower norms of matter fields leave smallness regime 34 )

$$
\liminf _{t \rightarrow T_{*}}\left(\left\|\frac{e^{2 \gamma}}{N}\left(e_{0} \phi\right)\right\|_{L^{\infty}}(t)+\|\nabla \phi\|_{L^{\infty}}(t)+\max _{\mathbf{A}}\left\|F_{\mathbf{A}} e^{\frac{\gamma}{2}}\right\|_{L^{\infty}}(t)\right)>\varepsilon_{\text {low }} .
$$

However, by choosing $\varepsilon$ sufficiently small, the estimates in the bootstrap argument precisely show that neither of these can occur. Hence, the solution exists on the whole time interval $[0,1]$.

It remains to show that we have the desired convergence, which follows easily from the parametrix (4.18), (4.24), and estimates established in the bootstrap argument. This concludes the proof of Theorem 4.2

\section{Appendix A. Weighted Sobolev spaces}

For the sake of completeness, we recall here useful properties on weighted Sobolev spaces. For relevant definitions, see Definition 2.1. Unless otherwise stated, we will only be interested in weighted Sobolev spaces on $\mathbb{R}^{2}$. Most of the results can be found in [3, Appendix I] (although we use slightly different notations).

A.1. Embedding theorems. The following lemma is an immediate consequence of the definition.

Lemma A.1. Let $m \geq 1, p \in[1, \infty)$ and $\delta \in \mathbb{R}$. Then for $j=1,2$,

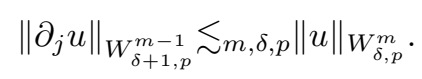

Similarly, for $m \geq 1, \delta \in \mathbb{R}, j=1,2$,

$$
\left\|\partial_{j} u\right\|_{C_{\delta+1}^{m-1}} \lesssim m, \delta, p\|u\|_{C_{\delta}^{m}}
$$

We have an easy embedding result, which is a straightforward application of the Hölder's inequality:

Lemma A.2. If $1 \leq p_{1} \leq p_{2} \leq \infty$ and $\delta_{2}-\delta_{1}>2\left(\frac{1}{p_{1}}-\frac{1}{p_{2}}\right)$, then we have the continuous embedding

$$
W_{\delta_{2}, p_{2}}^{0} \subset W_{\delta_{1}, p_{1}}^{0} \text {. }
$$

\footnotetext{
${ }^{34}$ Note that with our estimates, we also have control over the support of the solution. Therefore, according to Theorem 3.3 there is indeed an $\varepsilon_{\text {low }}$ that we can talk about, which depends on this upper bound of the support of the solution, as well as $k$ and $\delta$.
} 
The following simple lemma will be useful as well.

Lemma A.3. Let $\alpha \in \mathbb{R}$ and $g \in L_{\text {loc }}^{\infty}$ be such that

$$
|g(x)| \lesssim\left(1+|x|^{2}\right)^{\alpha} .
$$

Then the multiplication by $g$ maps $H_{\delta}^{0}$ to $H_{\delta-2 \alpha}^{0}$ with operator norm bounded by $\sup _{x \in \mathbb{R}^{2}} \frac{|g(x)|}{\left(1+|x|^{2}\right)^{\alpha}}$.

Next, we have a Sobolev embedding theorem with weights:

Proposition A.4. Let $s, m \in \mathbb{N} \cup\{0\}, 1<p<\infty$. The following Sobolev embedding theorems hold:

- Suppose $s>\frac{2}{p}$ and $\beta \leq \delta+\frac{2}{p}$. Then, we have the continuous embedding

$$
W_{\delta, p}^{s+m} \subset C_{\beta}^{m} .
$$

- Suppose $s<\frac{2}{p}$. Then, we have the continuous embedding

$$
W_{\delta, p}^{s+m} \subset W_{\delta+s, \frac{n p}{n-s p}}^{m} .
$$

- Suppose $s=\frac{2}{p}$. Then, we have the continuous embedding for all $q<\infty$

$$
W_{\delta, p}^{s+m} \subset W_{\delta+s, q}^{m} .
$$

It will be convenient to prove a refined (in terms of scaling) Sobolev embedding theorem for the $H^{2} \subset L^{\infty}$ embedding:

Proposition A.5. The following holds for all functions $u$ such that the RHS is finite:

$$
\|u\|_{L^{\infty}} \lesssim\|u\|_{L^{2}}^{\frac{1}{2}}\left(\sum_{|\alpha|=2}\left\|\nabla^{\alpha} u\right\|_{L^{2}}\right)^{\frac{1}{2}} .
$$

Proof. By Proposition A.4 for every $v$,

$$
\|v\|_{L^{\infty}} \lesssim\|v\|_{L^{2}}+\sum_{|\alpha|=2}\left\|\nabla^{\alpha} v\right\|_{L^{2}} .
$$

Apply this to $v(x)=u_{\mu}(x):=u\left(\frac{x}{\mu}\right)$ for $\mu>0$, we obtain

$$
\|u\|_{L^{\infty}} \lesssim \mu\|u\|_{L^{2}}+\mu^{-1} \sum_{|\alpha|=2}\left\|\nabla^{\alpha} u\right\|_{L^{2}}
$$

Choose $45=\|u\|_{L^{2}}^{-\frac{1}{2}}\left(\sum_{|\alpha|=2}\left\|\nabla^{\alpha} u\right\|_{L^{2}}\right)^{\frac{1}{2}}$ yields the conclusion.

A.2. Product estimates. We have three product estimates. The first can be found in [3] Appendix I].

Proposition A.6. Let $s, s_{1}, s_{2} \in \mathbb{N} \cup\{0\}, p \in[1, \infty], \delta, \delta_{1}, \delta_{2} \in \mathbb{R}$. Assume that $s \leq \min \left(s_{1}, s_{2}\right)$ and $s<s_{1}+s_{2}-\frac{2}{p}$. Let $\delta<\delta_{1}+\delta_{2}+\frac{2}{p}$. Then $\forall(u, v) \in W_{\delta_{1}, p}^{s_{1}} \times W_{\delta_{2}, p}^{s_{2}}$,

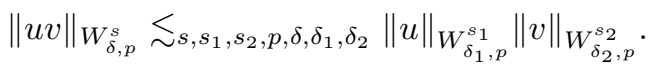

The second is for unweighted Sobolev spaces, which can be found in [16, Appendix A].

Proposition A.7. Let $s \in \mathbb{N}$. Then $\forall(u, v) \in\left(H^{s} \cap L^{\infty}\right) \times\left(H^{s} \cap L^{\infty}\right)$,

$$
\|u v\|_{H^{s}} \lesssim s\|u\|_{H^{s}}\|v\|_{L^{\infty}}+\|u\|_{L^{\infty}}\|v\|_{H^{s}}
$$

The third product estimate is an immediate corollary of Proposition A.7, which is useful when one of the two functions is compactly supported.

Proposition A.8. Let $s \in \mathbb{N}$. Then $\forall(u, v) \in\left(H^{s} \cap L^{\infty}\right) \times\left(H^{s} \cap L^{\infty}\right)$ such that $\operatorname{supp}(u) \subset B\left(0, R_{\text {supp }}\right)$,

$$
\|u v\|_{H^{s}} \lesssim_{s, R_{\text {supp }}}\|u\|_{H^{s}}\|v\|_{L^{\infty}\left(B\left(0, R_{\text {supp }}+1\right)\right)}+\|u\|_{L^{\infty}}\|v\|_{H^{s}\left(B\left(0, R_{\text {supp }}+1\right)\right)} .
$$

Proof. Let $\eta$ be a cutoff function compactly supported in $B\left(0, R_{\text {supp }}+1\right)$ which is $\equiv 1$ in $B\left(0, R_{\text {supp }}\right)$. Noting that $u v=u v \eta$, we apply Proposition A.7 with $(u, v \eta)$ instead of $(u, v)$.

\footnotetext{
${ }^{35}$ We can assume without loss of generality that $\|u\|_{L^{2}} \neq 0$ for otherwise the conclusion is trivial.
} 
A.3. Inversion of the Laplacian. We then discuss the invertibility of the Laplacian on weighted Sobolev spaces. The following theorem is due to McOwen:

Theorem A.9. (Theorem 0 in [15]) Let $m \in \mathbb{Z}, m \geq 0,1<p<\infty$ and $-\frac{2}{p}+m<\delta<m+1-\frac{2}{p}$. The Laplace operator $\Delta: W_{\delta, p}^{2+m} \rightarrow W_{\delta+2, p}^{m}$ is an injection with closed range

$$
\left\{f \in W_{\delta+2, p}^{m} \mid \int f v=0 \quad \forall v \in \cup_{i=0}^{m} \mathcal{H}_{i}\right\},
$$

where $\mathcal{H}_{i}$ is the set of harmonic polynomials of degree $i$. Moreover, $u$ obeys the estimate

$$
\|u\|_{W_{\delta, p}^{2+m}} \leq C(\delta, m, p)\|\Delta u\|_{W_{\delta+2, p}^{m}}
$$

where $C(\delta, m, p)>0$ is a constant depending on $\delta, m$ and $p$.

An immediate corollary is the following:

Corollary A.10. Let $-1<\delta<0$ and $f \in H_{\delta+2}^{0}$. Then there exists a solution $u$ of

$$
\Delta u=f
$$

which can be written

$$
u=\frac{1}{2 \pi}\left(\int f\right) \chi(|x|) \log (|x|)+v
$$

where $\chi:[0, \infty) \rightarrow \mathbb{R}$ is a smooth cutoff function $=0$ for $|x| \leq 1$ and $=1$ for $|x| \geq 2$, and $v \in H_{\delta}^{2}$ is such that $\|v\|_{H_{\delta}^{2}} \leq C(\delta)\|f\|_{H_{\delta+2}^{0}}$.

\section{REFERENCES}

[1] Gregory A. Burnett, The high-frequency limit in general relativity, J. of Math. Phys. 30, 90-96 (1989).

[2] Yvonne Choquet-Bruhat, Construction de solutions radiatives approchées des équations d'Einstein, Comm. Math. Phys. 12, 16-35 (1969).

[3] Yvonne Choquet-Bruhat, General Relativity and the Einstein Equations, Oxford Mathematical Monographs (2009).

[4] Stephen R. Green and Robert M. Wald, New framework for analyzing the effects of small scale inhomogeneities in cosmology, Phys. Rev. D 83, 084020, arXiv:1011.4920 (2011).

[5] Stephen R. Green and Robert M. Wald, Examples of backreaction of small scale inhomogeneities in cosmology, Phys. Rev. D 87, 124037, arXiv:1304.2318 (2013).

[6] Cécile Huneau, Constraint equations for $3+1$ vacuum Einstein equations with a translational space-like Killing field in the asymptotically flat case, Ann. Henri Poincaré 17(2), 271-299, arXiv:1302.1473 (2016).

[7] Cécile Huneau and Jonathan Luk, Einstein equations under polarized U(1) symmetry in an elliptic gauge, preprint (2017).

[8] Richard A. Isaacson, Gravitational Radiation in the Limit of High Frequency. I. The Linear Approximation and Geometrical Optics, Phys. Rev. 166, 1263-1271 (1968).

[9] Richard A. Isaacson, Gravitational Radiation in the Limit of High Frequency. II. Nonlinear Terms and the Effective Stress Tensor, Phys. Rev. 166, 1272-1279 (1968).

[10] Sergiu Klainerman, Igor Rodnianski and Jérémie Szeftel, The bounded L2 curvature conjecture, Invent. Math., 202(1), 91-216, arXiv:1204.1767 (2015).

[11] Jonathan Luk and Igor Rodnianski, Local propagation of impulsive gravitational waves, Comm. Pure and Appl. Math. 68(4), 511-624, arXiv:1209.1130 (2015).

[12] Jonathan Luk and Igor Rodnianski, Nonlinear interaction of impulsive gravitational waves for the vacuum Einstein equations, to appear in Cambridge Journal of Math., arXiv:1301.1072 (2013).

[13] Jonathan Luk and Igor Rodnianski, High frequency limits in general relativity, in preparation.

[14] M. A. H. MacCallum and A. H. Taub, The averaged Lagrangian and high-frequency gravitational waves, Comm. Math. Phys. 30(2), 153-169 (1973).

[15] Robert C. McOwen, The behavior of the Laplacian on weighted Sobolev spaces, Comm. Pure Appl. Math. 32(6), 783-795 (1979).

[16] Terence Tao, Nonlinear Dispersive Equations: Local and Global Analysis, CBMS Regional Conference Series in Mathematics Volume: 106, 2006.

Institut Fourier, Université Grenoble-Alpes, 100 Rue des maths, 38610 Gières, FranCe

E-mail address: cecile.huneau@univ-grenoble-alpes.fr

Department of Mathematics, Stanford University, CA 94304, USA

E-mail address: jluk@stanford.edu 